\title{
DRONE CHARACTERIZATION USING HELICOPTER ROTATION MODULATION (HERM) LINE SIGNATURES
}

\author{
By \\ Andi Huang

\begin{abstract}
A thesis
submitted to the Faculty of Graduate and Postdoctoral Affairs

in partial fulfillment of the requirements

for the degree of

Master of Applied Science

in
\end{abstract}

Electrical and Computer Engineering

Carleton University

Ottawa, Ontario

(C) Copyright 2021 


\section{Abstract}

This thesis presents research on the radar micro-Doppler drone characterization using features from the long windowed short-time Fourier transform (STFT) representation referred to as HElicopter Rotation Modulation (HERM) line signatures. The long windowed STFT representation enables drone identification at longer ranges using a low pulse repetition frequency $(\mathrm{PRF})$ radars. A simple mathematical model is proposed to describe the HERM line signature using three parameters or features, namely the number of HERM lines, the fundamental frequencies and the number of fundamental frequencies. This model motivates research into parametric spectral estimation techniques for micro-Doppler feature extraction which is lacking in the current state of the art. Subspace-based spectral estimation techniques are applied to real and simulated data and compared with the Fourier-based techniques from the literature. These proposed techniques are shown to perform better for smaller windows of data. An application of these features for drone detection/classification is demonstrated. 


\section{Acknowledgements}

I would like to thank my advisor Dr. Sreeraman Rajan for giving me this wonderful opportunity and giving me support and advice.

I would like to thank my supervisor Dr. Bhashyam Balaji for always supporting my ideas and providing the necessary equipment at Defence Research and Development Canada (DRDC).

I would like to thank Dr. Peter Klaer for helping me run experiments and collect data. I couldn't of done it without you.

I would like to thank my fellow researcher Pascale Sévigny for all her help during this project.

I would like to thank Hoissein Chahour for teaching me how to use the TI radar.

I want to thank Shashank Pant and everyone at National Research Council Canada (NRC) for making the drone trials possible. 


\section{Contents}

$\begin{array}{ll}\text { Abstract } & \text { ii }\end{array}$

Acknowledgements

List of Abbreviations $\quad$ xiii

1 Introduction 1

1.1 Motivation . . . . . . . . . . . . . . . . . . 1

1.2 Problem Statement . . . . . . . . . . . . . . . . . . . . 4

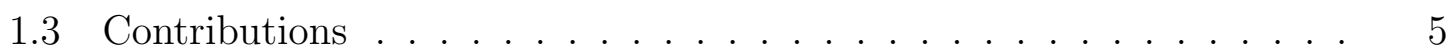

1.4 Limitations of the Thesis . . . . . . . . . . . . . 7

1.5 Thesis Overview . . . . . . . . . . . . . . . . . 8

2 Background $\quad 10$

2.1 Radar Basics . . . . . . . . . . . . . . . . . . . 10

2.2 Doppler Frequency . . . . . . . . . . . . . . . . . . . . . 12

2.3 Pulse Compression . . . . . . . . . . . . . . . . . . . . . 15

2.4 Frequency Modulated Continuous Wave Radars . . . . . . . . . 16

2.5 Rotor Micro-Doppler Effect . . . . . . . . . . . . . . . . 17

2.6 Micro-Doppler Signatures _ . . . . . . . . . . . . . . . . . . . . 18

2.6 .1 STFT Spectrogram . . . . . . . . . . . . . . . . 18

2.7 Literature on Drone Classification using Micro-Doppler . . . . . . . . 24

2.7.1 Short-window STFT Spectrogram Methods . . . . . . . . . 24

2.7.2 Modified Short-Window Spectrogram Representations . . . . 25 
2.7.3 Long-Window HERM line-based Methods . . . . . . . . 27

2.7.4 Non-Fourier Based Methods . . . . . . . . . . . . . . . 28

2.8 Limitations and Conclusion . . . . . . . . . . . . . . . . 30

3 Experimental Trials and Micro-Doppler Model 31

3.1 Data collection protocol . . . . . . . . . . . . . . . . 31

3.2 Experimental Results . . . . . . . . . . . . . . . . . . . . . 34

3.2 .1 Air Hogs Havoc Micro-helicopter . . . . . . . . . . . . . 34

3.2 .2 Multi-rotor Drones . . . . . . . . . . . . . . . . 36

3.2 .3 Parrot Disco Fixed Wing Drone . . . . . . . . . . . . 38

3.2 .4 Birds . . . . . . . . . . . . . . . . . . . . . . . . 39

3.3 Time Series Model of Rotor Micro-Doppler . . . . . . . . . . . . . 41

3.3.1 Time Series Model . . . . . . . . . . . . . . . . . . . . . . 41

3.3.2 Short-Window and Long-Window STFT . . . . . . . . . 43

3.3.3 Comparison with Martin-Mulgrew Models . . . . . . . . . . 44

3.4 Conclusions . . . . . . . . . . . . . . . . . . . 47

4 Parametric Estimation of the Number of HERM lines 49

4.1 Introduction . . . . . . . . . . . . . . . 50

4.2 Parametric Spectral Estimation Techniques . . . . . . . . . . . . 52

4.2.1 Minimum Description Length (MDL) \& Aikaike Information Criterion $(\mathrm{AIC}) \ldots \ldots \ldots \ldots \ldots$

4.2 .2 Radoi Method . . . . . . . . . . . . . . . . . . . . . 53

4.3 Performance Evaluation using Time Series Micro-Doppler Model . . . 54

4.3 .1 Single Rotor Drone . . . . . . . . . . . . . . . . . . . 54

4.3 .2 Multi-Rotor Drone . . . . . . . . . . . . . . . . 56

4.4 Experimental Results . . . . . . . . . . . . . . . . . . 58

4.4 .1 Single Rotor Drone . . . . . . . . . . . . . . . . . 58

4.4 .2 Multi-rotor drone . . . . . . . . . . . . . . . . . . 61

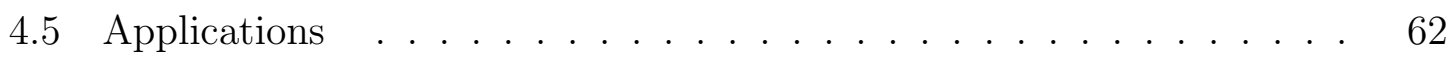

4.5.1 Rotary Drone Detector . . . . . . . . . . . . . . . . 62

4.6 Computational Complexity . . . . . . . . . . . . . . . 71 
4.7 Conclusion . . . . . . . . . . . . . . . . . . . . 71

5 Estimating HERM line Fundamental Frequency 73

5.1 Existing Methods . . . . . . . . . . . . . . . . . 74

5.1 .1 Cepstrum . . . . . . . . . . . . . . . . 74

5.1 .2 Cadence-Velocity Diagram . . . . . . . . . . . . . 75

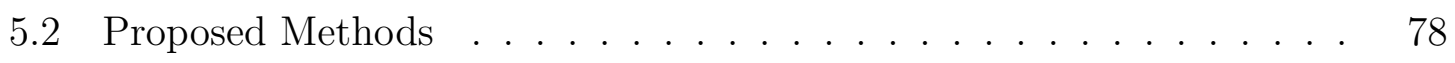

5.2 .1 Log Harmonic Summation . . . . . . . . . . . . . . . 78

5.2.2 Fundamental Frequency Estimation using MUSIC . . . . . . . 81

5.3 Performance Evaluation with Time Series Micro-Doppler Model . . . 84

5.4 Performance Evaluation with Measured Micro-Doppler signal . . . . 88

5.4.1 Short Observation Window . . . . . . . . . . . . . . . . 90

5.5 Drone Classification . . . . . . . . . . . . . . . . . . . . . . 93

5.6 Conclusion . . . . . . . . . . . . . . . . . . . . . . . . . . . . 97

6 Conclusion $\quad 99$

6.1 Limitations and Future Work . . . . . . . . . . . . . . . . 101

$\begin{array}{ll}\text { Bibliography } & 102\end{array}$

$\begin{array}{ll}\text { A FMCW radar theory } & 109\end{array}$

A.1 FMCW Radar . . . . . . . . . . . . . . . . . . . . . . . . . . . . 109

B Radars used in this thesis 116

B.1 Xethru X4 UWB radar . . . . . . . . . . . . . . . . . . . . . 116

B.2 TI AWR1642 FMCW Radar . . . . . . . . . . . . . . . . . . . . 118

B.3 Ancortek Radars . . . . . . . . . . . . . . . . . . . . . . . . . . . . 124

$\begin{array}{ll}\text { C Radar Performance Evaluation } & 126\end{array}$ 


\section{List of Tables}

1 Comparison of drone detection methods. . . . . . . . . . . . . 2

2 UAV specifications. . . . . . . . . . . . . . . . . . . . . 32

3 AWR1642 Radar Configuration. . . . . . . . . . . . . . . . 33

4 Ancortek Radar SDR-KIT 580 AD2 Configuration. . . . . . . . . 33

5 Xethru Radar Configuration. . . . . . . . . . . . . . . . . . . 33

6 HERM line separation frequency for different drones. . . . . . . . . . 47

7 Single Rotor HERM line model parameters. . . . . . . . . . . . 55

8 Multi-rotor HERM line model parameters. . . . . . . . . . . . . . 58

9 Estimated number of HERM lines using Radoi and Spectrogram Peak Detection Method. . . . . . . . . . . . . . . . . . . 60

10 Detection Performance for MDL for different segment lengths. . . . . 66

11 Detection Performance using MDL for different covariance matrix size

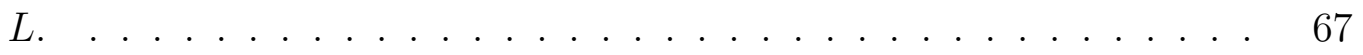

12 Single rotor HERM line model parameters. . . . . . . . . . . . . . 84

13 Effect of varying the number of harmonics summed up for the log harmonic summation method in the presence of $10 \mathrm{~dB}$ noise. . . . . 84

14 Comparison of fundamental frequency estimate algorithms. . . . . . 86

15 True fundamental frequency estimate rate. . . . . . . . . . . . . 91

16 Confusion matrix for Naive Bayes classifier using number of harmonic components as a feature. . . . . . . . . . . . . 96

17 Confusion matrix for Naive Bayes classifier using fundamental frequency as a feature. . . . . . . . . . . . . . 96

18 Confusion matrix for Naive Bayes classifier using both features. . . . 97 
19 Xethru X4 radar specifications. . . . . . . . . . . . . . . . . . 118

20 TI AWR1642 radar chirp parameters. . . . . . . . . . . . . . . 120

21 TI AWR1642 radar specifications. . . . . . . . . . . . . . 123

22 Ancortek radar specifications. . . . . . . . . . . . . . . 127

23 Input SNR Calculation. . . . . . . . . . . . . . . 128 


\section{List of Figures}

1 Techniques for estimating number of HERM lines. . . . . . . . . . . . 8

2 Techniques for estimating the micro-Doppler fundamental frequency. . 9

3 Monostatic radar setup [12]. . . . . . . . . . . . . . . . 11

4 Single channel radar data matrix. . . . . . . . . . . . . . . . . . 14

5 Micro-Doppler spectrogram of a two bladed helicopter with a 6 inch diameter propeller and a rotation rate of 34 revolutions per second measured using the TI FMCW radar. . . . . . . . . . . . . . . . . . . 19

6 The minimum PRF required for unambiguous sampling of the Doppler shift of a $10 \mathrm{~cm}$ propeller spinning at $80 \mathrm{~Hz}$ as a function of carrier frequency. . . . . . . . . . . . . . . . . . 21

$7 \quad$ Spectrogram smearing due to small STFT window size of $16 \ldots$. . . . 22

8 HERM line spectrogram generated from data collected using X-band radar with a sampling frequency of $1.5 \mathrm{KHz}$. Left: Window size of 64 $(\approx 0.04 \mathrm{~s})$, Right: Window size of $512(\approx 0.33 \mathrm{~s}) . \ldots . . . . . . .23$

$9 \quad$ First 8 IMFs of a single rotor blade[29]. . . . . . . . . . . . . . . . . 29

10 a) Air Hogs Havoc micro-helicopter drone b) DJI Spark quadcopter c) Typhoon H hexacopter d) Parrot Disco fixed wing drone. . . . . . . . 32

11 Experimental setup for drone trials at NRC . . . . . . . . . . . . . 34

12 Micro-Doppler signature captured by the TI radar on the left and HERM line signature from the Xethru radar on the right. . . . . .

13 Zoomed in micro-Doppler spectrogram of micro-helicopter drone at steady state. 
14 HERM line signature of hexacopter on a stand with varying rotor

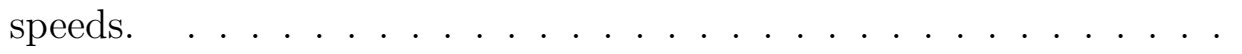

15 HERM line signature of hovering DJI Spark $4 \mathrm{~m}$ away from the Ancortex radar. . . . . . . . . . . . . . . . . . . . 38

16 Short windowed STFT micro-Doppler signatures of multi-rotor and single rotor drones. . . . . . . . . . . . . . . . . . . . 39

17 STFT spectrogram of the fixed wing drone captured with the Xethru X4 radar showing HERM lines. . . . . . . . . . . . . . . . . . . . . . 40

18 STFT spectrogram of a waving hand and a micro-helicopter drone using the same window period of $0.064 \mathrm{~s} . \quad$. . . . . . . . . . . . 41

19 STFT of HERM lines generated using the HERM line model. . . . . . 43

20 Diagram of rotating propeller with respect to the radar line of sight. . 44

21 Simulated HERM line signature. . . . . . . . . . . . . . . 56

22 Mean and variance of the estimate of the number of harmonic components using MDL, AIC and Radoi. . . . . . . . . . . . . . . 57

23 Mean estimate using MDL with varying covariance matrix sizes for a case of two fundamental frequencies. . . . . . . . . . . . . . . . 59

24 Left: Spectrogram. Right: Estimated number of harmonic components using Radoi method on the time series as compared to the spectrogram peak detection method. . . . . . . . . . . . . . . . . 60

25 Estimated number of harmonic components using Radoi and MDL for Hexacopter with multiple fundamental frequencies. . . . . . . . . 61

26 Distribution of eigenvalues with the main Doppler component $a_{0}$ and without. . . . . . . . . . . . . . . . 63

27 Performance of proposed rotary wing detector with and without removal of the main Doppler component. . . . . . . . . . . . . . . 64

28 STFT Spectrogram. Left: HERM line spectrogram of the microhelicopter drone with its rotor spinning, starting from $0.5 \mathrm{~s}$ and stopping at 7 s. Right: Stationary micro-helicopter drone with its rotors at rest. . . . . . . . . . . . . . . . . . 
29 Estimated number of harmonic components using MDL detector with varying window size and a $30 \times 30$ covariance matrix. . . . . . . . . 67

30 Estimated number of harmonic components using MDL with varying the size of the covariance matrix. . . . . . . . . . . . . . . 68

31 Spectrogram-based detection method. Left: histogram power distribution and Right: ROC curve. . . . . . . . . . . . . . . . . . . . 69

32 Histogram distribution for MDL-based detector using $\mathrm{M}=30$ and $\mathrm{L}=128$. 69

33 2-D radar matrix showing the CFAR detections and the detections using the MDL detector. . . . . . . . . . . . . . . . 70

34 Cepstrogram and cepstrum. . . . . . . . . . . . . 75

35 Cadence-velocity diagram and cadence-velocity spectrum of micro-Doppler captured by the TI radar. . . . . . . . . . . . . . . . . 76

36 Pattern of harmonics in log frequency scale. . . . . . . . . . . . . 78

37 Processing steps of the log harmonic summation algorithm. . . . . . . 79

38 Steps for fundamental frequency estimation using MUSIC. . . . . . . 83

39 Simulated HERM line signatures. . . . . . . . . . . . . . . . 85

40 Histogram distribution of fundamental frequency estimation using the three methods on simulated data at an SNR of $7 \mathrm{~dB}$. . . . . . . . . 87

41 HERM line signature of single rotor micro-helicopter drone with rotor spinning at constant speed. . . . . . . . . . . . . . . . . 88

42 Histogram distribution for estimated fundamental frequency of single rotor micro-helicopter using non-overlapping window of 512 samples.

43 Fundamental frequency estimate using a window of 128 samples for single rotor micro-helicopter. . . . . . . . . . . . . . . . . . . 90

44 HERM line signature of flying DJI Spark quadcopter at $4 \mathrm{~m}$ generated using Hann window size of 512 samples with $80 \%$ overlap. . . . . . . 92

45 HERM line spectrum of flying DJI Spark quadcopter generated using a FFT of 128 samples (Left) and 256 samples (Right). The red points represent the frequencies estimated by root-MUSIC. . . . . . . . . . . 92

46 HERM line spectrogram comparison of three types of drones. . . . . . 94 
47 Histogram distribution of the number of HERM lines and the fundamental frequency for the three types of drones. . . . . . . . . . . . . 95

48 Chirp waveform in time and frequency domain . . . . . . . . . . . . 110

49 Block diagram of FMCW radar [48] . . . . . . . . . . . . . . . . . . 111

50 (Top) The red chirp represents the transmitted wave while the blue chirp represents the received wave. The mixed signal is shown below showing the beat frequency. The doppler shift denoted as $f_{d}$. . . . 113

51 2D FFT processing of FMCW radar [48] . . . . . . . . . . . . . . . 115

52 Xethru X4 Radar. . . . . . . . . . . . . . . . . . . 116

53 Radar frame and the downsampled baseband frame [50]. . . . . . . . 117

54 Chirp frame structure [52] . . . . . . . . . . . . . . . . . . . . 119

55 Sensor config in mmwave studio showing all the parameters that can be modified. . . . . . . . . . . . . . . . . . . . . . . . 121

56 2-piece radar setup. The data capture board is connected to the AWR1642 radar board using a ribbon cable. . . . . . . . . . . . . . . 122

57 I/Q radar data for 4 receivers. . . . . . . . . . . . . . . . . . . 122

58 Range FFT spectrogram and range Doppler spectrogram. . . . . . . . 123

59 Left: Ancortek SDR980AD2 X-Band radar specifications. Right: Ancortek SDR580 AD2 C-Band radar specifications. . . . . . . . . . . . 124

60 Radar setup for the Ancortek Radars. Both radars were operating at the same time. . . . . . . . . . . . . . . . . . . 125

61 HERM line spectrograms for DJI Spark. . . . . . . . . . . . . . . 127

62 HERM line spectrogram for DJI Spark at $8 \mathrm{~m}$ captured using the 9.8 GHz radar. . . . . . . . . . . . . . . . . . . . . . 128 


\section{List of Abbreviations}

$\begin{array}{ll}\text { DRDC } & \text { Defense Research and Development Canada } \\ \text { NRC } & \text { National Research Council Canada } \\ \text { UAV } & \text { Unmanned Aerial Vehicle } \\ \text { MAV } & \text { Micro Aerial Vehicles } \\ \text { SUAV } & \text { Small Unmanned Aerial Vehicles } \\ \text { SFOC } & \text { Special Flight Operations Certificate } \\ \text { ATR } & \text { Automatic Target Recognition } \\ \text { RCS } & \text { Radar Cross-Section } \\ \text { MTI } & \text { Moving Target Indicator } \\ \text { PRF } & \text { Pulse Repetition Frequency } \\ \text { PRI } & \text { Pulse Repetition Interval } \\ \text { STFT } & \text { Short Time Fourier Transform } \\ \text { HERM } & \text { Helicopter Rotor Modulation } \\ \text { PCA } & \text { Principal Component Analysis } \\ \text { EMD } & \text { Empirical Mode Decomposition } \\ \text { CNN } & \text { Convolutional Neural Network } \\ \text { IMF } & \text { Intrinsic Mode Function } \\ \text { LPI } & \text { Low Probability of Intercept } \\ \text { CW } & \text { Continuous Wave } \\ \text { FMCW } & \text { Frequency Modulated Continuous Wave } \\ \text { ADC } & \text { Analog-to-Digital Converter } \\ \text { UWB } & \text { Ultra-Wideband } \\ \text { SSA } & \text { Singular Spectrum Analysis } \\ & \end{array}$




$\begin{array}{ll}\text { MDL } & \text { Minimum Description Length } \\ \text { AIC } & \text { Akaike Information Criterion } \\ \text { MUSIC } & \text { MUltiple SIgnal Classification } \\ \text { ROC } & \text { Receiver Operating Characteristic } \\ \text { PDF } & \text { Probability Density Function } \\ \text { CVD } & \text { Cadence Velocity Diagram } \\ \text { CVS } & \text { Cadence Velocity Spectrum } \\ \text { IIR } & \text { Infinite Impulse Response } \\ \text { CFAR } & \text { Constant False Alarm Rate } \\ \text { SOCA } & \text { Smallest of Cell Averaging } \\ \text { MIMO } & \text { Multiple In Multiple Out }\end{array}$




\section{Chapter 1}

\section{Introduction}

\subsection{Motivation}

On May 25, 2016, a drone was spotted near the Ottawa airport by pilots of two passenger jets [1]. The airport was closed temporarily while $2 \mathrm{CF}-18$ s were scrambled to search for the UAV [1]. This search proved to be futile and the drone was never found [1]. This incident posed an important security question: "how can UAVs be detected and countered?"

In Canada, drone use is regulated under Part IX of the Canadian Aviation Regulations [2]. Flying drones under $250 \mathrm{~g}$ are unregulated but flying drones between $250 \mathrm{~g}$ and $25 \mathrm{~kg}$ require a drone licence [2]. Drones that are particularly threatening are SUAVs (Small UAVs) and MAVs (Micro Aerial Vehicles) because of their portability and easy accessibility. In Canada, MAVs are defined as drones weighing less than $250 \mathrm{~g}$ and are unregulated. SUAVs are defined as drones weighing less that $25 \mathrm{~kg}$ [3]. Drones weighing more than $25 \mathrm{~kg}$ require a Special Flight Operations Certificate (SFOC) in Canada so they pose less of a threat [4]. There are also fines for inappropriate use such 
Table 1: Comparison of drone detection methods.

\begin{tabular}{|c|c|c|}
\hline Method & Advantages & Disadvantages \\
\hline Radar & $\begin{array}{l}\text { - Does not require line of sight } \\
\text { - Longer range than visual or acoustic }\end{array}$ & $\begin{array}{l}\text { - Probability of detection limited by } \\
\text { small RCS } \\
\text { Drones can be mistaken for birds, } \\
\text { clutter }\end{array}$ \\
\hline Visual Optical & $\begin{array}{l}\text { - Low cost } \\
\text { - Can use human operators to visually } \\
\text { identify drones }\end{array}$ & $\begin{array}{l}\text { - May require many cameras/sensors } \\
\text { Ineffective when there's fog, clouds, } \\
\text { precipitation }\end{array}$ \\
\hline Acoustic & $\begin{array}{l}\text { - Low cost } \\
\text { - Works in all weather conditions }\end{array}$ & $\begin{array}{l}\text { - Requires database of drone audio } \\
\text { signatures } \\
\text { - Very short range }\end{array}$ \\
\hline RF Link & $\begin{array}{l}\text { - Long range detection } \\
\text { - Low cost } \\
\text { - Works in all weather conditions }\end{array}$ & $\begin{array}{l}\text { Crowded RF band may lead to false } \\
\text { alarms } \\
\text { Does not work for autonomous } \\
\text { drones }\end{array}$ \\
\hline
\end{tabular}

as flying close to prohibited areas such as an airport [2]. However, methods to enforce these laws and methods to defeat uncooperative drones are not well developed.

This thesis considers using radar to detect and classify these small drones as well as discern them from other aerial targets such as birds. Although several other techniques using optical, thermal or acoustic sensors are available to detect and classify drones, radar-based method is considered in this thesis. Radar-based detection and classification has several advantages over other detection techniques and notably the following advantages are unique:

1. Radar-based methods are generally immune to changes in the weather;

2. Radar-based methods can detect targets further away than other methods like acoustic or optical.

The following Table 1 compares the advantages and disadvantages of currently available technologies for drone detection and classification.

Radar detection of drones use traditional radar methods such as constant false alarm 
rate (CFAR) or moving target indicator (MTI) along with Doppler. Traditional radar-based drone detection methods may fail to detect drones that have small radar cross-section (RCS) and those that fly at low altitudes and with low speed. Often such drones can be easily mistaken for birds as drones and birds have similar characteristics [5]. For instance, drones and birds can fly with the same velocity and can have similar RCS. This makes identification of drones difficult when using Doppler as a feature.

In the case where radar RCS and Doppler features are insufficient for drone detection, micro-Doppler features can be used. Micro-Doppler signatures are unique electromagnetic echoes caused by the moving parts of the radar target. The main component of drones which induce micro-Doppler are the propellers. In the case of clutter or a gliding bird, no micro-Doppler will be present. Therefore, there is promise in this method when it comes to detecting drones. For instance, Fioranelli showed that the presence of micro-Doppler can be used to discern drones from clutter [6]. MicroDoppler can also be used to classify the types of drones. Different drones will have different micro-Doppler signatures depending on the number, speed and orientation of propellers, and propeller blade lengths.

Machine learning techniques have been applied using features from micro-Doppler signatures or using deep learning with spectrogram images to develop Automatic Target Recognition (ATR) algorithms [7]. However, naively applying deep learning techniques using spectrogram images may not be the best approach. While deep learning techniques can automatically select features, it is difficult to interpret and explain what was learnt by the classifier. Generally, micro-Doppler signatures can be related to the radar and drone specifications. It is important to study this relationship to select the most influential features for detection and classification of drones and to improve the scalability for other radar systems. Drones can also have very erratic behaviour; hence, learning from small, limited dataset may also be an issue. Generally, a large dataset would be required to achieve generalization when developing classification using deep learning. Obtaining large dataset is cumbersome. Therefore, designing a good classifier system for drones is a formidable task. 


\subsection{Problem Statement}

Micro-Doppler-based target recognition is concerned with extracting features from unique electromagnetic signatures of moving targets and applying machine learning to classify them. UAVs pose an unique radar-based detection problem due to their small RCS and low speed. They can be easily mistaken for birds or clutter. MicroDoppler features can potentially be used to filter out these false alarms as well as classify between different classes of UAVs.

Although micro-Doppler features have been proposed in the literature, most of the work in this area is based on a radar that has a high pulse repetition frequency (PRF). Unfortunately, such radars cannot be used for long range detection of UAVs. Since UAVs are threats, there is a need to detect the UAVs at longer ranges so that adequate counter-measures can be taken. This calls for use of low PRF radars. UAV detection using low PRF radars is problematic because the current techniques used by high PRF radars cannot be adapted to work at low PRF. High PRF radars use blade flashes (radar returns from the blade as they approach and move away from the radar) of UAV propeller blades in their detection/classification technique. With low PRF radars, these flashes would be distorted due to low sampling. Even if sampled adequately, frequency resolution would not be adequate to efficiently detect or classify, thus making the detection/classification of UAVs difficult. So alternate methods that exploit different kinds of micro-Doppler features may be in order for low PRF radars.

Most research on micro-Doppler analysis assume that the analysis window size is shorter than the propeller rotating period [8] [9]. This results in sinusoidal microDoppler signatures showing the rotor blade flashes. In order to achieve this, the PRF needs to be twice the micro-Doppler shift of the rotating propellers. However, flying drones have high propeller rotation rates of $100-300 \mathrm{r} / \mathrm{s}$ which can make this requirement difficult to meet [10]. While using a low PRF radar for detection, an alternative way of micro-Doppler analysis may be done using a long window which 
results in horizontal spectral lines called Helicopter Rotation Modulation (HERM) line signatures. Although HERM line signatures are well known in the literature [10] [11], such signatures have not been extensively used for UAV detection/classification. HERM lines may be a better candidate for reliable detection/classification of UAVs at long ranges. It is important to study micro-Doppler target recognition techniques using this kind of signature with low PRF radar for counter UAV measures in areas of defence and security. Therefore, this thesis will focus on the development of HERMbased detection and classification techniques of UAVs using radars.

\subsection{Contributions}

The objective of this thesis is to investigate radar detection of drones using off-theshelf low PRI radars as well as develop algorithms to classify targets using microDoppler HERM line features. The contributions of this thesis are as follows:

1. A simple model for HERM line signatures was developed based on experimental results. This allows for the simple mathematical modeling of single and multirotor type drones. The HERM line signature are decomposed into three features, namely the separation frequency (depends on the speed of the UAV), the number of HERM lines and the number of fundamental frequencies.

2. Algorithms for estimating number of HERM lines using the existing parametric spectral estimation techniques were proposed. The state-of-the-art algorithms are restricted to using peaks estimated from the spectrum obtained through Fourier analysis.

3. The HERM line separation frequency, which is related to the propeller rotation rate (and therefore speed of the UAV) was estimated using two techniques - the $\log$ harmonic summation and MUSIC. Such an estimation technique does not 
exist in the current literature. The performance of this estimation technique was compared with those of the state-of-the-art techniques in the literature such as cepstrum and cadence-velocity diagram.

4. A novel rotary drone detector using Minimum Description Length (MDL) technique was developed and analyzed experimentally.

5. A Naive- Bayes classification based on number of HERM lines was proposed for classifying three different rotary drones.

The following publications have been disseminated from thesis work

Journal Paper

- P. Klaer, A. Huang, P. Sévigny, S. Rajan, S. Pant, P. Patnaik, and B. Balaji, "An Investigation of Rotary Drone HERM Line Spectrum under Maneuvering Conditions," Sensors, vol. 20, no. 20, p. 5940, Oct. 2020.

Conference Papers

- A. Huang, P. Sévigny, B. Balaji, and S. Rajan, "Fundamental frequency estimation of HERM lines of drones," in 2020 IEEE International Radar Conference (RADAR), 2020, pp. 1013-1018.

- A. Huang, P. Sévigny, B. Balaji and S. Rajan, "Radar Micro-Doppler-based Rotary Drone Detection using Parametric Spectral Estimation Methods," 2020 IEEE Sensors, Rotterdam, 2020, pp. 1-4.

$\underline{\text { Report }}$

- A. Huang, B. Balaji, P. Klaer, "Radar Reference Guide: Xethru X4 and TI AWR1642," Defence Research and Development Canada Scientific Report, D20-210-03364, Ottawa Research Centre, January 2020.

$\underline{\text { Reports to be submitted }}$

- I. Lam, A. Huang, S. Pant, D. Klisch, "NRC Drone Trials", National Research Council of Canada Report, Aerospace Research Centre. 
- A. Huang, S. Rajan, B. Balaji, "Radar Performance Prediction Report," Defence Research and Development Canada Scientific Report, Ottawa Research Centre.

- A. Huang, P. Klaer, S. Rajan, B. Balaji, "Radar Signal Processing for micro-Doppler Analysis," Defence Research and Development Canada Scientific Report, Ottawa Research Centre.

\subsection{Limitations of the Thesis}

The following are the limitations of the work reported in this thesis

- The scope of this thesis is limited to experiments mostly in a controlled indoor environment due to COVID-19 pandemic restrictions. The drones were always secured on a stand and therefore, they were never moving or flying but were only hovering. However, the speed of the rotors were changed to simulate maneuvering behavior. As only one drone was used at a time, the effects of drone swarming was not studied in this work.

- Two types of radars, the Xethru X4 radar and the TI AWR1642 radar, each operating at different frequency, were used in this study. The Xethru X4 radar was operating at $7.29 \mathrm{GHz}$ while the TI AWR1642 radar was operating at 77 GHz. These two radars were short range radars. Only Xethru X4 was a low PRF radar and TI radar had only capability of short data collections.

- Due to the lack of data, using machine learning techniques to evaluate the performance of these features was limited. The results presented in this thesis are very preliminary and may be too restrictive. Therefore, only limited inferences can be made in the classification aspect of this thesis work. 


\subsection{Thesis Overview}

Chapter 2 presents some basic radar theory and provides an overview of the state of art in micro-Doppler classification of UAVs.

Chapter 3 shows experimental micro-Doppler signatures of different drones. Based on these results, a simple mathematical description of HERM line signatures was developed with 3 proposed features.

Chapter 4 describes the use of subspace-based spectral estimation methods to estimate the number of HERM lines and compares with a spectrogram peak detection method. The techniques used are shown in Figure 1.

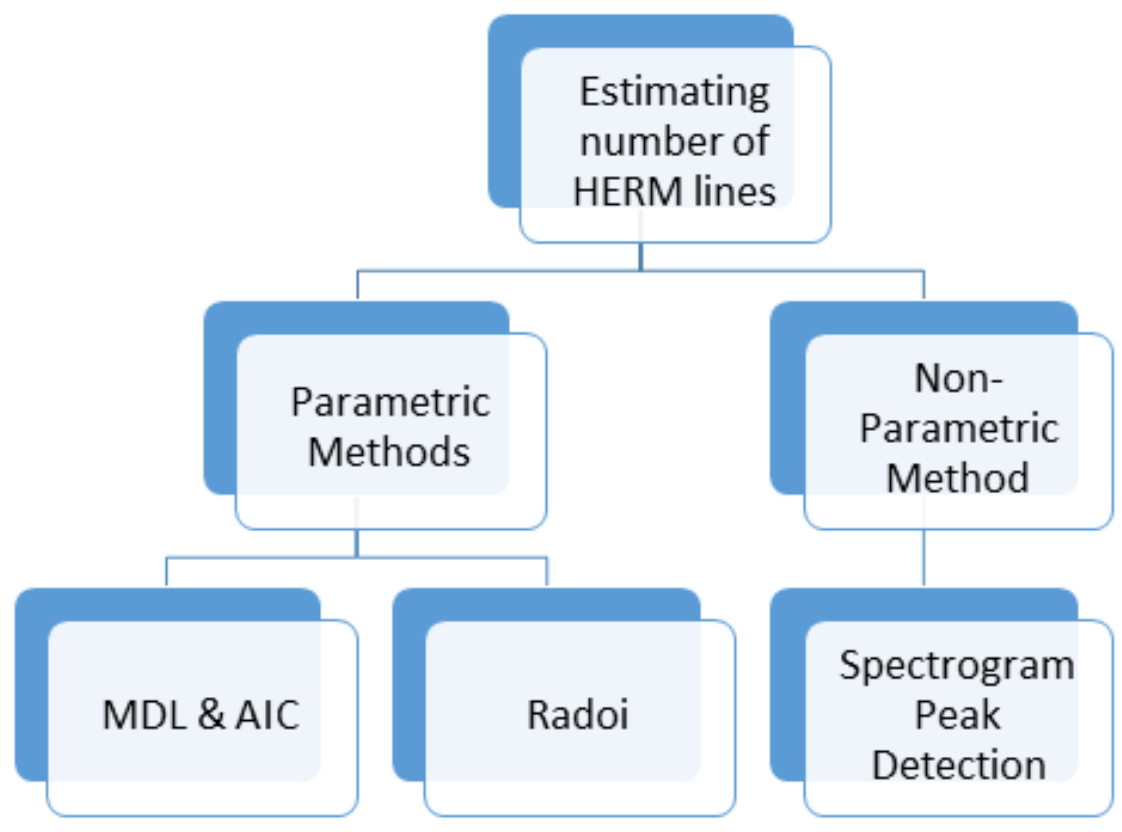

Figure 1: Techniques for estimating number of HERM lines.

Chapter 5 describes 2 methods of estimating the fundamental frequency using the HERM line spectrum. A comparison of the proposed methods with other popular 
Fourier based techniques is also presented. Figure 2 lists all the techniques utilized in that chapter.

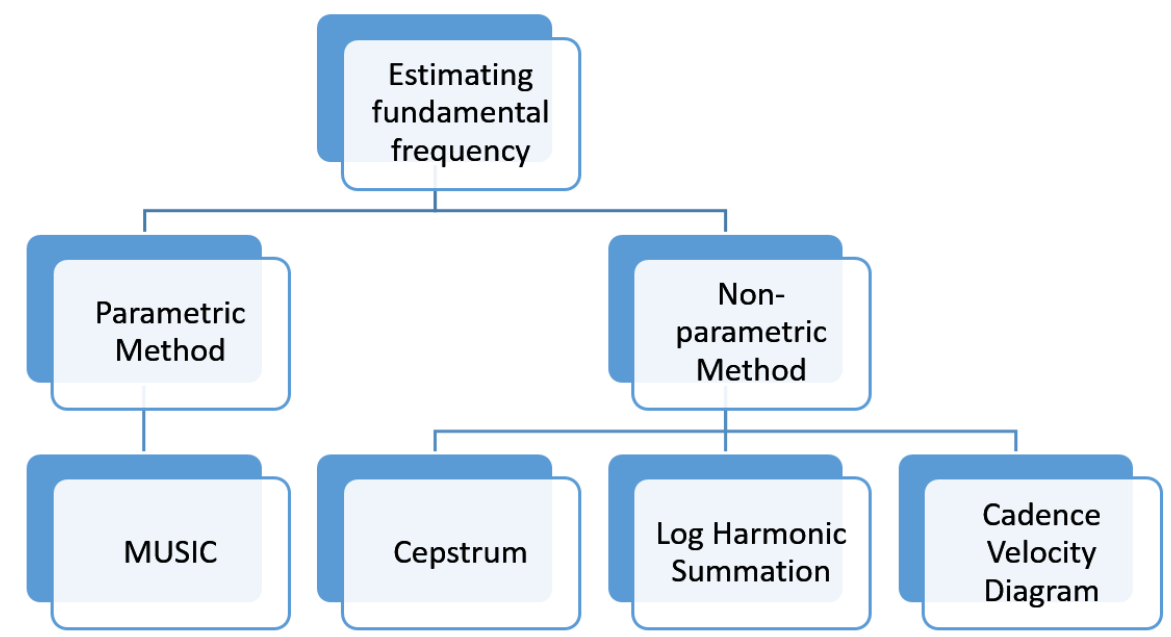

Figure 2: Techniques for estimating the micro-Doppler fundamental frequency.

Chapter 6 concludes the thesis by summarizing the contributions and providing direction for future work. 


\section{Chapter 2}

\section{Background}

This chapter provides an introduction of radars and the radar systems used in this thesis. It also covers the concept of micro-Doppler and motivates the advantages of HERM line signatures. Finally, a literature review of micro-Doppler-based drone classification is provided.

\section{$2.1 \quad$ Radar Basics}

Radar, an acronym for RAdio Detection And Ranging, was first developed by German inventor Charles Hulsmeyer in 1904 who utilized radio echoes to detect the presence of ships up to $3 \mathrm{~km}$ away. However, radar technology did not take off until WWII where it was instrumental in providing an early warning system during the Battle of Britain. Radar detects targets by sending electromagnetic pulses and analyzing the received echoes as the pulses reflect off objects in the environments. EM waves travel at the speed of light so the time required to travel from the target and back is defined 
as:

$$
\tau=\frac{2 R}{c}
$$

where $R$ is the distance from the target to the radar and $\mathrm{c}$ is the speed of light. Figure 3 shows a monostatic radar setup consisting of a co-located transmitter and receiver antenna.

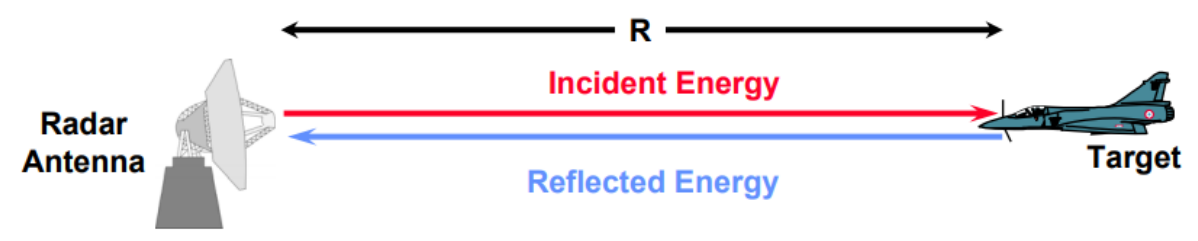

Figure 3: Monostatic radar setup [12].

The power density, $P_{d}$, of the directional transmit antenna is:

$$
P_{d}=\frac{P_{t} G_{t}}{4 \pi R^{2}},
$$

where $P_{t}$ is the transmit power, $G_{t}$ is the gain and $\mathrm{R}$ is the distance away from the antenna. When the radar transmitted wave hits a target, some portion of the energy is reflected back. The amount of power reflected back depends on the radar crosssection (RCS) area $\sigma$ of the target measured in $\left(\mathrm{m}^{2}\right)$. Drones have very small RCS of less than $0.01 \mathrm{~m}^{2}$ which makes radar-based detection very difficult. The power of the reflected pulse at the receiver is:

$$
P_{r}=\frac{P_{t} G_{t}}{4 \pi R^{2}} \frac{\sigma A_{e}}{4 \pi R^{2}},
$$

where $A_{e}$ is the antenna aperture of the receiver antenna denoted by:

$$
A_{e}=\frac{\lambda^{2} G_{r}}{4 \pi},
$$

where $\lambda$ is the wavelength of the radar carrier frequency and $G_{r}$ is the receiver gain.

Thus, the total reflected power at the receiver becomes:

$$
P_{r}=\frac{P_{t} G_{t}}{4 \pi R^{2}} \frac{G r \lambda^{2}}{4 \pi} \frac{\sigma}{4 \pi R^{2}} .
$$


For the monostatic radars used in this thesis, the transmitter gain is equal to the receiver gain $\left(G_{t}=G_{r}\right)$. Since the transmitted wave needs to travel to the target and back, the power density at the receiver falls by $R^{4}$. The following equation can be used to estimate the maximum detectable range for given a minimum detectable received power.

Moreover, real radar systems are also be affected by system noise and thermal noise. System noise is caused by losses in the electronic components of the radar system and is denoted by L. Thermal noise is inherent in all electronic systems and uniform across all frequencies. For a radar system with limited bandwidth, the thermal noise is given by:

$$
P_{\text {ThermalNoise }}=k T B
$$

where $k$ is the Boltzmann constant, $T$ is the system temperature in Kelvins and $B$ is the bandwidth. In order to detect a target, the power of the received pulse must exceed this noise. Equation 5 can be rewritten as the ratio of the input power to noise, or the signal to noise ratio (SNR).

$$
\begin{aligned}
S N R & =\frac{P_{r}}{k T B L} \\
& =\frac{P_{t} G_{t}}{4 \pi R^{2}} \frac{G r \lambda^{2}}{4 \pi} \frac{\sigma}{4 \pi R^{2}} \frac{1}{k T B L}
\end{aligned}
$$

The SNR allows for the universal comparison of the detection performance of different radars.

\subsection{Doppler Frequency}

Radar is also able to measure velocity using the Doppler shift. The Doppler shift was first described in 1842 by physicist Christian Doppler. The frequency of an observed wave differs from the frequency of the emitted wave due to the change in the target's velocity. This apparent shift in the frequency is referred to as the Doppler shift. 
Objects moving towards the radar will appear to have an increasing frequency while objects moving away will appear to have a decreasing frequency. The relationship between the observed frequency, $f_{r}$ and the emitted frequency, $f_{t}$ is:

$$
f_{r}=f_{t}\left(\frac{c \pm v_{r}}{c \pm v_{s}}\right)
$$

where $c$ is the speed of light, $v_{r}$ is the radial velocity of the moving object and $v_{s}$ is the radial velocity of the observer. Since the radar emitter is not moving $\left(v_{s}=0\right)$, then the equation can be simplified into:

$$
f_{r}=f_{t}\left(1+\frac{v_{r}}{c}\right) .
$$

The change in frequency, $\Delta f$, due to the Doppler shift is:

$$
\Delta f=\frac{v_{r}}{\lambda} .
$$

For a monostatic radar, the total Doppler shift is actually twice the one-way Doppler shift:

$$
f_{d}=\frac{2 v_{r}}{\lambda}
$$

since there will be an additional Doppler shift from the reflected pulse at the receiver.

Radar returns for every range bin ("fast time") is obtained for each pulse in a pulse repetition interval (PRI). The Doppler shift is estimated in radar processing by taking the Fourier transform of the returned radar signal over multiple pulses in a specific range ("slow time"). Figure 4 shows a two dimensional radar data matrix with range bins in fast time (along x-direction) and slow time bins (y-direction).

For pulse-Doppler radars, the PRI of a radar affects both the maximum unambiguous range and Doppler measurements. Range is measured by the time delay between the received and transmitted pulse. Thus, if the received pulse returns of previous pulse are received after the transmission of the following pulses, then the range is 


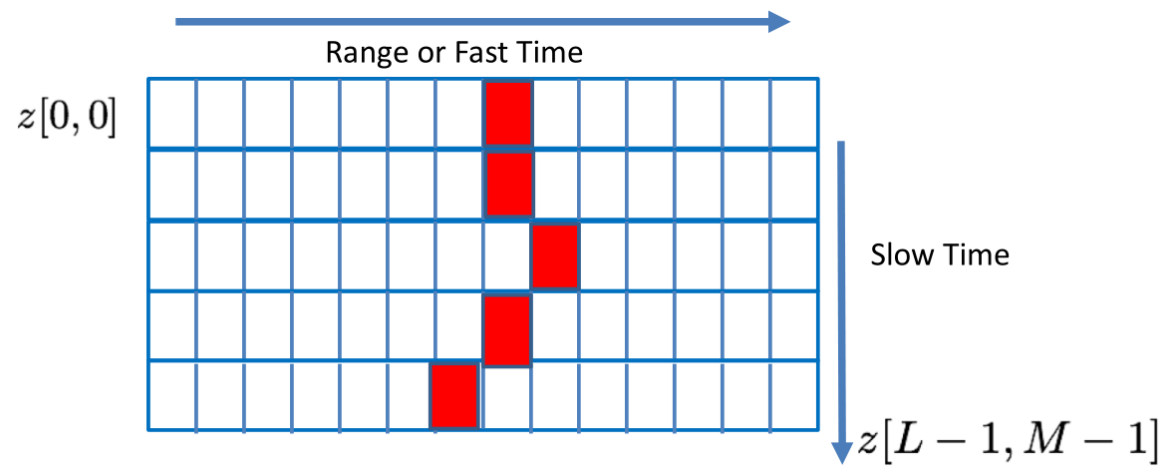

Figure 4: Single channel radar data matrix.

ambiguous. The maximum unambiguous range of a pulse-Doppler radar is given by:

$$
\begin{aligned}
R_{\text {max }} & =c \frac{\mathrm{PRI}}{2}, \\
& =\frac{c}{2 \mathrm{PRF}}
\end{aligned}
$$

The maximum unambiguous velocity is given by noting that the unambiguous Doppler cannot exceed the PRF:

$$
\begin{aligned}
v_{\max } & =\frac{\lambda \mathrm{PRF}}{2} \\
& =\frac{\lambda}{2 \mathrm{PRI}}
\end{aligned}
$$

where PRF (Pulse Repetition Frequency) is $\frac{1}{\text { PRI }}$. From these equations, one can see that there is a trade-off between the unambiguous range and the Doppler. A longer range radar would have a longer PRI meaning the maximum unambiguous velocity would be lower. This trade-off is especially problematic for drone micro-Doppler analysis since the Doppler induced by their spinning propellers can be very high. 


\subsection{Pulse Compression}

Another factor which influences radar drone detection is the radar range resolution. For pulsed radars with rectangular pulses, the range resolution is directly proportional to the the pulse width $(T)$ and inversely proportional to the bandwidth $\left(\frac{1}{T}\right)$.

$$
\begin{aligned}
\Delta r & =\frac{c T}{2} \\
\Delta r & =\frac{c}{2 B}
\end{aligned}
$$

Equation (15) is true for all radars. Modulation enables one to have large duration pulses with high resolution, reducing the transmit power requirements. Fine range resolution is especially important for drone detection as drones are small. However, from the radar range equation, for a given SNR, higher transmit power is required if the pulse has a larger bandwidth.

Limiting the transmit power of a radar is important for low probability of intercept (LPI) purposes. When radars transmit high power, an adversary can intercept the transmitted radar signal and can potentially jam the radar. The process of modulating a longer duration pulse to achieve resolution performance equivalent to an unmodulated rectangular pulse is termed as pulse compression. Pulse compression allows for the same range resolution as that of a short pulsed radar but at the expense of using a much longer pulse duration. As noted above, this is achieved by modulating the longer duration pulse in frequency or phase. In the next section, we introduce frequency modulated continuous wave (FMCW) radars which take advantage of pulse compression. The FMCW radars enable LPI performance relative to pulsed radar, while maintaining the advantages of pulse compression. 


\subsection{Frequency Modulated Continuous Wave Radars}

Frequency modulated continuous wave (FMCW) radars transmit frequency modulated sinusoidal waveforms that sweep in ascending frequency called a chirp. As shown in [13], [14], range in FMCW radars is determined by the frequency difference between the transmitted and received chirp, since this frequency difference is proportional to the time delay. The range resolution is now dependent on the chirp bandwidth rather than the chirp duration. This allows FMCW radars to have fine range resolution while achieving LPI by maintaining a long chirp duration with very high duty factor (i.e., pulse duration is close to the PRI). Doppler information is found as in pulsed radars by comparing the phase of consecutively received chirps. The following fundamental equations relate the chirp parameters to radar performance. Refer to the Appendix for a detailed derivation of these equations.

$$
\begin{aligned}
R_{\text {max }} & =\frac{f_{b} T_{c} c}{2 B} \\
R_{\text {res }} & =\frac{c}{2 B} \\
V_{\text {max }} & =\frac{\lambda}{4 T_{c}}
\end{aligned}
$$

where $T_{c}$ is the chirp duration, $B$ is the chirp bandwidth, $c$ is the speed of light and

$f_{b}$ is the maximum beat frequency. The maximum beat frequency needs to satisfy the Nyquist criterion and should not be greater than half the Analog-to-Digital Converter (ADC) sampling rate.

In this thesis, data acquired from both pulsed and FMCW radar systems were used for analysis. Refer to Appendix A for more details on the specifications of the radars used and their operation. 


\subsection{Rotor Micro-Doppler Effect}

Micro-Doppler is defined as small modulations in Doppler caused by micro-motion superimposed on the bulk motion of the target such as mechanical vibrations or rotations [15]. It was first introduced in LADAR (Laser Detection and Ranging) systems where small vibrations could induce high Doppler shifts [15]. The micro-Doppler shift is simply the Doppler shift induced by the rotational or vibration motion.

$$
f_{D}=\frac{2 v_{r}}{\lambda} .
$$

From this, it is evident that a higher Doppler shift would be induced with a shorter wavelength (or equivalently, a higher radar operating frequency).

The main part of a drone that induces micro-Doppler are its rotating blades also referred to as rotors. Radar backscatter from rotating blades were first modelled in the context of rotating blades of aircraft propellers [16]. Specifically, each blade was modelled as a rigid, homogeneous linear antenna. Each point on the antenna away from the center hub induces a Doppler shift, the largest being at the blade tip given as:

$$
f_{D}=\frac{4 \pi L \Omega \cos (\phi)}{\lambda}
$$

where $L$ is the length of the blade, $\Omega$ is the rotation rate, $\lambda$ is the wavelength and $\phi$ is the look angle of the radar with respect to the propeller. If a propeller with flat blades is spinning perpendicular to the radar LOS (Line of Sight), $\left(\phi=\frac{\pi}{2}\right)$ no micro-Doppler shift will be induced. As the rotor is spinning towards and away from the radar, this micro-Doppler shift will also change in this periodic fashion. Thus, the rotational motion of a spinning rotor imparts a periodic modulation on the bulk Doppler shift of the drone. 


\subsection{Micro-Doppler Signatures}

A micro-Doppler signature is the time-frequency representation of the change in micro-Doppler over time. Micro-Doppler signatures are typically generated by applying time-frequency transforms, like the short time Fourier transform (STFT), the Gabor transform or the Wigner-Ville distribution over samples in slow time in the target's range bin (implicitly it is assumed that the target remains in the same range bin). The Gabor transform is simply a STFT using a Gaussian window function. The Gabor transform and STFT are cross-correlation-based transform while the WignerVille distribution is auto-correlation-based transform. The Wigner-Ville Distribution has better resolution than the STFT but suffers from cross-term interference due to the auto-correlation operation.

In the case of a spinning propeller, the micro-Doppler signature will capture the periodic change in Doppler as the blade spins towards and away from the radar. A micro-Doppler analysis of the return signal could help in the characterization of drones.

\subsubsection{STFT Spectrogram}

The most popular method of visualizing micro-Doppler is using the short-time Fourier transform (STFT). A STFT spectrogram of a time series $x(n)$ is as given below.

$$
X(m, \omega)=\left|\sum_{n=-\infty}^{\infty} x(n) w(n-m) e^{-j \omega n}\right|^{2}
$$

where $w(n)$ is the window function centered at time index $m$.

The length of the window function will determine the time-resolution of the spectrogram. 


\section{Short-Window Spectrogram}

If the window size is at least half the rotation period of the propeller then a wideband spectrogram is produced showing the periodicity of the rotating blades in time. This is called the blade flash phenomena. Unique signatures would be generated depending on the number of propellers, number of blades, the rotation rate, and the blade length. An example is shown in Figure 5.

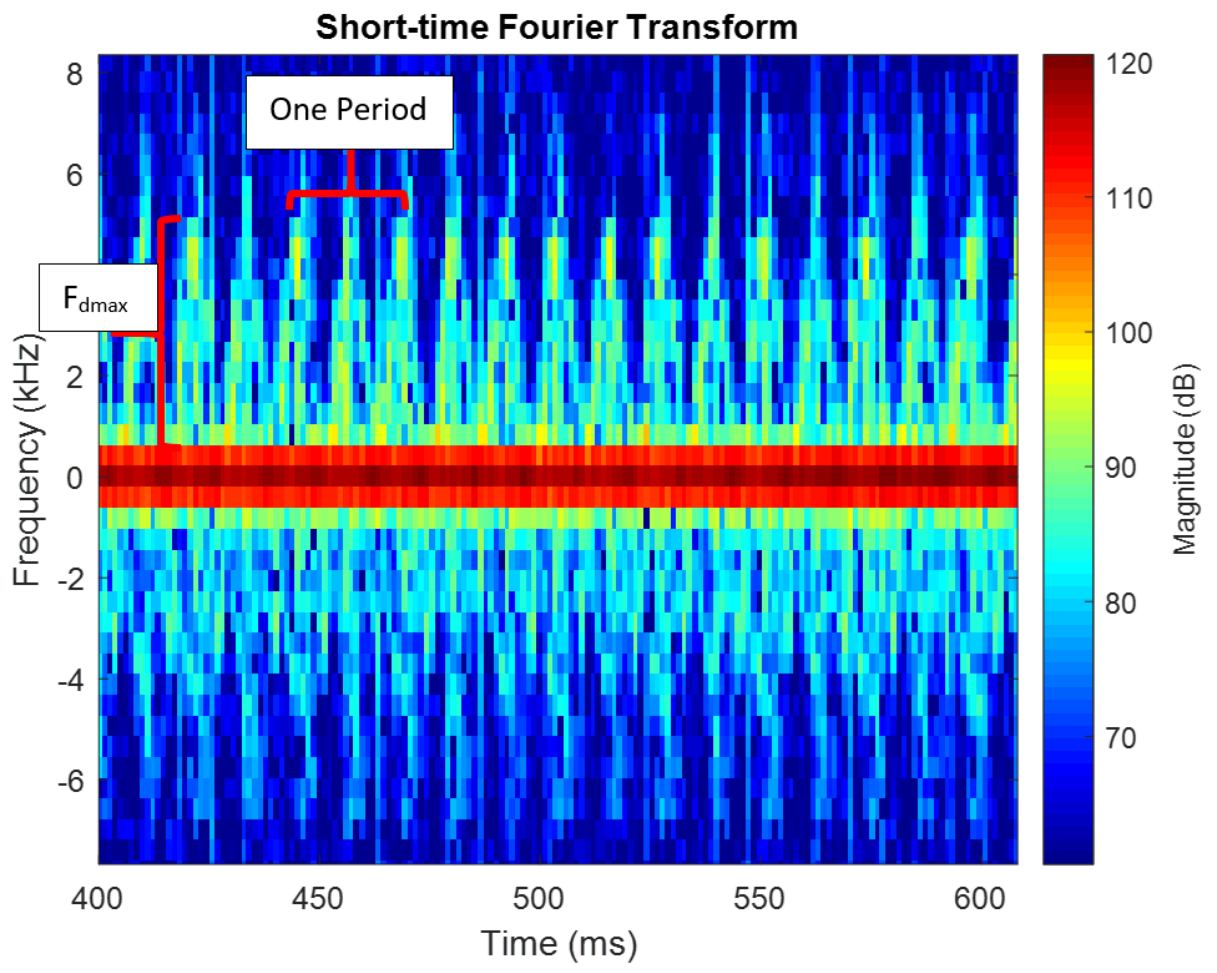

Figure 5: Micro-Doppler spectrogram of a two bladed helicopter with a 6 inch diameter propeller and a rotation rate of 34 revolutions per second measured using the TI FMCW radar.

Many features such as the rotation rate, the number of blades, the blade length can be extracted from the micro-Doppler spectrogram. A positive Doppler shift is induced 
when the propeller blade is approaching the radar and a negative Doppler shift is induced when the propeller blade is receding.

However, in order to capture this blade flash phenomenon, two requirements must be satisfied.

- The first requirement is that to prevent aliasing, the minimum PRF of the radar must be twice the maximum Doppler shift induced by the spinning propellers.

- The second requirement is that the STFT window size must be less than the rotation period of the propeller.

The first requirement can be mathematically written as follows:

$$
P R F_{\text {min }} \geq \frac{2 \times 2 \times(2 \pi l \Omega \cos (\phi))}{\lambda}
$$

where $\Omega$ is the rotation rate, $\lambda$ is the wavelength, $l$ is the blade length and $\phi$ is the angle between the radar line of sight and the rotating plane of the propeller.

Flying drones have very fast propeller rotation rates of 100 to 300 revolutions per second $(\mathrm{r} / \mathrm{s})$ which can make the PRF requirement difficult to meet. This minimum PRF requirement is also dependent on the wavelength of transmission. A radar at a higher carrier frequency will be more sensitive to Doppler shifts, so the PRF required will be higher. Figure 6 shows a graph of the required PRF as a function of frequency for a DJI Mavic Pro with a $10 \mathrm{~cm}$ long propeller and a rotation rate of $80 \mathrm{~Hz}$. DJI Mavic Pro is a popular drone used by UAV enthusiasts.

For the second requirement, if the number of samples in a window is too small, the frequency resolution will be poor and the blade flashes will be smeared. An example is shown in Figure 7 using data collected with the X-band radar with sampling rate of $1.5 \mathrm{KHz}$ (details given in the Appendix) and a STFT window size of 16 samples. 


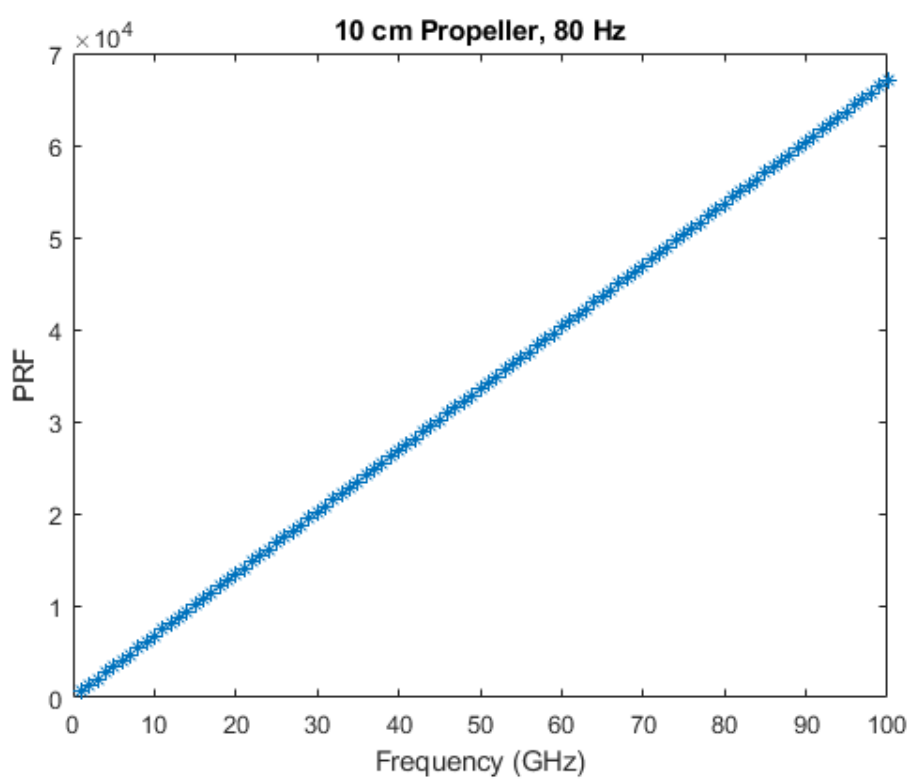

Figure 6: The minimum PRF required for unambiguous sampling of the Doppler shift of a $10 \mathrm{~cm}$ propeller spinning at $80 \mathrm{~Hz}$ as a function of carrier frequency.

This is less than the period of a spinning propeller at $50 \mathrm{r} / \mathrm{s}$. As the FFT size (16 samples) is too small, the blade flash phenomenon cannot be seen clearly.

\section{Long-Window Spectrograms}

An alternative way of visualizing micro-Doppler signatures when the PRF of the radar is insufficient is by using a long windowed STFT that spans multiple rotation periods. A narrowband spectrogram is formed where the periodicity of the spinning propellers is represented by horizontal spectral lines. The separation frequency between these spectral lines is $N$ times the propeller rotation rate where $N$ is the number of blades [11]. In the literature, these are called HERM (HElicopter Rotation Modulation) lines. HERM lines can be resolved as long as the window size covers at least two 


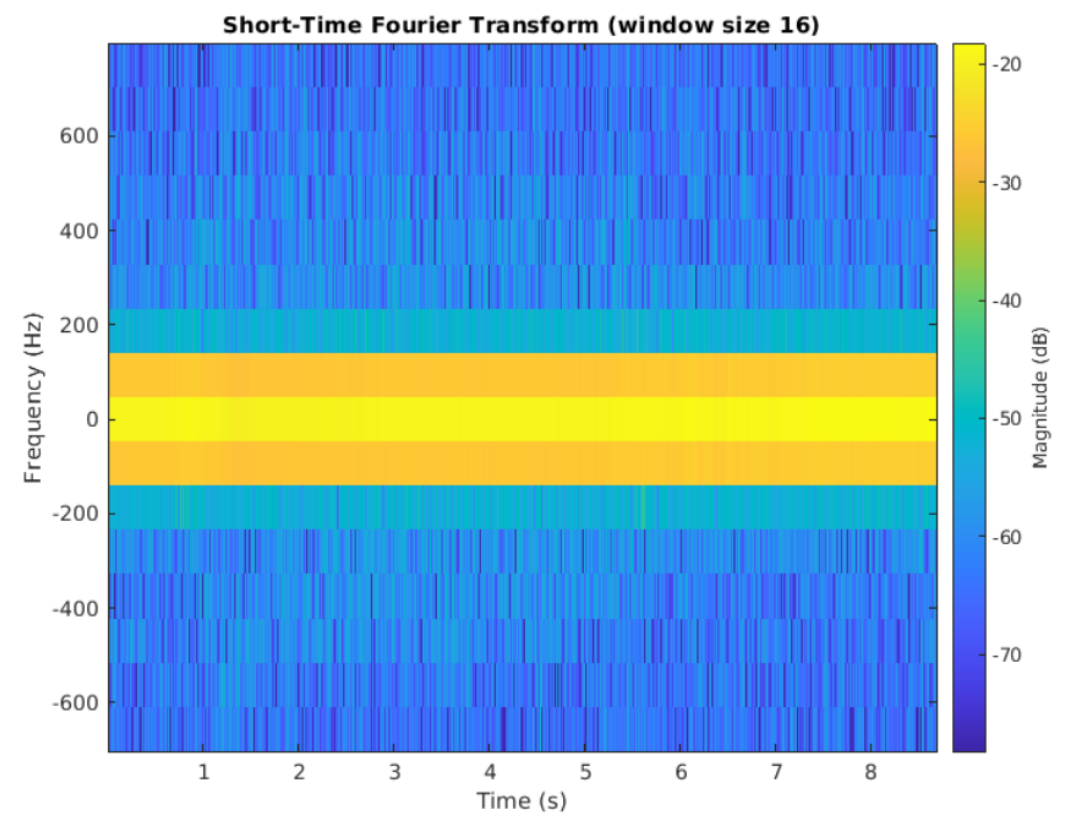

Figure 7: Spectrogram smearing due to small STFT window size of 16.

rotation periods, that is,

$$
P R F>2 \times N \Omega
$$

where $\Omega$ is the rotation rate in rotations per second $(\mathrm{r} / \mathrm{s})$. A more precise examination of this issue is available in [17].

The HERM line separation does not depend on the Doppler frequency, but rather on the propeller rotation rate. The propeller rotation frequency is much smaller than the blade tip Doppler frequency. Therefore, the PRF requirements for less stringent. For example, the DJI Mavic Pro has a hovering rotation rate of $4900 \mathrm{rpm}$ which means each rotation period is only $12 \mathrm{~ms}$. As long as the window size is at least $24 \mathrm{~ms}$, then it would be possible to resolve HERM lines. Using a longer window ( $>24 \mathrm{~ms}$ ) generally improves the frequency resolution and results in more accurate feature extraction as shown in the figure below.

Since the spectrum is a function of the periodicity and not the tip velocity, the PRF 

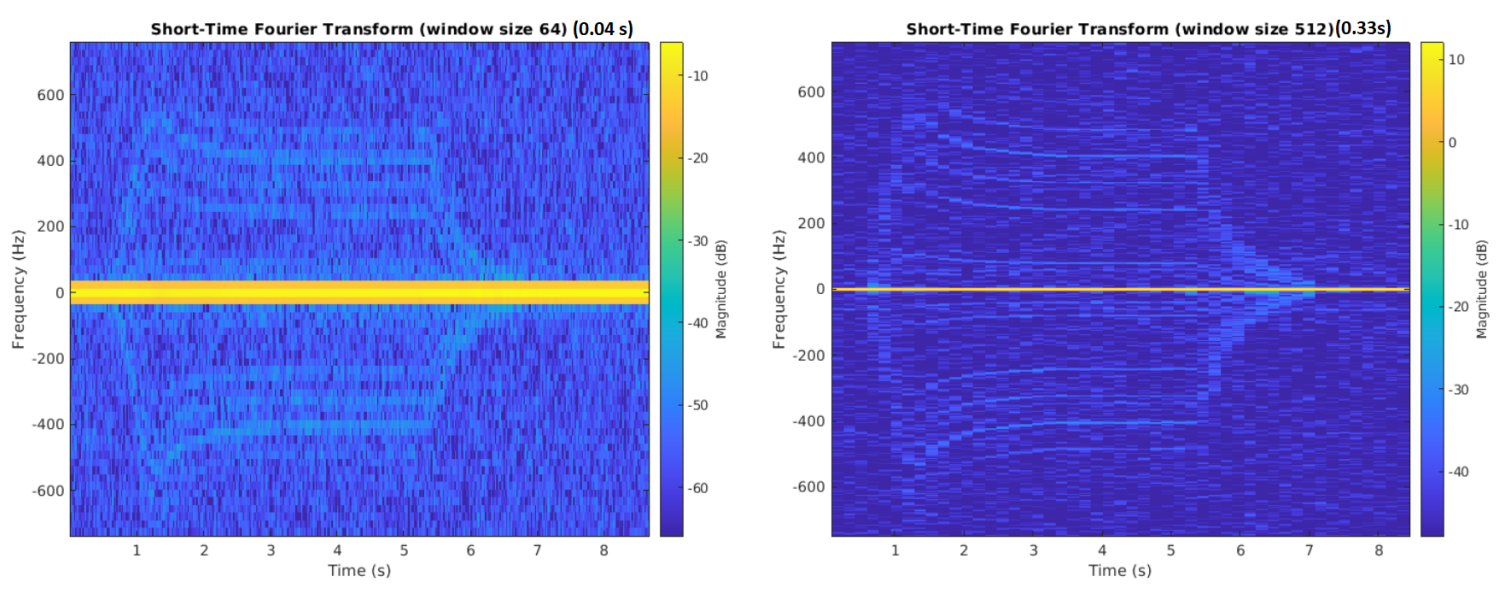

Figure 8: HERM line spectrogram generated from data collected using X-band radar with a sampling frequency of $1.5 \mathrm{KHz}$. Left: Window size of $64(\approx 0.04 \mathrm{~s})$, Right: Window size of $512(\approx 0.33 \mathrm{~s})$.

requirement in Equation 22 can be more relaxed. The PRF of the radar only needs to be twice the rotation rate. For flying drones with a propeller rotation rate of 100-300 $\mathrm{r} / \mathrm{s}$, the minimum PRF for HERM line analysis is only $200-600 \mathrm{~Hz}$. This is the motivation for using HERM lines as features since long-range low PRF radars are widely employed.

Early identification of drones with low PRF radars is definitely an advantage when it comes to defence and public safety. The feasibility of obtaining HERM line signatures at a long range was demonstrated by Cai using a low PRF radar of $1 \mathrm{KHz}$ and a 256 sample window. In his work, HERM line signatures were obtained from a DJI M600 hexacopter flying $9 \mathrm{~km}$ away from a stationary S-band radar [18]. The following section will present the state-of-the-art in drone identification using micro-Doppler. 


\subsection{Literature on Drone Classification using Micro- Doppler}

This section presents the state of the art in research in the field of drone classification using micro-Doppler features. In most cases, researchers generate their own datasets from experimental results using different drones and radars. Most experiments are conducted in close ranges of less than $60 \mathrm{~m}$, or even just at fixed distances in a controlled lab environment [7]. Depending on the PRF of the radar, the researcher then chose to either work with the short-window or long-window spectrogram methods. Notable exceptions include the work shown in [18]. His work was conducted outside of the laboratory setting for HERM line signature detection at long ranges $(9 \mathrm{~km})$. In the following sub-sections, a literature survey of feature extraction techniques that have been used for micro-Doppler-based drone classification is presented.

\subsubsection{Short-window STFT Spectrogram Methods}

Figure 5 suggests that features such as the propeller rotation period and blade length can be extracted from the periodic blade flashes in the short-window STFT spectrogram. However, extracting these features require accurate background subtraction of noise and may not be effective for weak micro-Doppler signatures. After background subtraction, Molchanov [19] used the maximum micro-Doppler shift and the rotation period to estimate the blade length as given by Equation 24 and used the blade length as a feature to classify five different drones using a support vector machine (SVM).

$$
L=\frac{V_{t i p}}{2 \pi \Omega}
$$

However, the probability of correct classification was only $59 \%$ since the drones used in the work had similar blade lengths. This also suggests that using just the blade length as a feature is not sufficient for classification of drones. 
To combat the difficulty of selecting and estimating features from the short-window STFT spectrogram, an alternative method is employed that treats the spectrogram as a matrix. Every element in the matrix is treated as a feature. Many authors have applied PCA (Principal Component Analysis) to spectrograms to reduce the dimensionality and to reveal the most influential features [19], [20]. PCA is an unsupervised method of feature space reduction that uses the eigenvalues and eigenvectors of the covariance matrix of the dataset. The eigenvalues indicate the variance in the dataset captured by each of the principal components. A projection matrix made of the eigenvectors corresponding to the highest eigenvalues is used to generate the new feature set [20]. Zheng et al. applied PCA to spectrograms of micro-Doppler signatures captured from two radar sensors (X-band and K-band) and used SVM to classify three different drones [20]. It was shown that combination of features from the two sensors yielded better results.

\subsubsection{Modified Short-Window Spectrogram Representations}

To aid in the study of the short-window pattern, two modified spectrogram representations are commonly used. The cadence velocity diagram is obtained by taking a FFT across the time axis in every Doppler bin of the STFT spectrogram [21]. This effectively measures the repetition of the blade flashes which is referred to as the cadence.

The advantage of the cadence velocity diagram is that it is a time invariant representation, unlike the spectrogram representation, if the propeller rotation rate is constant (time invariant) [21]. Zhang [22] defined the cadence velocity spectrum which is obtained by summing up the Doppler bins at every cadence to generate a 1-D frequency spectrum. Since the drones have different rotor rotation rates, their cadence veloc-

ity spectrum have peaks at different cadences. The cadence velocity spectrum was then used in a k-means classifier to classify three different hovering drones as well as 
detecting multiple drones simultaneously.

The cepstrogram is a technique popularly used in speech processing and seismology for fundamental frequency estimation [10]. One property of signals such as the speech is that the utterances of vowels have a periodic structure, namely, pitch frequency and its harmonics. Similarly, the radar returns from the rotating propellers of the drones also have similar periodic structure which are known as HERM lines [18].

The power cepstrum is defined as the inverse Fourier transform of the log of the power spectrum.

$$
C(q f t)=\left|\mathcal{F}^{-1} \log \left(|\mathcal{F}(s(t))|^{2}\right)\right|^{2},
$$

where $\mathcal{F}$ stands for the Fourier operator and $\mathcal{F}^{-} 1$ for its inverse. Taking the $\log$ operation on the squared magnitude of the Fourier transform of speech type signals compresses the amplitudes of the harmonic peaks and the resulting spectrum can be treated as an amplitude modulated waveform which is periodic in the frequency domain. The amplitude modulation reduces the dynamic range of the spectral peaks so that the weaker components are not neglected. Taking the inverse Fourier transform will result in a peak at the period of this waveform (quefrency). This is called quefrency since it is a measure of frequency but in the time domain. The fundamental frequency can then be derived by $1 / q \mathrm{ft}$. In the case of drone micro-Doppler, this is just the propeller rotation rate.

Noll developed the concept of a short time cepstrogram by applying the short time Fourier transform and then computing the cepstrum for each segment [23].

$$
C(m, k)=\left|I F F T^{-1} \log \left(|S T F T(s(n))(m, k)|^{2}\right)\right|^{2}
$$

Fuhrmann et al [24] demonstrated that the cepstrogram was able to successfully estimate the propeller rotation rate of small UAVs. However, a detailed analysis about the effectiveness of this technique was not presented. 
Many authors have used a combination of these three spectrogram representations for drone classification, namely short-window spectrogram [24], cepstrogram [25], and CVD image[8]. Patel et al [25] classified flying drones with and without payload by transfer learning with AlexNet using short-window spectrogram, cepstrogram and CVD images individually. Transfer learning is very popular in this area of research due to the lack of data. Authors found that the CVD representation required a longer observation window than the spectrogram/cepstrogram to be effective. Kim et al [8] used merged short-window spectrogram and cadence velocity spectrogram images to classify three different drones using transfer learning with GoogLeNet. Using the merged spectrogram images rather than just the individual spectrogram images improved the classification accuracy from $89 \%$ to $94 \%$. However, their work only dealt with drones at idle captured using a Ku-band FMCW radar in an anechoic chamber.

\subsubsection{Long-Window HERM line-based Methods}

Classification using HERM lines signatures have not been as extensively explored as the periodic blade flash representation of micro-Doppler in the short-window analysis. Fioranelli et al. [26] classified drones with and without payload using features extracted from HERM line signatures, namely the center frequency and the center frequency spread with a Naive Bayes classifier. Cai [27] classified multi-rotor drones using features from simulated HERM line signatures, namely the frequencies and amplitudes of the HERM line components. The features used in these works were obtained using peak detection methods under relatively high SNR conditions. However, extracting these features using peak detection methods may be difficult under low SNR. The frequency of the HERM line components may also change in a flying scenario and therefore, may not be the best or stable feature that can be relied upon for classification. Rahman [11] showed that for multi-rotor drones, the HERM line signature becomes a superposition of the backscatter from all the spinning propellers. 
This can make it difficult to extract the separation frequency between the HERM lines as the HERM lines may not be constantly spaced anymore. This further complicates HERM line analysis for classification purposes. This is one of the reasons why HERM line analysis is not very well explored. Recently, Gérard et al. [28] compared the efficiency of using different micro-Doppler representations, such as the the HERM line spectrum, the cepstrum, CVD, the spectrogram to classify five different drones using a CNN. The same integration time $(0.3 \mathrm{~s})$ was used for all these representations and it was found that using the HERM line spectrum performed the best and was the most resistant against noise. However, they found that using a short integration time of $0.036 \mathrm{~s}$ vs. an integration time of $0.3 \mathrm{~s}$ reduced the classification accuracy from 0.98 to 0.93 . This is probably due to the loss of frequency resolution due to the smaller FFT length.

\subsubsection{Non-Fourier Based Methods}

Limited research have been done in non-Fourier based methods for drone short-time micro-Doppler feature extraction. Kim et al introduced the short-time MUltiple Signal Classification (MUSIC) spectrogram and compared it with the STFT and the Wigner-Ville distribution method of generating short windowed Micro-Doppler spectrograms. The short-time MUSIC method produced the clearest spectrograms but no further work has been done on this topic.

EMD (Empirical Mode Decomposition) is a recent approach for drone micro-Doppler feature extraction that does not use the STFT. EMD decomposes the radar time series into fundamental periodic components called Intrinsic Mode Functions (IMF) [29]. IMFs are found by sifting the time series to a maxima point and then interpolating with a cubic spline. The IMF is then subtracted from the original signal and the process is repeated to find the subsequent IMFs. Figure 9 shows the first 8 IMFs for a single rotor blade spinning at $300 \mathrm{rpm}$ [29]. The first IMFs contain the highest 


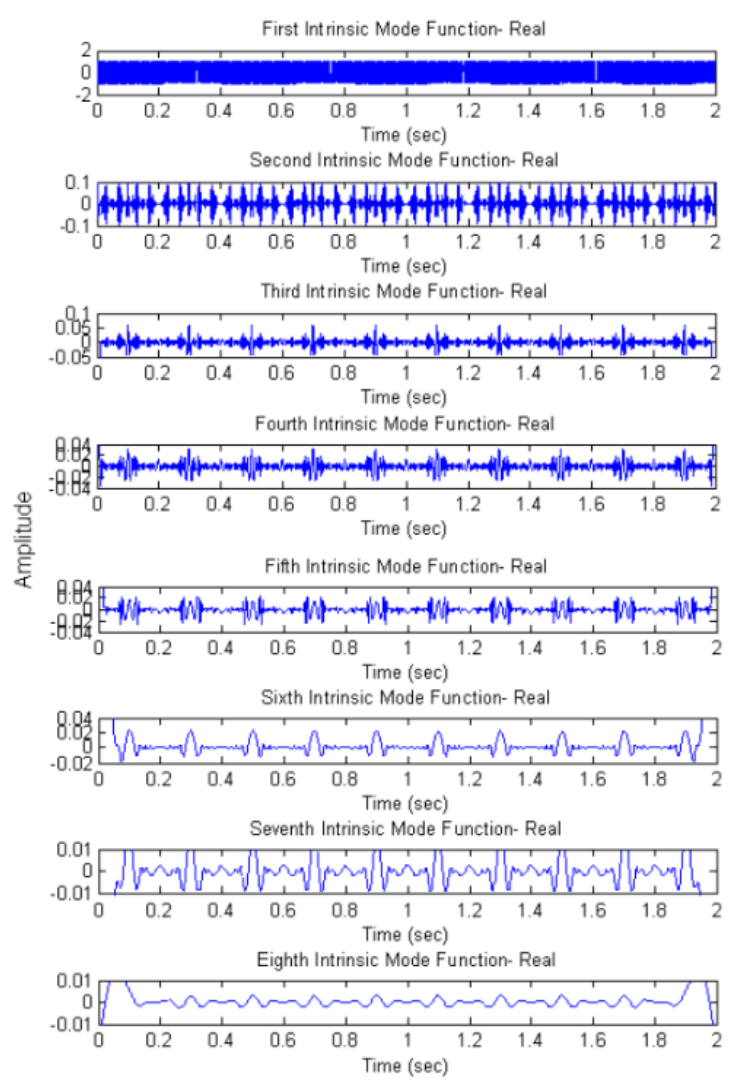

Figure 9: First 8 IMFs of a single rotor blade[29].

frequency components while the last IMFs contain the lowest oscillations. The IMFs are all orthogonal to each other meaning that filtering could be achieving by combining specific IMFs or denoising can be achieving by combining just the lower IMFs. In [30], features from the IMFs such as the zero crossing, IMF energy, standard deviation were used to distinguish different drones and birds using a SVM classifier. The classification error rate with SVM classifier using these features was compared SVM classifiers with other popular feature extraction techniques such as STFT spectrogram, cepstrum, and CVD. The EMD-derived features provided the best results. 


\subsection{Limitations and Conclusion}

Research in micro-Doppler analysis for drone identification/classification can be divided into two main research areas based on the temporal window length. The shortwindow micro-Doppler analysis is suitable when the PRF of radar is high enough to unambiguously capture the blade flashes, which limits its practical utility since most practical radars do not have the requisite PRF (else the resulting unambiguous range of the radar is too small). The long-window micro-Doppler analysis requires a lower PRF since the propeller rotation rate is typically lower than PRFs of many practical radars. There has not been as much research done in the long-window micro-Doppler analysis. This thesis aims to bridge some of the gap.

Drone classification using micro-Doppler is typically done by training classifiers using different micro-Doppler representation like the cepstrum, cadence velocity diagram and the short windowed STFT spectrogram. Recently, it was shown that using the HERM line spectrum to classify drones was superior to other micro-Doppler representations such as the cepstrum, CVD, and the short-window spectrogram given the same integration time[28]. However, using a smaller window of data of $0.03 \mathrm{~s}$ rather than $0.3 \mathrm{~s}$, the classification accuracy dropped from $98 \%$ to $93 \%$. Thus, there is a need of researching alternate methods of HERM line feature extraction to improve performance for smaller integration times. 


\section{Chapter 3}

\section{Experimental Trials and Micro-Doppler Model}

This chapter shows the micro-Doppler signatures obtained using a couple of commercial radars (two in the C-band and the other in the $\mathrm{W}$-band). These experimental results motivate the development of a simple mathematical model to characterize HERM line signatures of single-rotor and multi-rotor drones by three features, namely, the fundamental frequencies, number of HERM lines per fundamental frequency, $M$, and the number of fundamental frequencies.

\subsection{Data collection protocol}

Four different drones - the Air Hogs Havoc micro-helicopter, the DJI Spark quadcopter, the Typhoon H hexacopter and the Parrot Disco fixed wing drone - are examined in this thesis. The Air Hogs Havoc micro-helicopter emulates the Black Hornet micro reconnaissance UAV which is the smallest known military UAV. A quadcopter 
and a hexacopter are chosen to study how multiple propellers spinning at different rates affect micro-Doppler signatures. The Parrot Disco is representative of fixed wing reconnaissance drones where the sensor is in the front and the engine is in the back. Figure 10 illustrates the different UAVs used in this thesis. Table 2 lists the specifications of the drones used in this thesis.
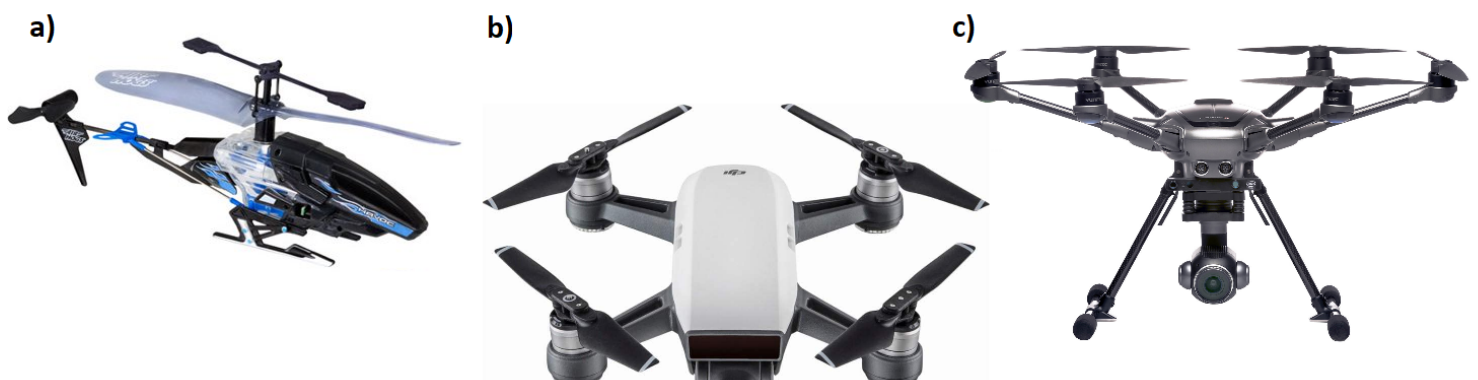

d)

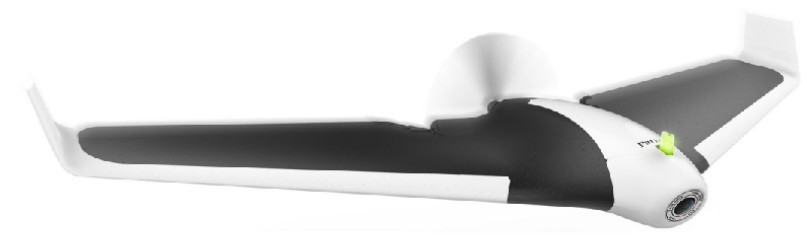

Figure 10: a) Air Hogs Havoc micro-helicopter drone b) DJI Spark quadcopter c) Typhoon $\mathrm{H}$ hexacopter d) Parrot Disco fixed wing drone.

Table 2: UAV specifications.

\begin{tabular}{|l|l|l|l|}
\hline UAV & Size $(\mathbf{L} \times \mathbf{W} \times \mathbf{H})$ & Blade Length & Type \\
\hline Air Hog Havoc & $15 \mathrm{~cm} \times 5 \mathrm{~cm} \times 7 \mathrm{~cm}$ & $6.5 \mathrm{~cm}(3.5 \mathrm{~cm})$ & Micro-helicopter \\
\hline DJI Spark & $26 \mathrm{~cm} \times 26 \mathrm{~cm} \times 5 \mathrm{~cm}$ & $6 \mathrm{~cm}$ & Quadcopter \\
\hline Typhoon H & $52 \mathrm{~cm} \times 45.7 \mathrm{~cm} \times 31 \mathrm{~cm}$ & $11 \mathrm{~cm}$ & Hexacopter \\
\hline Parrot Disco & $115 \mathrm{~cm} \times 58 \mathrm{~cm} \times 12 \mathrm{~cm}$ & $11 \mathrm{~cm}$ & Fixed Wing \\
\hline
\end{tabular}

Experiments were conducted both at Defence Research and Development Canada (DRDC) and National Research Council Canada (NRC). Experiments at DRDC were 
done using the Xethru X4 and the TI AWR1642 radar while the experiments conducted at NRC were done using the Ancortek radar. Table 3, 4 and 5 show the radar specifications of the Xethru X4, the TI AWR1642 FMCW radar and the Ancortek SDR-KIT 580 AD2 FMCW radar. Refer to the Appendix B for for more detailed information about these radars.

Table 3: AWR1642 Radar Configuration.

\begin{tabular}{|l|l|l|l|}
\hline Chirp Bandwidth & Center Frequency & Samples per chirp & Chirp duration \\
\hline $1 \mathrm{GHz}$ & $77 \mathrm{GHz}$ & 256 & $35 \mathrm{us}$ \\
\hline Max range & Range resolution & Max Velocity & $P_{t}$ \\
\hline $25 \mathrm{~m}$ & $0.142 \mathrm{~m}$ & $27.86 \mathrm{~m} / \mathrm{s}$ & $0.0177 \mathrm{~W}$ \\
\hline
\end{tabular}

Table 4: Ancortek Radar SDR-KIT 580 AD2 Configuration.

\begin{tabular}{|l|l|l|l|}
\hline Chirp Bandwidth & Center Frequency & Samples per chirp & Chirp duration \\
\hline $400 \mathrm{MHz}$ & $5.8 \mathrm{GHz}$ & 128 & $50 \mathrm{~ms}$ \\
\hline Max range & Range resolution & Max Velocity & $P_{t}$ \\
\hline $24 \mathrm{~m}$ & $0.18 \mathrm{~m}$ & $25.86 \mathrm{~m} / \mathrm{s}$ & $0.1 \mathrm{~W}$ \\
\hline
\end{tabular}

Table 5: Xethru Radar Configuration.

\begin{tabular}{|l|l|l|l|l|}
\hline PRF & Max Range & Range Resolution & Max Velocity & $P_{t}$ \\
\hline $1.5 \mathrm{KHz}$ & $10 \mathrm{~m}$ & $5.25 \mathrm{~cm}$ & $15.4 \mathrm{~m} / \mathrm{s}$ & $0.0025 \mathrm{~W}$ \\
\hline
\end{tabular}

The experiments were all conducted in an indoor lab environment with the drones fixed onto a stand a certain distance away from the radar. Figure 11 shows an example of the experimental setup at NRC with the Ancortek radar and the Typhoon $\mathrm{H}$ hexacopter.

For each drone, two experiments were conducted, one with the propellers spinning at idle to emulate a hovering scenario and one with propellers spinning at varying 


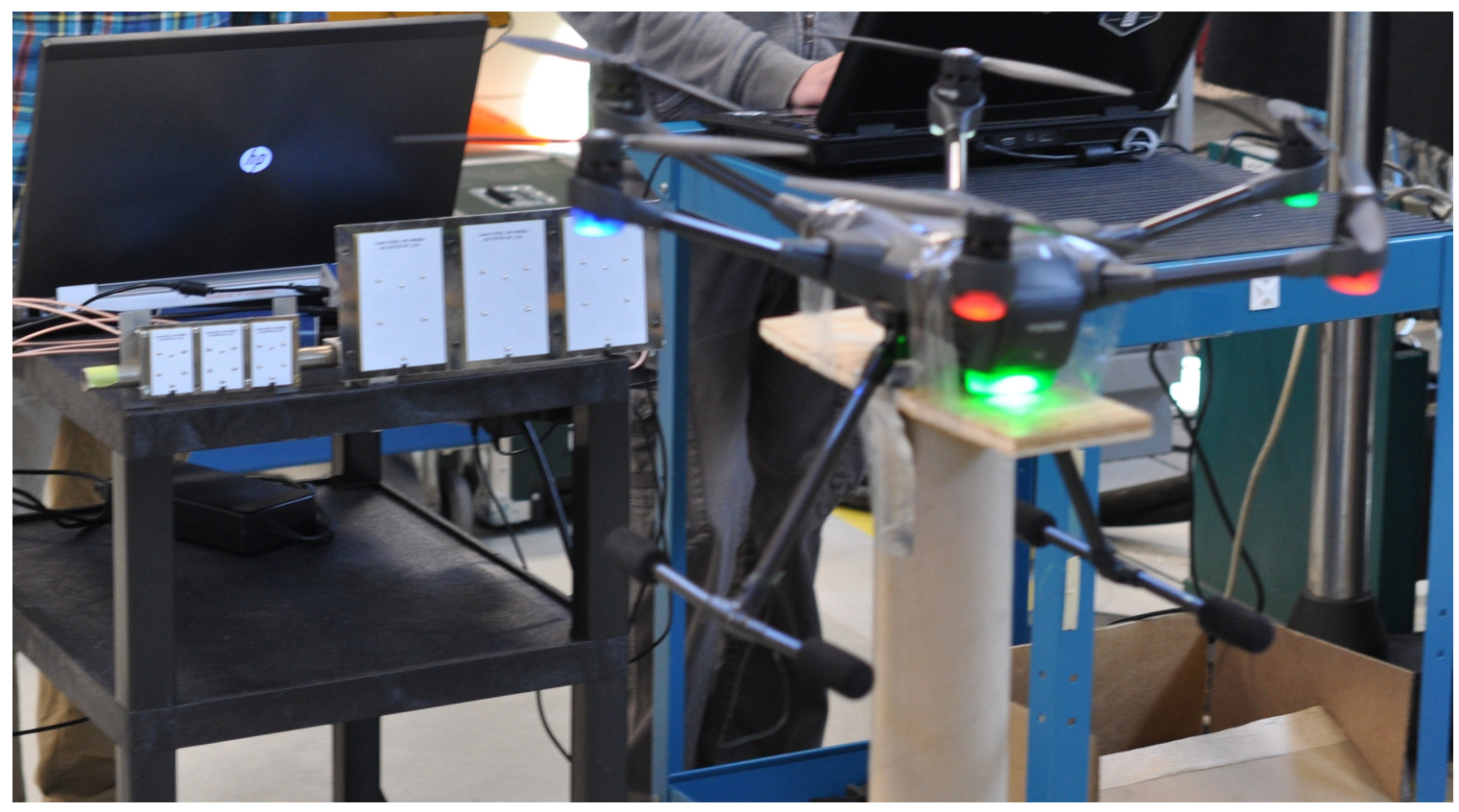

Figure 11: Experimental setup for drone trials at NRC.

speeds to study a maneuvering scenario. Flying experiments were done exclusively for the DJI Spark. In addition to this, the micro-Doppler signature of a waving hand was used to emulate bird micro-Doppler.

\subsection{Experimental Results}

\subsubsection{Air Hogs Havoc Micro-helicopter}

The first UAV examined is a simple two bladed single-rotor Air Hog Havoc microhelicopter. Figure 12 shows the short-window STFT signature obtained from the TI radar on the left and the HERM line signature from the Xethru X4 radar on the right. Both signatures follow a downward sloping pattern as the motor decelerated 
to steady state.
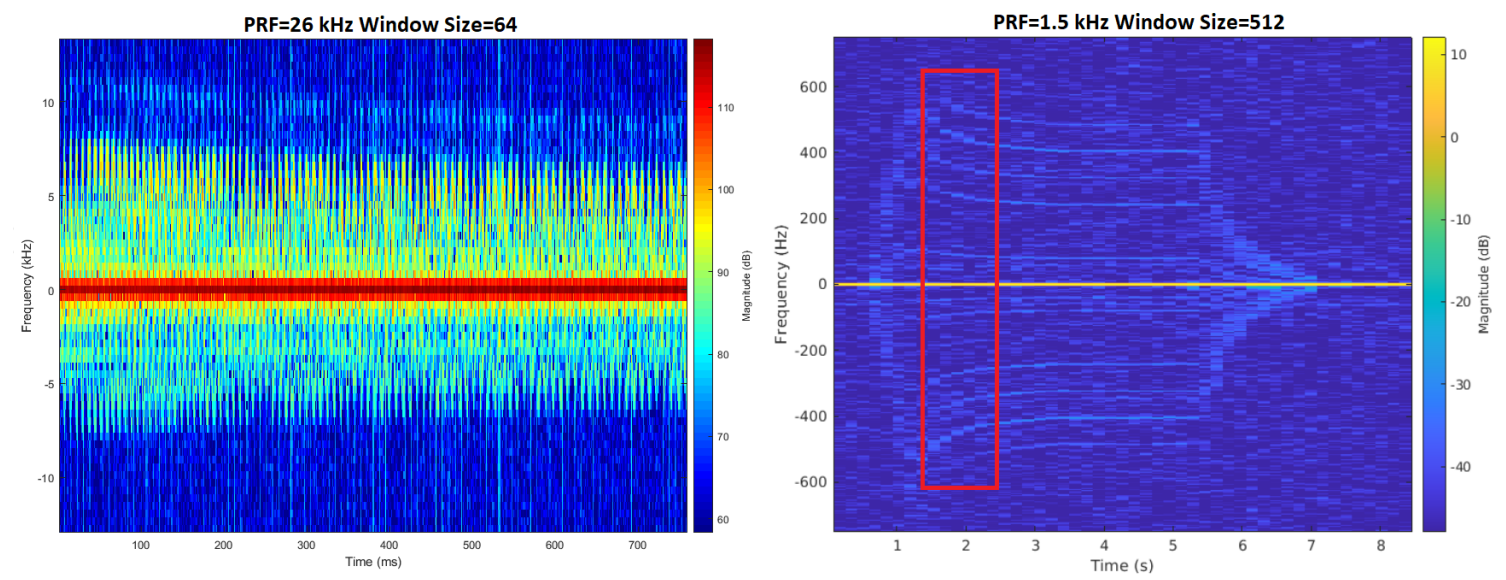

Figure 12: Micro-Doppler signature captured by the TI radar on the left and HERM line signature from the Xethru radar on the right.

The rotation rate can be estimated from both short-window and long-window spectrum. For the HERM line signature, the rotation rate can be found by dividing the HERM line separation frequency by the number of blades [10]. The separation frequency at steady state was found to be $80 \mathrm{~Hz}$ and therefore, the rotation rate is calculated as $40 \mathrm{r} / \mathrm{s}$ for this two bladed UAV. For the short window STFT signature, the rotation rate can be found by measuring the time difference between the blade flashes. As shown in Figure 13, this periodicity is about $26 \mathrm{~ms}$ which means the rotation rate is about $1 / 26 \mathrm{~ms}=38 \mathrm{r} / \mathrm{s}$. This is consistent with the estimate from the HERM line signature.

The blade length can be estimated using the periodicity and the maximum induced micro-Doppler using the following equation [15]:

$$
l=\frac{V_{t i p}}{2 \pi \Omega}
$$

The micro-Doppler shift induced by the rotating propeller is $8.6 \mathrm{KHz}$ which corresponds to a blade tip velocity of $16.75 \mathrm{~m} / \mathrm{s}$ for a radar carrier frequency of $77 \mathrm{GHz}$. 


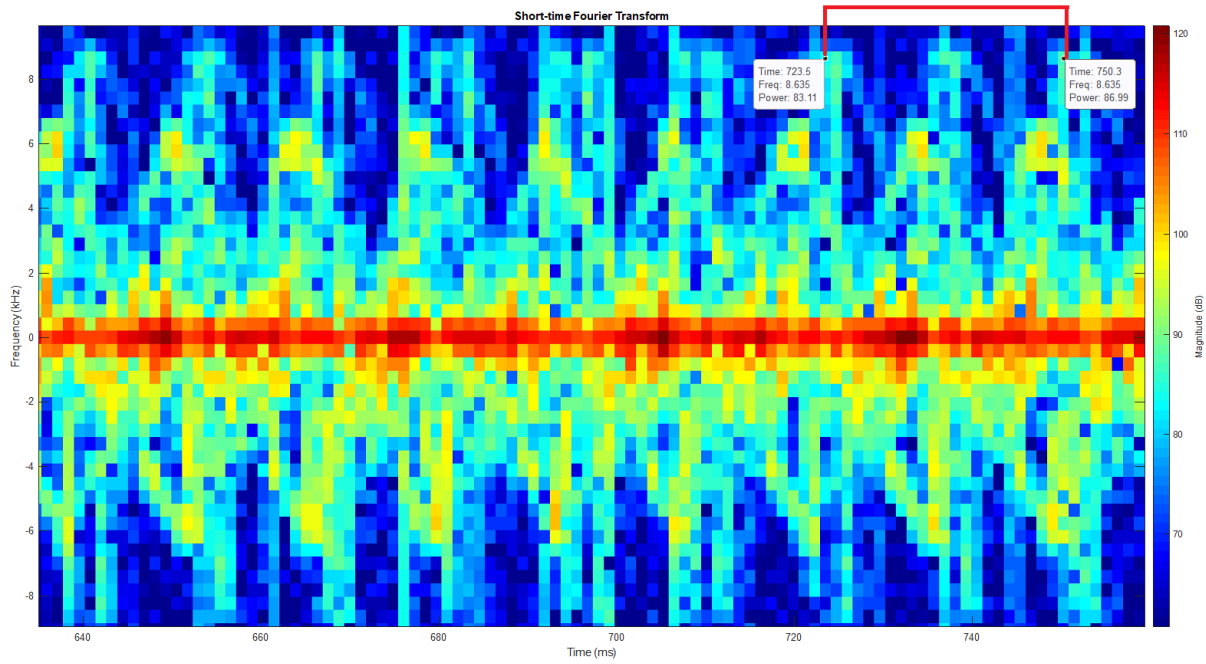

Figure 13: Zoomed in micro-Doppler spectrogram of micro-helicopter drone at steady state.

This corresponds to be blade length of $6.6 \mathrm{~cm}$ for a propeller rotation rate of $40 \mathrm{r} / \mathrm{s}$. This is consistent with the true blade length of $6.5 \mathrm{~cm}$.

The blade length cannot be estimated with the Xethru X4 radar since the tip velocity of $16.75 \mathrm{~m} / \mathrm{s}$ exceeds the maximum unambiguous velocity of $15 \mathrm{~m} / \mathrm{s}$. This is because the PRF is not high enough, as is typically the case for many practical radars.

\subsubsection{Multi-rotor Drones}

Multi-rotor drones are the most common off-the-shelf drones that civilians can purchase. As such, they are responsible for many drone-related incidents. In this study, two different multi-rotor drones were examined, the DJI Spark mini quadcopter and the Typhoon $\mathrm{H}$ hexacopter. These drones were operating at idle conditions fixed on a stand, all the rotors were spinning at the same rate similar to a single rotor 
drone. However, when the speeds of the rotors were varied, this introduced additional fundamental frequencies to the HERM line spectrum. This is shown in Figure 14 for the Typhoon $\mathrm{H}$ hexacopter fixed on stand $2 \mathrm{~m}$ away from the radar. After 5 seconds, the HERM lines split up into multiple trajectories due to the different rotor speeds. The HERM lines are not constantly spaced anymore. This is one feature which distinguishes single rotor drones from multi-rotor drones.

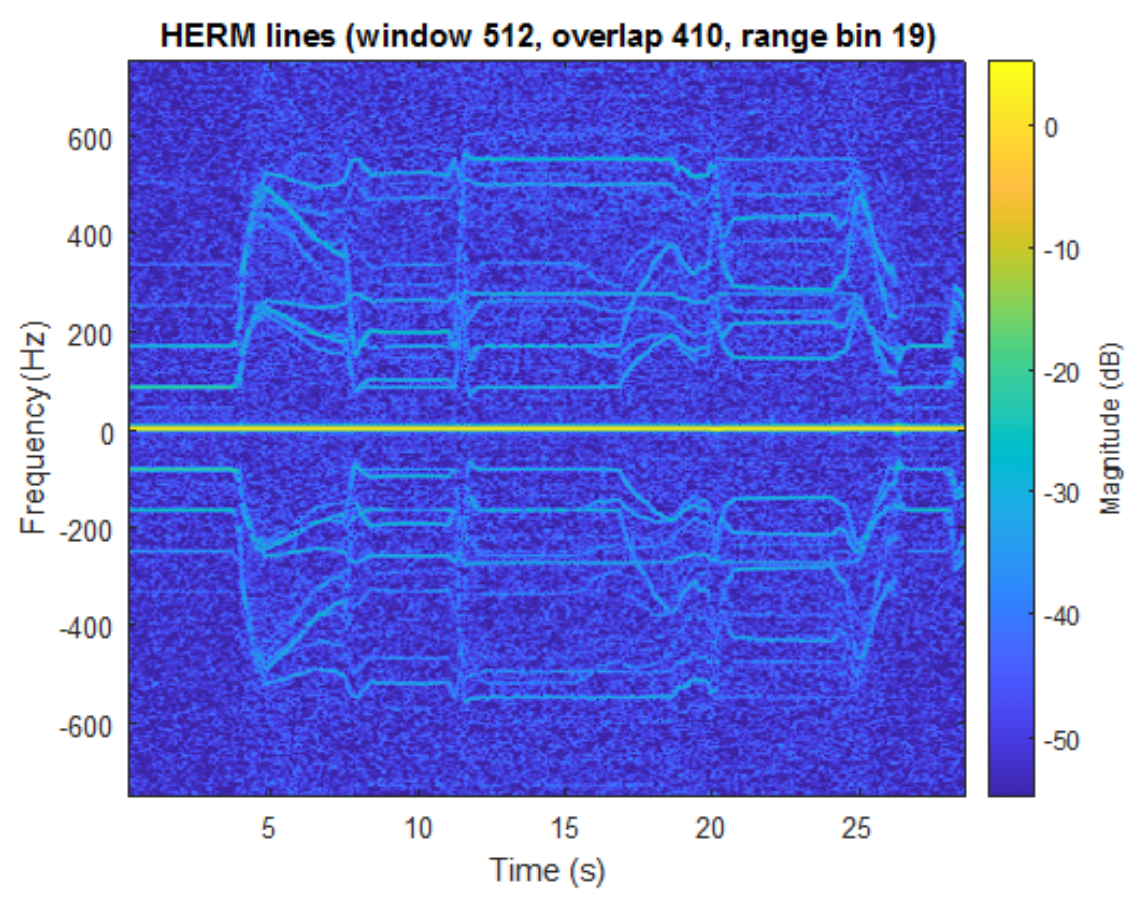

Figure 14: HERM line signature of hexacopter on a stand with varying rotor speeds.

Flying experiments were conducted with the DJI Spark at NRC using the Ancortek radar. It was found that even when the drone was hovering in an indoor lab environment, the rotors were not spinning at exactly the same rate. This is shown by the very small frequency deviations between the HERM lines in Figure 15. The large fundamental frequency differences when the drone was on a stand (Figure 14) is not seen for a real flying scenario. Detecting these small frequency deviations may be a way of distinguishing a multi-rotor drone from a single rotor drone. 


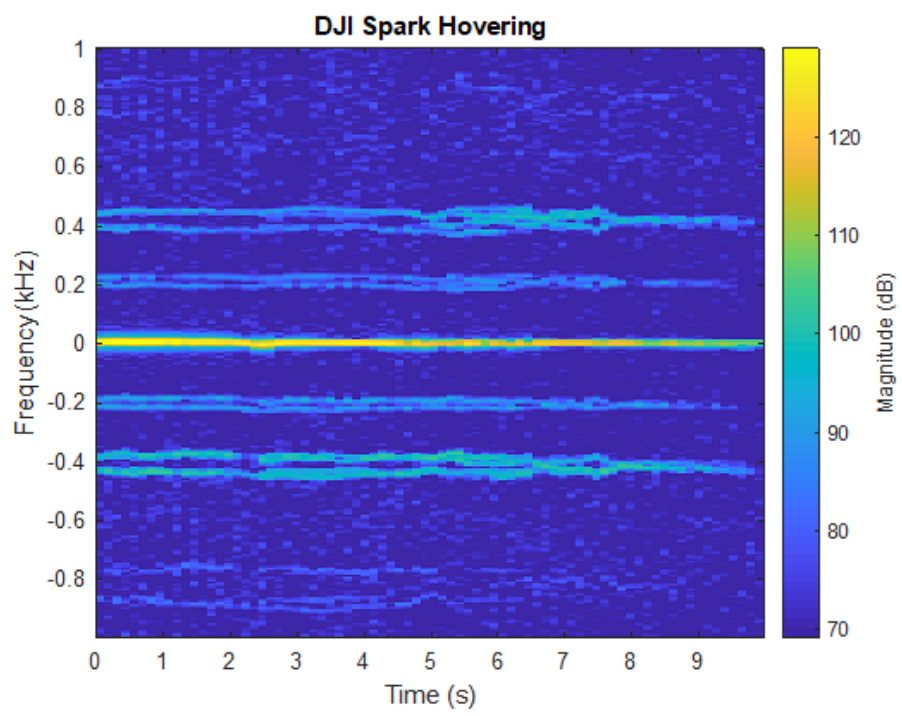

Figure 15: HERM line signature of hovering DJI Spark $4 \mathrm{~m}$ away from the Ancortex radar.

Figure 16 shows an example of the short-window STFT micro-Doppler signature of a hexacopter in comparison to a single rotor drone obtained using the TI AWR1642 radar. It is much harder to visually identify the target as a multi-rotor drone using the short-window STFT representation. This is despite the fact that the PRF is adequate. This demonstrates the advantage of using the HERM line representation.

\subsubsection{Parrot Disco Fixed Wing Drone}

The Parrot Disco is a large fixed wing drone with a camera sensor in the front and a single propeller in the back. Its design is very popular for military reconnaissance drones since it allows a full unobscured view for the sensors in the front. This design also provides micro-Doppler shielding since the propellers are hidden by the fuselage and the large wing. Figure 17 shows the HERM line spectrogram of the Parrot drone captured from the side and front of the drone at $1 \mathrm{~m}$. The micro-Doppler 

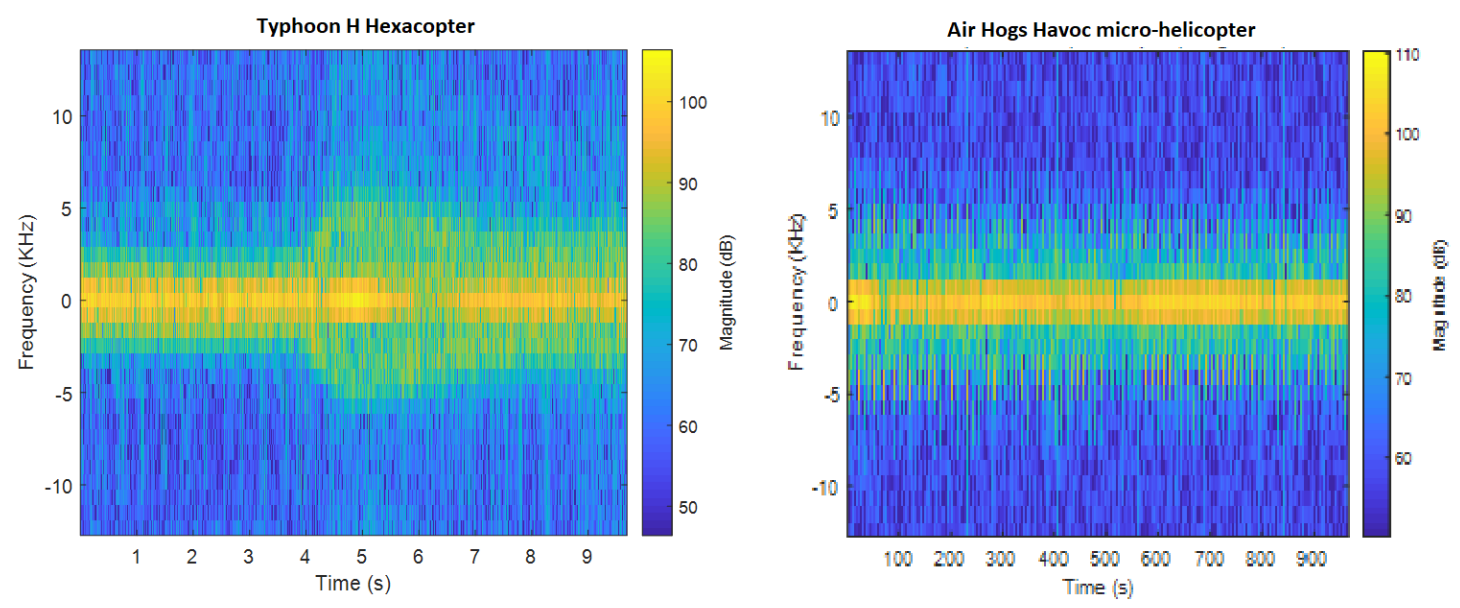

Figure 16: Short windowed STFT micro-Doppler signatures of multi-rotor and single rotor drones.

signature is stronger when captured from the side than from the front of the drone. This could pose a problem for feature extraction if the drone is flying towards the radar. The HERM line separation frequency is very high at about $375 \mathrm{~Hz}$. This is larger than all of the other drones studied. Fixed wing drones will typically have higher propeller rotation rates than rotary drones since they fly faster and higher. Distinguishing between rotary and fixed wing drones is important for threat analysis. Most commercial consumer drones are quadcopters while military drones are usually fixed wing for faster and long range flight. The HERM line separation frequency could be a potential feature to distinguish between these two types of drones.

\subsubsection{Birds}

Birds are often mistaken for drones since they have similar RCS and fly at relatively the same speeds and altitude. Birds also induce periodic micro-Doppler signatures due to their flapping wings. However, birds typically flap their wings between $3-20 \mathrm{~Hz}$ (3-20 times a second) and also go through periods of time without flapping (gliding). 

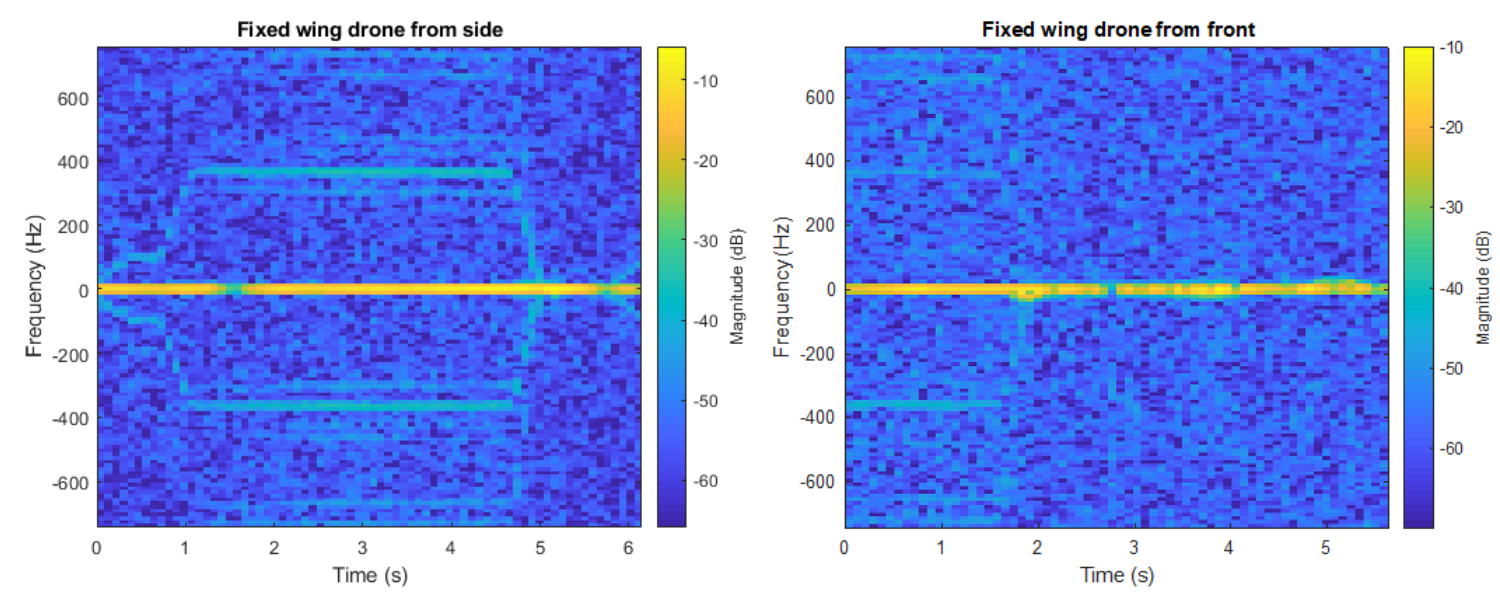

Figure 17: STFT spectrogram of the fixed wing drone captured with the Xethru X4 radar showing HERM lines.

Flying drones have much higher propeller rotation rates of 100 to $300 \mathrm{~Hz}$ and have to constantly spin their propellers to stay in the air [10]. The micro-Doppler signature of a waving hand is used to emulate the micro-Doppler signature of a bird. Figure 18 shows the STFT spectrogram of a waving hand at $3 \mathrm{~Hz}$ generated using a window period of $0.064 \mathrm{~s}$ (128 samples) in comparison with a helicopter drone captured using the Ancortek radar.

Since the waving hand motion is only at $3 \mathrm{~Hz}$, a window period of $0.064 \mathrm{~s}$ is only a fraction of the period so a wideband spectrogram is produced. On the other hand, the window period of 0.064 s covers multiple rotation periods of the drone so a HERM line signature is produced. Thus, for a fixed window period of more than 1/100 and less than 1/30, the STFT spectrogram will be narrowband for a drone but not for a bird. This phenomenon is also shown for real bird STFT micro-Doppler signatures [31]. 

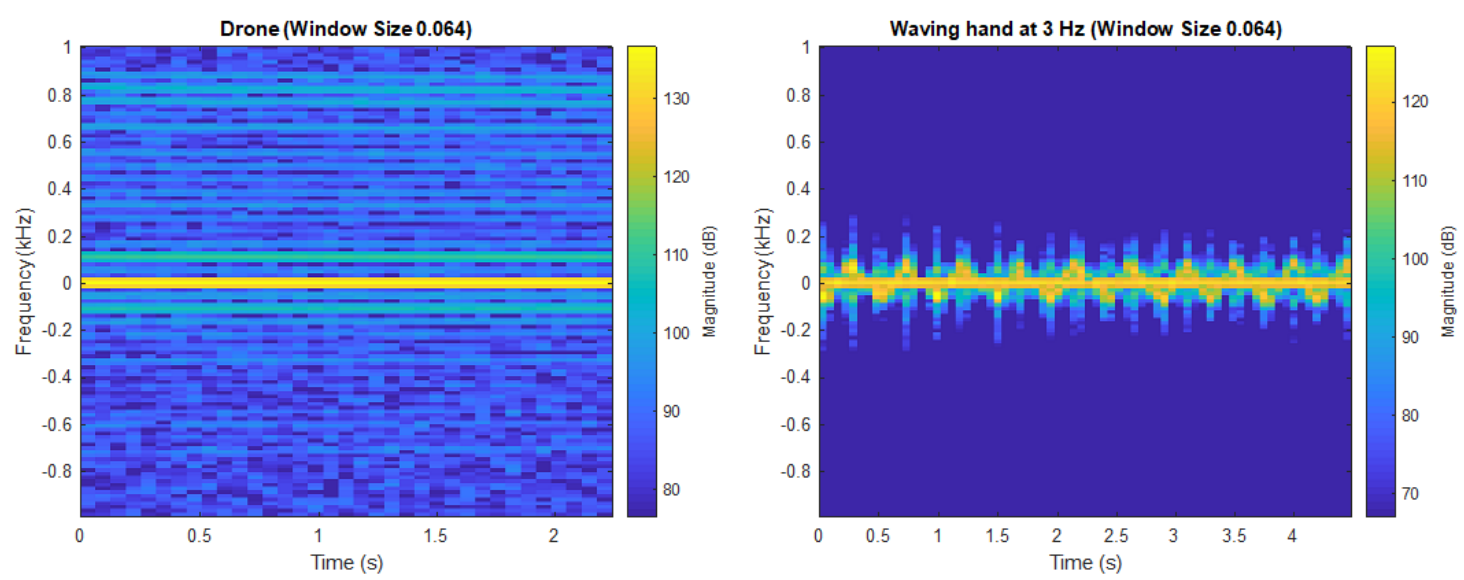

Figure 18: STFT spectrogram of a waving hand and a micro-helicopter drone using the same window period of $0.064 \mathrm{~s}$.

\subsection{Time Series Model of Rotor Micro-Doppler}

\subsubsection{Time Series Model}

Based on the above experimental results, a simple model for HERM line signatures is presented. This forms the basis for all the HERM line processing techniques in the subsequent chapters. Although other models for the micro-Doppler of a spinning propeller exist [15], [16], the proposed model is simpler and representative of the experimental results. Since the HERM line signature of a single rotating propeller just consists of $M$ spectral lines separated in frequency by $f \mathrm{~Hz}$, this can be represented by a sum of $M$ sinusoids with fundamental frequency $f \mathrm{~Hz}$.

$$
S[n]=a_{0}+\sum_{m=1}^{M} a_{m} \sin \left[2 \pi m f n+\phi_{m}\right]+n_{0}[n],
$$

The first term $a_{0}$ represents the amplitude of the main Doppler component and the subsequent terms represent the $M$ HERM lines that repeat at separation frequency $f$, and $n_{0}(t)$ is the Additive White Gaussian Noise (AWGN) added to simulate real 
life circumstances. It is found in the literature that the strength of micro-Doppler components are typically 20-40 dB lower than the body line [32]. The total number of HERM line components $M$ on one side is related to the maximum Doppler shift induced by the propeller and the blade length [16],

$$
M=\frac{f_{D \max }}{N \Omega}
$$

where $f_{\text {Dmax }}$ is the maximum Doppler shift induced by the rotating propeller and is given by

$$
f_{D \max }=\frac{4 \pi l \Omega \cos (\theta)}{\lambda} .
$$

Here, $N$ is the number of blades and $\Omega$ is the rotation rate, $\lambda$ is the operating wavelength of the radar, $l$ is the length of the propeller blade, and $\theta$ is the angle between the plane of rotation and the line of sight. This can be simplified to the following expression.

$$
M=\frac{4 \pi l \cos (\theta)}{N \lambda} .
$$

Note that the total number of HERM lines for a two-sided spectrum is $2 M$. In the case of a low PRF radar, like the Xethru X4, the total number of HERM line components on one side will be less than $M$ since it will not be able to capture the full micro-Doppler shift of the spinning propeller.

Using this model, one can generate micro-Doppler signatures of propellers spinning at different rates with different blade lengths. Figure 19 shows an example of a simulated micro-Doppler signature of a $4 \mathrm{~cm}$ long two bladed propeller spinning at 25 $\mathrm{Hz}$ sensed with a radar whose PRF is $1.5 \mathrm{KHz}$. The maximum tip velocity would be $2 \pi l \Omega=6.15 \mathrm{~m} / \mathrm{s}$. This corresponds to a maximum Doppler shift of $300 \mathrm{~Hz}$. Since the two bladed propeller is spinning at $25 \mathrm{~Hz}$, the separation frequency is $50 \mathrm{~Hz}$. There will be 6 HERM lines from 50 to $300 \mathrm{~Hz}$. The model is written as:

$$
S[n]=a_{0}+\sum_{m=1}^{6} a_{m} \sin [2 \pi m 50 n]+n_{0}[n],
$$

The amplitude of the HERM line components $\left(a_{m}\right)$ are set to 0.1 and $a_{0}=1$ so that they are $20 \mathrm{~dB}$ (in power) lower than the body line. 


\subsubsection{Short-Window and Long-Window STFT}

Figure 19 shows an example of a short and long windowed STFT spectrogram generated using Equation 32. Using a short window of 64 from a radar with PRF of 1.5 $\mathrm{KHz}$ (approximately $0.04 \mathrm{~s}$ ), the blade flash phenomenon is seen.
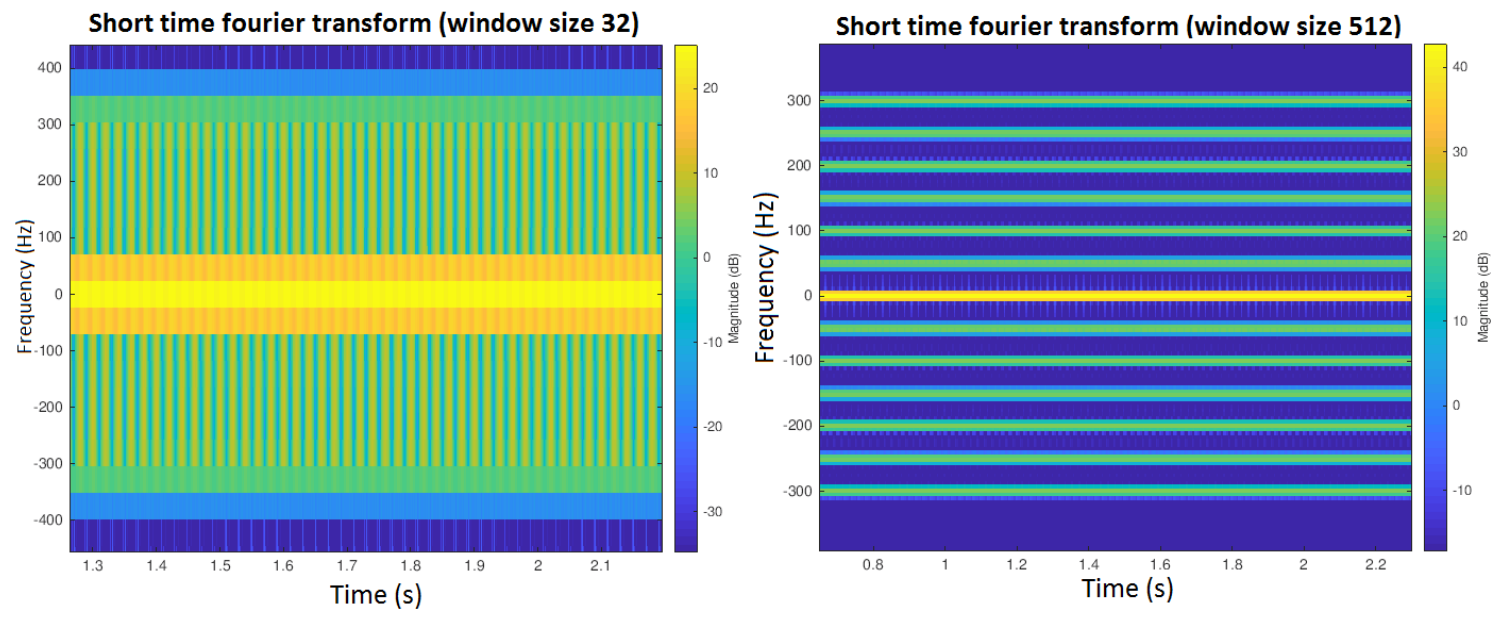

Figure 19: STFT of HERM lines generated using the HERM line model.

For a multi-rotor drone, the micro-Doppler signature becomes a superposition of the radar backscatter from all the spinning propellers. Each unique spinning propeller will produce its own fundamental frequency $f_{c}$. Thus, the mathematical model for the micro-Doppler signature of a multi-rotor drone becomes

$$
S[n]=a_{0}+\sum_{c=1}^{C} \sum_{m=1}^{M} a_{c m} \sin \left[2 \pi f_{c} m n+\phi_{c m}\right]+n_{0}[n],
$$

where $C$ is the number of fundamental frequencies. From experimental results, it was found that each rotor generates the same number of HERM lines (see Figure 14 and 15). Typically for multi-rotor drones, pairs of propellers will change speeds at a time to maintain balance. This means that for a quadcopter, there would be two unique fundamental frequencies $(C=2)$. The number of unique fundamental frequencies could be used to distinguish between single and multi-rotor drones. 


\subsubsection{Comparison with Martin-Mulgrew Models}

A well known mathematical model of the radar backscatter of a single aircraft propeller is by Martin-Mulgrew [16] which uses six variables, four of which are related to the propeller, one related to the radar signal strength and one related to the aspect angle of the propeller. This model treats the propeller as a flat rotating antenna with $L_{1}$ being the length of the propeller hub to the center and $L_{2}$ being the length from the blade tip to the center. This is shown in Figure 20.

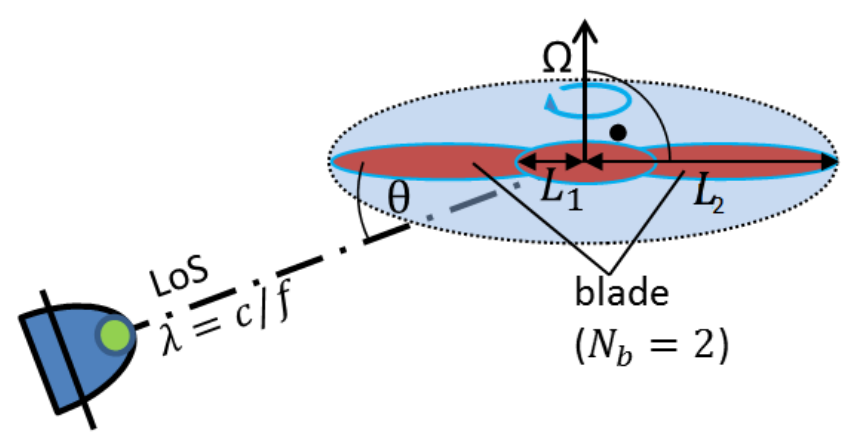

Figure 20: Diagram of rotating propeller with respect to the radar line of sight.

$$
\begin{aligned}
v_{r}(t)= & \sum_{n=0}^{N-1} A_{r}\left(L_{2}-L_{1}\right) e^{j\left(\omega_{c} t-\frac{4 \pi}{\lambda}\left(R+v t+\frac{\left(L_{1}+L_{2}\right)}{2} \cos (\theta) \sin \left(\omega_{r} t+\frac{2 \pi n}{N}\right)\right)\right)} \\
& \operatorname{sinc}\left(\frac{4 \pi}{\lambda} \frac{\left(L_{2}-L_{1}\right)}{2} \cos (\theta) \sin \left(\omega_{r} t+\frac{2 \pi n}{N}\right)\right)
\end{aligned}
$$


where

$$
\begin{aligned}
A_{r} & =\text { scale factor related to radar power } \\
L_{1} & =\text { length from blade root to center } \\
L_{2} & =\text { length from blade tip to center } \\
N & =\text { number of blades } \\
R & =\text { range to center of rotation } \\
t & =\text { time } \\
v & =\text { radial velocity of the center of rotation with respect to the radar } \\
\lambda & =\text { wavelength } \\
w_{c} & =\text { radian frequency of the transmitted wave } \\
\theta & =\text { aspect angle between the plane of rotation and the line of sight of the radar } \\
w_{r} & =\text { radian frequency of propeller rotation }
\end{aligned}
$$

In comparison, the continuous time equivalent of the proposed micro-Doppler model for a single rotor drone is

$$
S(t)=a_{0}+\sum_{m=1}^{M} a_{m} \sin \left(2 \pi m F t+\phi_{m}\right)+n_{0}(t)
$$

where $M$ is

$$
M=\frac{4 \pi L_{2} \cos (\theta)}{N \lambda} .
$$

and $F$ is analog equivalent fundamental frequency of the HERM lines.

Since the size of the propeller hub for a drone is negligible, $L_{1}$ is ignored. Taking the Fourier transform of the Martin-Mulgrew model also results in spectral peaks with frequency separation spacing $N \Omega$. However, the amplitudes of the spectral peaks are more complicated and is represented by

$$
V_{r}(f)=\sum_{k=-M}^{M} c_{N k} \delta\left(f-f_{d}-N k \Omega\right)
$$


where

$$
\begin{aligned}
c_{N k} & =\sum_{l=0}^{\infty} \frac{2(-1)^{N k u(k)} A_{r} N}{4 \pi / \lambda \cos (\theta)}\left(J_{|N k|+2 l+1}\left(4 \pi / \lambda\left(L_{2}-L_{1}\right) \cos (\theta)\right)\right) e^{-j 4 \pi R / \lambda}, \\
J_{k} & =\text { Bessel function of first kind and kth order } \\
f_{d} & =\text { Doppler shift of body } \\
u(k) & =\text { unit step function }
\end{aligned}
$$

(Note that $f$ here represents the continuous Fourier frequency. This should not be confused with the notation reuse as fundamental frequency of HERM lines).

In contrast, the amplitudes of the HERM line components in Equation 28, $\left(a_{m}\right)$, are set to a constant value, -20 to $-40 \mathrm{~dB}$ lower than $a_{0}$. In this thesis, only frequencybased features are considered. Therefore accurate modeling of the amplitudes is not necessary.

In 1992, Martin-Mulgrew published an updated model for a rotating propeller which takes into consideration the pitch of the blades [33]. When the propeller blades are pitched and the aspect angle $\theta$ is positive (tilting towards the radar), the cross-section area of the blade will be greater when the blade is rotating away from the radar than when the blade is rotating towards the radar. This causes an asymmetry in the microDoppler spectrum where the magnitudes of the sidebands in the negative Doppler are greater than the positive Doppler. The opposite is seen when the aspect angle is negative (tilting away from the radar). If $\theta$ or the pitch of the blades is large enough, the sidebands at one half of the spectrum could completely disappear. In this thesis, all the experiments were conducted with the radar in line of sight with the drone $\left(\theta=0^{\circ}\right)$ so the aspect angle dependence was not observed.

The proposed time series models (Equation 28 and Equation 33) in this thesis reproduces the spectrum of Equation 37 and is considerably simpler than the MartinMulgrew model. Furthermore, the proposed model is amenable to several standard spectral estimation techniques. However, this comes at a cost. In particular, the 
connection to radar parameters and aspect angle dependence is lost.

\subsection{Conclusions}

From these experiments, several HERM line features were identified to classify between birds, single rotor and multi-rotor drones.

- The HERM line separation frequency is related to the propeller rotation rate. Fixed wing drones were found to have larger HERM line separation frequency than rotary drones since they cannot hover. The following table can be used as a reference for classifying different drones.

Table 6: HERM line separation frequency for different drones.

\begin{tabular}{|l|l|}
\hline Drone Type & HERM line separation \\
\hline Multi-rotor & $100-260 \mathrm{~Hz}$ \\
\hline Fixed Wing & $200-500 \mathrm{~Hz}$ \\
\hline Helicopter & $50-80 \mathrm{~Hz}$ \\
\hline
\end{tabular}

- Multi-rotor drones have multiple fundamental frequencies induced by different rotor speeds. For basic maneuvers, multi-rotor drones usually change the speeds of pairs of propellers at a time so the number of unique fundamental frequencies is equal to the total number of rotors divided by 2 . The number of unique fundamental frequencies can be used to distinguish single and multi-rotor drones.

- Given that the STFT window size is selected to be less than the wing flap period of a bird but more than the propeller rotation period of a drone, different spectrogram representations will be generated for the birds and drones. The spectrogram for the drone will be narrowband while the bird spectrogram will be wideband. This could be used to distinguish between birds and drones. 
In addition, a simple mathematical model was created to represent the micro-Doppler signal obtained from single rotor and multi-rotor drones. This model shows that the micro-Doppler signal from a UAV can be simplified into three variables: the number of HERM lines $M$ per propeller, the rotor rotation rates $f_{c}$ and the number of unique rotation rates $C$. However, in practice, the rotor rotation rates $f_{c}$ for a multi-rotor drone are nearly identical which makes estimation of $C$ difficult. The following chapters will focus on developing signal processing techniques to extract two of these features, the total number of HERM lines $(M \times C)$ and the fundamental frequency, $f_{c}$. 


\section{Chapter 4}

\section{Parametric Estimation of the Number of HERM lines}

A defining feature of a HERM line signature is the number of HERM lines $(M \times C)$. The number of HERM lines is a function of the maximum micro-Doppler shift and the propeller rotation rate. Fioranelli et al [6] used the number of HERM lines to distinguish between drones and clutter based on peak detection in the HERM line spectrogram. However, this method may not work well under low SNR conditions as it becomes difficult to discern which peaks correspond to noise and which peaks correspond to signal. Fourier-based methods also suffer from poor resolution for small sample sizes. In this chapter, the number of HERM lines was estimated using techniques based on the ordered eigenvalues of the signal covariance matrix. Popular techniques in this area of research were applied to compare the performance.

Based on these results, a rotary wing drone detector was proposed using Minimum Description Length (MDL). This method does not require any prior information about the radar target and only requires a short amount of data. 


\subsection{Introduction}

A method of studying the frequency content of micro-Doppler without using the Fourier transform is based on Singular Spectral Analysis (SSA). SSA decomposes a time series into a sum of signal and noise components using the eigendecomposition of the covariance matrix. Clemente [34] demonstrated that SSA can decompose the micro-Doppler signature of a helicopter into the body line, micro-Doppler and noise components. The reconstructed time series using the first principal component corresponds to the main body while using the subsequent components reveal the micro-Doppler blade flashes.

Another feature that can be extracted from eigendecomposition of the covariance matrix is the subspace dimension, or the number of components in the signal subspace. Given a discrete time series $x[n]$ comprised of $M$ sinusoids in white noise

$$
x[n]=\sum_{m=1}^{M} a_{m} \sin \left[2 \pi f m n+\phi_{m}\right]+n_{0}[n],
$$

where $a_{m}, f$ are the amplitudes and frequencies of the harmonics and $n_{0}[n]$ is independent white noise with variance $\sigma^{2}$. Assuming that the noise is zero mean and independent of the signal, the covariance matrix of a segment of $\mathbf{x}$, i.e., $y[n]=$ $[x[n], \ldots, x[n-N+1]]$ denoted $y[n]$ can be written as [35]:

$$
\begin{aligned}
R & =\mathbf{E}\left[\mathbf{x x}^{\mathbf{T}}\right], \\
& =\Psi+\sigma^{2} I,
\end{aligned}
$$

where

$$
\Psi=A S A^{T}
$$

where $\mathbf{E}$ is the expectation operator, $T$ is the transposition operator. For the noiseless case, the eigenvalues of $\Psi$ will contain $M$ non-zero values and in white noise, there will be $N-M$ eigenvalues equal to noise variance. 
Note that this signal model is the same as the micro-Doppler model of a single propeller defined in the previous chapter with the exception of the main Doppler component $a_{0}$. Thus, this technique could potentially be used to estimate the number of HERM line components.

In practice, the covariance matrix is estimated using finite samples. Many techniques have been developed to estimate the signal subspace dimension, or the number of harmonic components, from the covariance matrix. In this chapter, we used some well known parametric spectrum estimation methods and tested their applicability for estimating the number of HERM lines.

For each of the following methods, a covariance matrix is estimated by first constructing a trajectory matrix $\mathbf{Y}$ using $L$ lag shifted version of the radar time series $\mathbf{x}$ [34]. Each column of $\mathbf{Y}$ is a lag shifted version of $\mathbf{x}$ of length $N-L+1$. Thus, $\mathbf{Y}$ is a $(N-L+1) \times L$ matrix. Here, $\mathbf{x}$ is assumed to be wide-sense stationary for small time intervals (say over $L$ lags). The data can be either real or complex. In order to make the discussion more general, it is assumed that the data is complex.

$$
Y=\left[\begin{array}{cccc}
x[1] & x[2] & \cdots & x[N-L+1] \\
x[2] & x[3] & \cdots & x[N-L+2] \\
\vdots & \vdots & \ddots & \vdots \\
x[L] & x[L+1] & \cdots & x[N]
\end{array}\right]
$$

The covariance matrix is then computed using

$$
\mathbf{C}=\frac{1}{(N-L+1)} \mathbf{Y Y}^{\mathbf{H}}
$$

where $\mathbf{Y}^{\mathbf{H}}$ is the hermitian transposed version of $\mathbf{Y}$. The resulting covariance matrix, C will be $L \times L$, with window size $L$, limiting the maximum amount of detectable harmonics components. Also note that $N-L+1$ is also referred to as number of snapshots. 


\subsection{Parametric Spectral Estimation Techniques}

\subsubsection{Minimum Description Length (MDL) \& Aikaike Infor- mation Criterion (AIC)}

Minimum Description Length (MDL) and Aikaike Information Criteria (AIC) are information theoretic model selection methods of determining how well a family of models with parameterized probability densities $f(x \mid \theta)$ fits a dataset $X$. Both methods are based on minimizing the negative log likelihood of density $f(X \mid \theta)$ where $\theta$ is a vector of parameters for the model. The Aikaike Information Criterion is defined as

$$
\mathrm{AIC}=-2 \ln (f(X \mid \hat{\theta})+2 k
$$

where $\hat{\theta}$ is the maximum likehood estimate of parameter vector $\theta$ and $k$ is the number of adjustable parameters in $\theta[36]$.

The penalizing term $2 k$ is required because minimizing the negative log likelihood will always tend to the model with the highest order. This criterion was derived using the Kullback-Leibler mean information distance which is a measure of information loss. MDL is a method derived by Schwartz and Rissanen using Bayesian techniques which selects the model with the minimum code length [37][38].

$$
\mathrm{MDL}=-\ln \left(f(X \mid \hat{\theta})+\frac{1}{2 \ln (N)} k .\right.
$$

where $N$ is the length of the dataset. The difference between these two methods is the penalization term. AIC is known to overestimate the model order which MDL overcomes with the extra $1 / 2 \ln (N)$ factor.

These methods can be used to estimate the number of harmonic components by parameterizing the covariance matrix $R$ and finding the rank of the covariance matrix which best fits the data. The resulting two criterion were derived by Wax as [35] is 
given by

$$
\begin{gathered}
A I C(k)=-2 p \ln \left[\frac{\prod_{i=k+1}^{L} \lambda_{i}}{\left(\frac{1}{L-k} \sum_{i=k+1}^{L} \lambda_{i}\right)^{L-k}}\right]+2 k(2 L-k), \\
M D L(k)=-p \ln \left[\frac{\prod_{i=k+1}^{L} \lambda_{i}}{\left(\frac{1}{L-k} \sum_{i=k+1}^{L} \lambda_{i}\right)^{L-k}}\right]+\frac{1}{2} k(2 L-k) \ln (p) .
\end{gathered}
$$

where $k$ is the model order from 0 to $L-1, L$ is the number of eigenvalues of the sample covariance matrix, $\lambda$ are the eigenvalues of the sample covariance matrix ordered from greatest to least, $N$ is the total number of samples and $p=N-L+1$. The model order $k$ which minimizes the criterion is chosen as the number of sources. When using AIC, the number of sources is selected as

$$
n=\min _{k} A I C(k)
$$

and while using MDL, the number of source is obtained as follows:

$$
n=\min _{k} M D L(k)
$$

\subsubsection{Radoi Method}

The Radoi method is an empirical method for estimating the signal subspace.It has the interesting property of overestimating the number of harmonic components at low SNR scenarios. This is useful since the HERM line components are much weaker than the main Doppler component so underestimation of the number of HERM line components is expected at low SNR. This method should perform better than classical methods such as MDL and AIC under low SNR.

For this method, two discriminate functions are defined: $g_{1}(k)$ representing the probability density function that the corresponding eigenvalue belongs to the signal subspace and $g_{2}(k)$ representing the probability density function that the corresponding eigenvalue belongs to the noise subspace. $g_{1}(k)$ is derived as the normalized values of 
the last $L-1$ eigenvalues ordered from greatest to least.

$$
g_{1}(k)=\frac{\lambda_{k+1}}{\sum_{i=2}^{L} \lambda_{i}}, \quad k=1, \ldots, L-1,
$$

where $\lambda_{k}$ are the ordered eigenvalues from 1 to $L$. The probability density function corresponding to the noise subspace, $g_{2}(k)$, has an inverse relationship to $g_{1}(k)$ and is found by examining the slope variation of the ordered eigenvalues.

$$
g_{2}(k)=\frac{\xi_{k}}{\sum_{i=1}^{L-1} \xi_{i}}, \quad k=1, \ldots, L-1,
$$

where

$$
\begin{aligned}
\xi_{k} & =1-\alpha\left(\lambda_{k}-\mu_{k}\right) / \mu_{k}, \\
\mu_{k} & =\frac{1}{(L-k)} \sum_{i=k+1}^{L} \lambda_{i},
\end{aligned}
$$

and

$$
\alpha=\frac{1}{\max _{k} \frac{\lambda_{k}-\mu_{k}}{\mu_{k}}} .
$$

This definition of $\alpha$ ensures that $g_{2}(k)$ is not negative. The number of harmonic components is then estimated by finding $k$ such that the cost function $C(k)=g_{1}(k)-$ $g_{2}(k)$ is minimized.

\subsection{Performance Evaluation using Time Series Micro- Doppler Model}

\subsubsection{Single Rotor Drone}

To test the performance of these parametric spectral estimation methods, a HERM line signature was modelled using the simple time series model defined in Equation 
28 with fundamental frequency of $100 \mathrm{~Hz}$ and a DC component $a_{0}$. The model parameters used are listed in the table below.

Table 7: Single Rotor HERM line model parameters.

\begin{tabular}{|l|l|}
\hline Parameter & Value \\
\hline$a_{0}$ & 1 \\
\hline$a_{n}$ & 0.1 \\
\hline$f_{c}$ & 100 \\
\hline$M$ & 2 \\
\hline $\mathrm{PRF}$ & $1.5 \mathrm{KHz}$ \\
\hline
\end{tabular}

The sinusoidal components are set to be $20 \mathrm{~dB}$ lower than the $\mathrm{DC}$ component $a_{0}$ since the micro-Doppler components are weaker than the main Doppler component. Figure 21 shows the STFT spectrogram using a Hanning window of 128 samples. In total, there are two HERM lines which appear as five lines in Fig 21. Note that for $M$ HERM lines the total number of lines in the spectrum is $2 M+1$.

Next, the SNR of the HERM line components were varied from 0 to $20 \mathrm{~dB}$, and the mean and the variance of the estimates of $2 M+1$ were computed from 1000 iterations. This is shown in Figure 22. For all three methods, a data segment of 128 samples was used to generate a 20 by 20 covariance matrix. This means that the maximum detectable number of positive harmonic components is 20. Given that the PRF is 1.5 $\mathrm{kHz}$ and the HERM line separation of flying drones is between $100-300 \mathrm{~Hz}$, this is a valid assumption that follows from Equation 29.

Both MDL and AIC underestimate the number of harmonic components at low SNR making them poor feature estimation techniques for the total number of HERM lines. AIC performs poorly by overestimating the number of harmonic components even at high SNR. However, the MDL estimate tends to 1 at an SNR of $0 \mathrm{~dB}$ meaning that only the main Doppler component is detected. The Radoi method performs similarly to MDL except it overestimates the number of harmonic components at low SNR. 


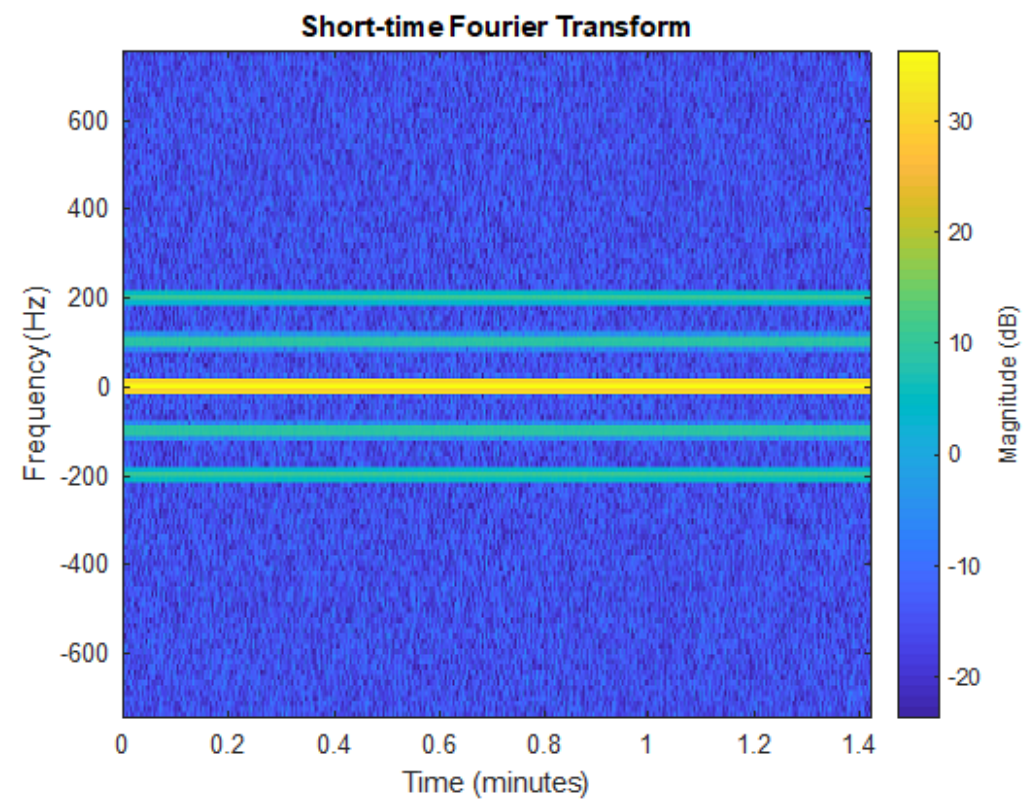

Figure 21: Simulated HERM line signature.

The estimated number of harmonic components can be used as an input to superresolution techniques such as Multiple Signal Classification (MUSIC). This is because MUSIC requires knowledge about the number of frequency components present in the signal. The frequency estimates obtained from MUSIC can be further refined by rejecting the outliers. Thus, overestimation of the number of harmonic components is not a major shortcoming. Thus, the Radoi method is the preferred method for estimating the number of HERM components.

\subsubsection{Multi-Rotor Drone}

In the case of a multi-rotor drone, the propellers may spin at different rates leading to more than one set of HERM lines. Multiple spinning rates simply add additional sinusoidal components to Equation 33. Thus, these signal subspace dimension estimators will just estimate the total number of harmonic components $(M \times C)$. 

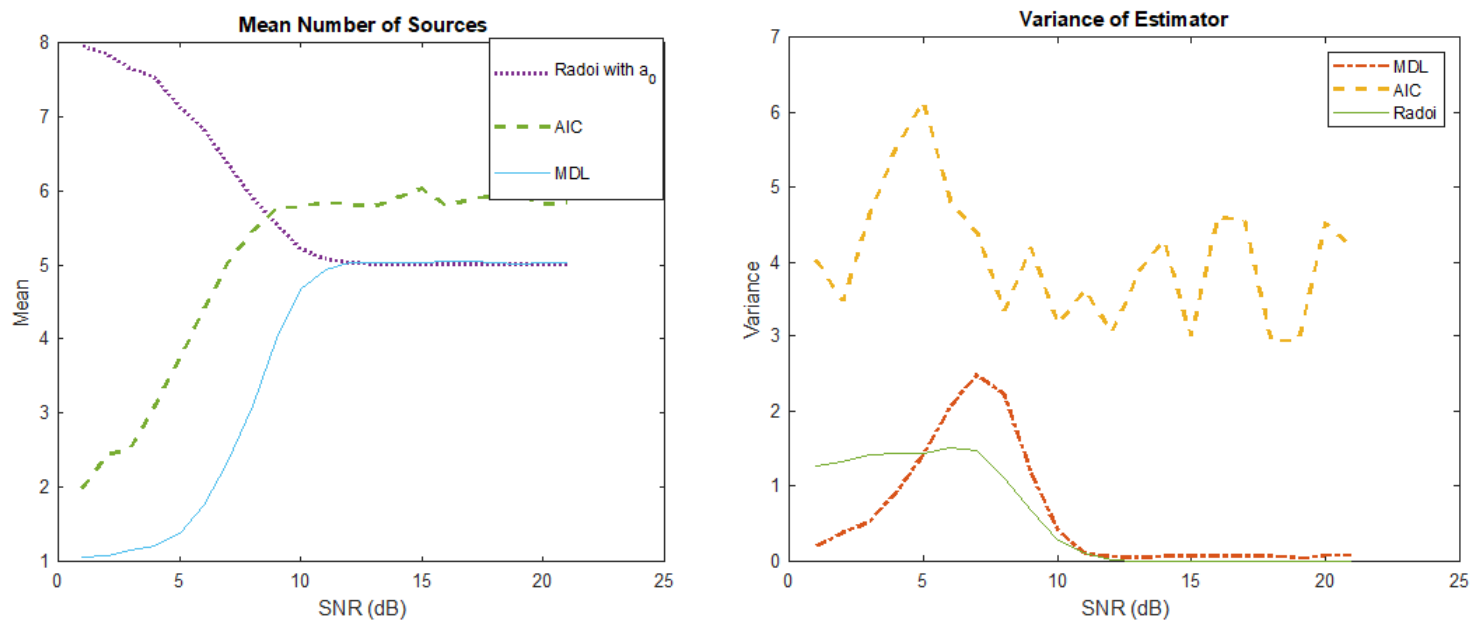

Figure 22: Mean and variance of the estimate of the number of harmonic components using MDL, AIC and Radoi.

The time series model in Equation 33 with two fundamental frequencies at $100 \mathrm{~Hz}$ and $150 \mathrm{~Hz}$ was used to model the micro-Doppler signal received from a multi-rotor drone. The parameters are shown in the Table 8.

In total, there are four HERM lines and two fundamental frequencies leading to nine harmonic components. Figure 23 shows the mean estimate of the number of harmonic components using the MDL criteria with varying covariance matrix size $L \in[20,30,40]$ and using a data segment length of $N=128$ samples. The trajectory matrix is of size $L \times(N-L+1)$ of the form

$$
\mathbf{Y}=\left[\begin{array}{cccc}
x[1] & x[2] & \cdots & x[N-L+1] \\
x[2] & x[3] & \cdots & x[N-L+2] \\
\vdots & \vdots & \ddots & \vdots \\
x[L] & x[L+1] & \cdots & x[N]
\end{array}\right]
$$

so that the covariance matrix is $\frac{1}{(N-L+1)} \mathbf{Y} \mathbf{Y}^{H}$ is an $L \times L$ matrix. Note that $L$ is the data window size and $N$ is data length (snapshot) for which stationarity is assumed. $H$ stands for hermitian transposition operation. All three covariance matrices were 
Table 8: Multi-rotor HERM line model parameters.

\begin{tabular}{|l|l|}
\hline Parameter & Value \\
\hline$a_{0}$ & 1 \\
\hline$a_{n}$ & 0.1 \\
\hline$f_{01}$ & 100 \\
\hline$f_{02}$ & 150 \\
\hline$M$ & 2 \\
\hline$C$ & 2 \\
\hline $\mathrm{PRF}$ & $1.5 \mathrm{KHz}$ \\
\hline
\end{tabular}

able to obtain a mean estimate of harmonic components of 9 at high SNR. However, using a $20 \times 20$ covariance matrix failed to correctly estimate the number of harmonic components at an SNR of $10 \mathrm{~dB}$. This demonstrates that for closely spaced frequencies, a larger covariance matrix is needed to provide good estimates of the number of harmonic components. The minimum covariance matrix size to resolve two frequencies $\Delta f$ apart is

$$
L=\frac{B}{\Delta f}
$$

where $B$ is the Doppler bandwidth and is equal to the PRF of the radar. $\Delta f$ is taken as the minimum HERM line separation frequency. Using a priori information, this can be as $50 \mathrm{~Hz}$ in this example along with the PRF as $1.5 \mathrm{KHz}$.

\subsection{Experimental Results}

\subsubsection{Single Rotor Drone}

In this section, the performance of using the Radoi method to estimate the number of harmonic components is compared to a HERM line spectrogram peak detection 

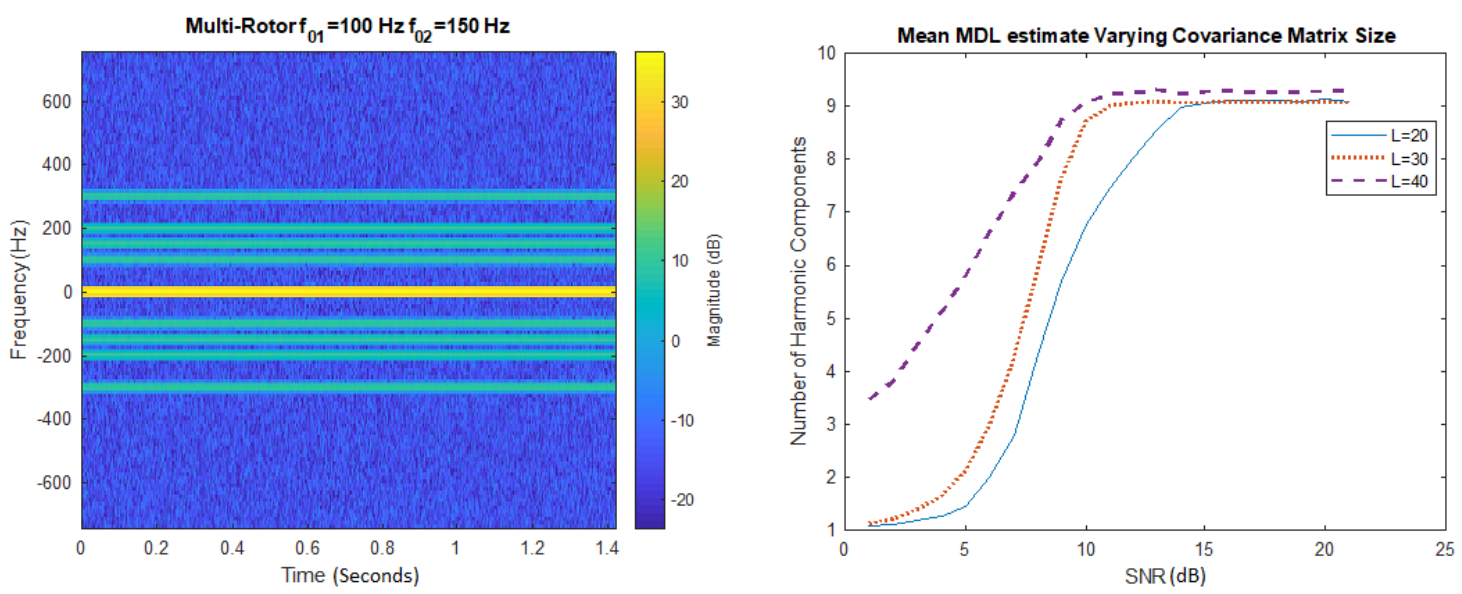

Figure 23: Mean estimate using MDL with varying covariance matrix sizes for a case of two fundamental frequencies.

method [39] for a single rotor drone. The spectrogram shown in Figure 24 was obtained using the Xethru radar where the drone was placed $75 \mathrm{~cm}$ away from it. Since the drone is very small (also its RCS) and the transmitted power is low, weak microDoppler signatures were obtained at this range.

The MATLAB findpeaks method is used to estimate the number of HERM lines. First, a Hanning window is applied to a data segment and a FFT is applied on the windowed data to generate the HERM line spectrum. Then the findpeaks function is applied to find the number of peaks. The minimum peak width is selected to be $25 \mathrm{~Hz}$ and the minimum peak amplitude is set to be $-50 \mathrm{~dB}$ (noise floor). Figure 24 shows the estimated number of harmonic components using the Radoi method and the spectrogram peak detection method. For both methods, a data segment of length $128(\approx 0.085 \mathrm{~s})$ is used. The mean and variance of the estimated number of HERM lines is computed for both methods and shown in Table 9. Since the rotor is only spinning at steady state between 1 and 5 seconds, only the estimates within this time frame are considered. The method by Radoi has lower variance than the spectrogram peak detection method. For feature extraction purposes, it is important to have features with low variance. 
Table 9: Estimated number of HERM lines using Radoi and Spectrogram Peak Detection Method.

\begin{tabular}{|l|l|l|}
\hline Method & Mean & Variance \\
\hline Radoi & 9.78 & 1.15 \\
\hline Peak Detection & 8.7 & 2.92 \\
\hline
\end{tabular}
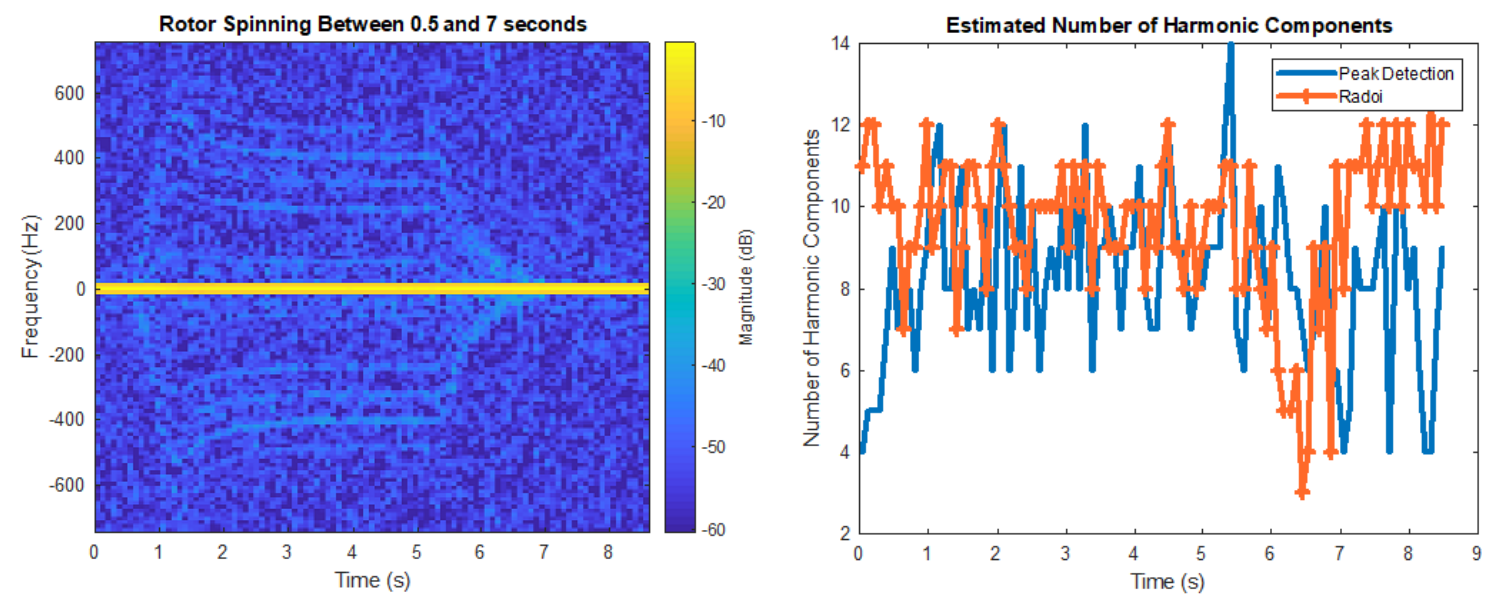

Figure 24: Left: Spectrogram. Right: Estimated number of harmonic components using Radoi method on the time series as compared to the spectrogram peak detection method.

Thus, the Radoi method may be an alternative way of estimating the total number of HERM lines. Note that the Radoi method still estimates that there are harmonic components after 7 seconds even when there are no HERM lines. This is case in Figure 22 as well, i.e., the mean Radoi estimate overestimates the number of HERM lines to be 8 harmonic components at a SNR of $0 \mathrm{~dB}$ when it should be 0 . This suggests that the Radoi method should only be used for feature extraction purposes once it is certain the target is a drone. 


\subsubsection{Multi-rotor drone}

Figure 25 shows an example of the estimated number of harmonic components using these parametric spectral estimation techniques for the Typhoon $\mathrm{H}$ hexacopter fixed on a stand $2 \mathrm{~m}$ away with rotors spinning at different rates captured using the Xethru $\mathrm{X} 4$ radar. Before 5 seconds, all the rotors were spinning at the same rate so there were only 4 harmonic components. Afterwards, the rotors started to spinning at different rates $(C>1)$ which introduced more harmonic components. As shown in Figure 25, the estimated number of harmonic components did increase accordingly after 5 seconds. Both the MDL criterion and the Radoi method were applied using a
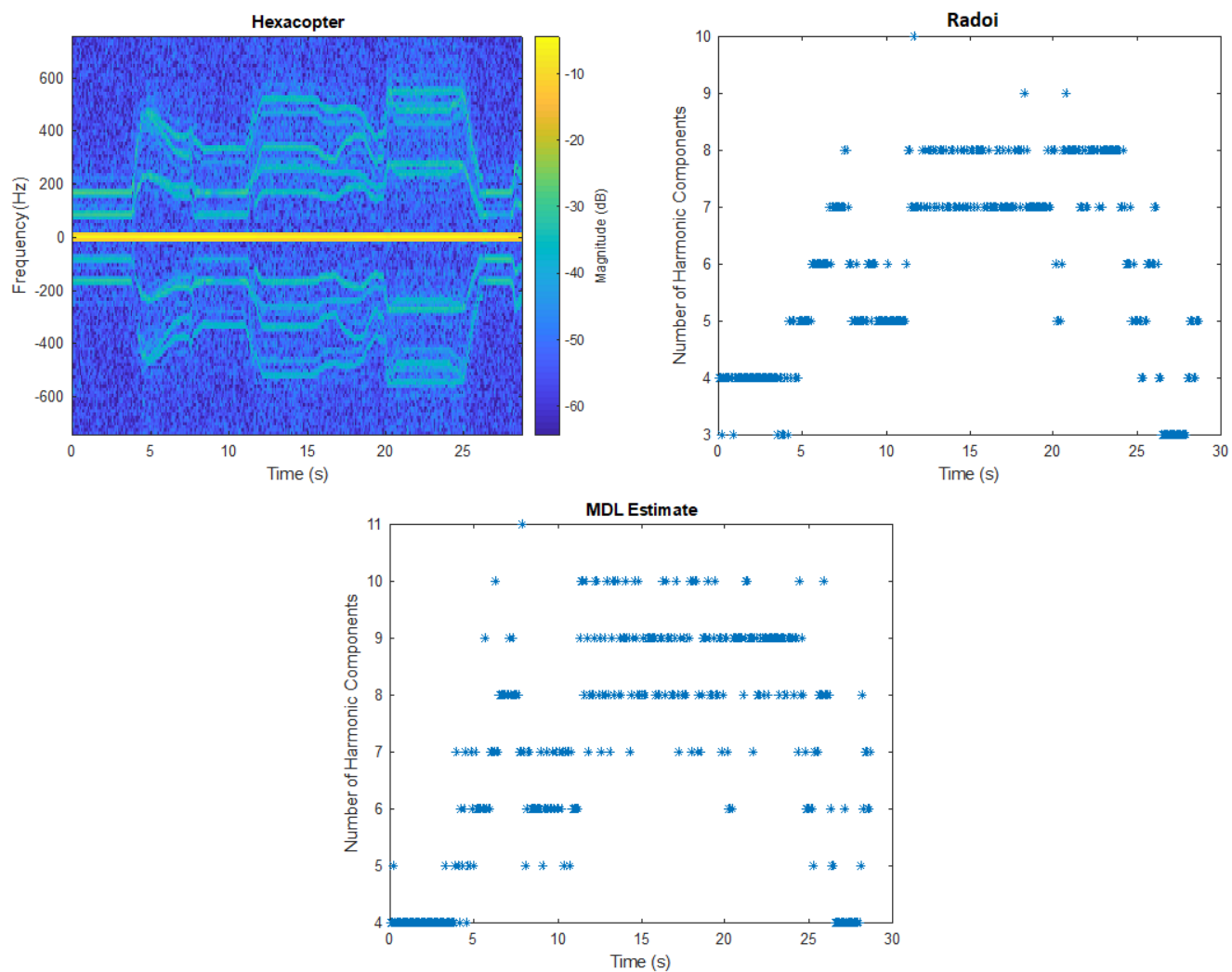

Figure 25: Estimated number of harmonic components using Radoi and MDL for Hexacopter with multiple fundamental frequencies. 
30 by 30 covariance matrix generated using a window of 128 samples. Since the SNR is high, both methods should perform similarly (see Figure 22). However, the MDL criterion actually performs worse with a greater variance. This further demonstrates that the Radoi method is more suitable for estimating the total number of HERM lines than MDL.

\subsection{Applications}

\subsubsection{Rotary Drone Detector}

A rotary drone detector could be proposed using the following criteria

$$
D= \begin{cases}\text { Rotary Drone } & \text { if } n>1 \\ \text { No Rotary Drone } & \text { if } n=1\end{cases}
$$

where $n$ is the number of harmonic components estimated by MDL. If $n=1$, then this implies that only the main Doppler component (i.e., only the body line) is detected and therefore, the target is not a rotary drone.

Recall that the Radoi method is superior to the MDL method for estimating the number of HERM lines. Specifically, the MDL method underestimates the number of HERM lines, while the Radoi method overestimates it. In this thesis, the MDL is approach used for estimating $n$.

The performance of MDL-based detection method can be improved by removing the main Doppler component. Since the main Doppler component is typically 20 to $40 \mathrm{~dB}$ higher than micro-Doppler, this could severely affect the detection of weak HERM line components. This is illustrated in Figure 26 showing the distribution of the ordered eigenvalues. By removing the main Doppler term $a_{0}$, it is visually apparent 

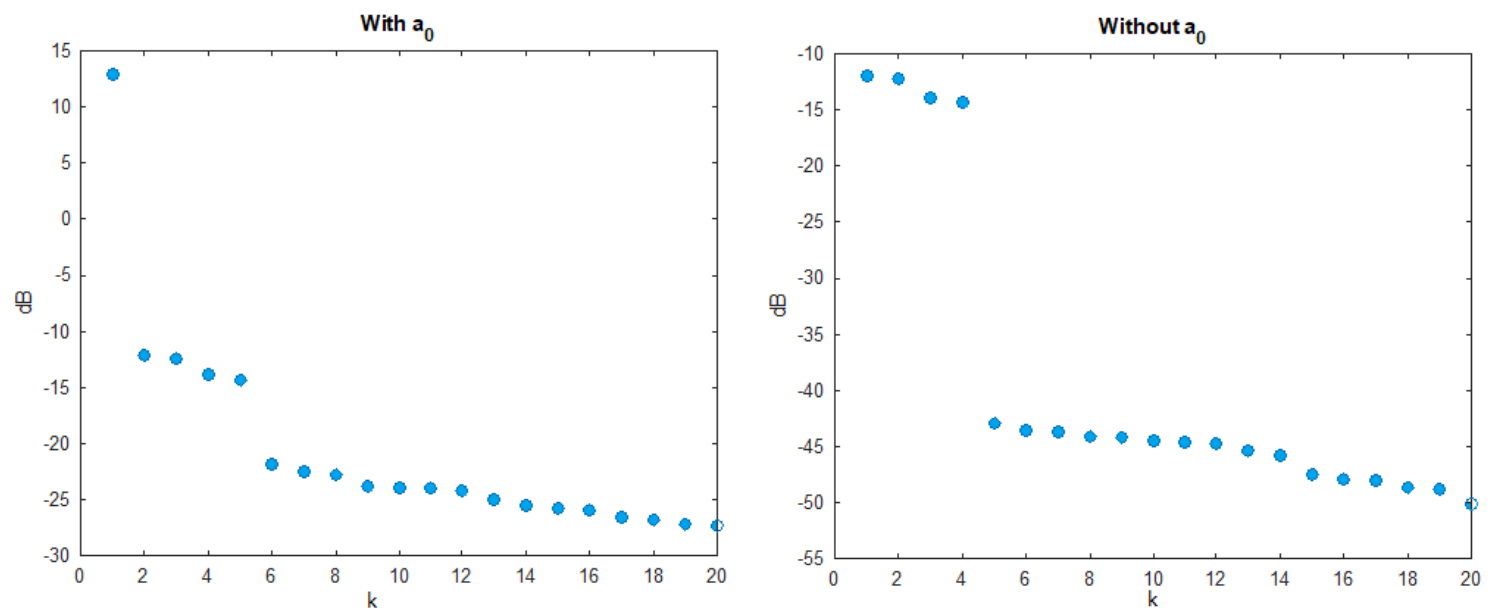

Figure 26: Distribution of eigenvalues with the main Doppler component $a_{0}$ and without.

that the number of harmonic components is 4 . The criteria for this new rotary drone detector becomes:

$$
D= \begin{cases}\text { Rotary Drone } & \text { if } n>0, \\ \text { No Rotary Drone } & \text { if } n=0,\end{cases}
$$

since there should be no harmonic components detected if there are no HERM lines.

Two different techniques were used to remove the main Doppler component. The first method is based on Singular Spectrum Analysis (SSA) and reconstructs the time series ignoring the first principal component which corresponds to the main Doppler component (see Figure 26). This technique was first demonstrated by Clemente to remove the main body component for micro-Doppler signatures of helicopters [40]. First, the principal components are generated by projecting out the components along the dominant eigenvectors in the trajectory matrix $Y$

$$
P C=P_{E} Y, \quad P_{E}=I-E\left(E^{H} E\right)^{-1} E^{H},
$$

where $E$ is a matrix of dominant eigenvectors and $H$ is the hermitian transposition operation. The body line is removed by choosing $E$ to be $E_{1}$ to be the column vector corresponding to the dominant eigenvalue. 
The second technique of removing the main body component involves using an infinite impulse response (IIR) high pass filter with passband at $30 \mathrm{~Hz}$ because the propeller rates are higher than $30 \mathrm{~Hz}$. The same parameters from Table 7 were used to generate the signal and 1000 iterations were run for each SNR level. Figure 27 shows the probability of detection as a function of SNR with and without the removal of the main Doppler component. Both the high pass filter and the SSA method performed equally well and were able to improve the probability of detection at an SNR of $7 \mathrm{~dB}$ from $70 \%$ to $90 \%$. This is plausible because when the body line was included, the eigenvalue corresponding to the body line obscured the contributions of HERM lines while the removal of the body line enabled the HERM line contributions to stand out more clearly. To summarize, the steps of the proposed MDL-based rotary drone

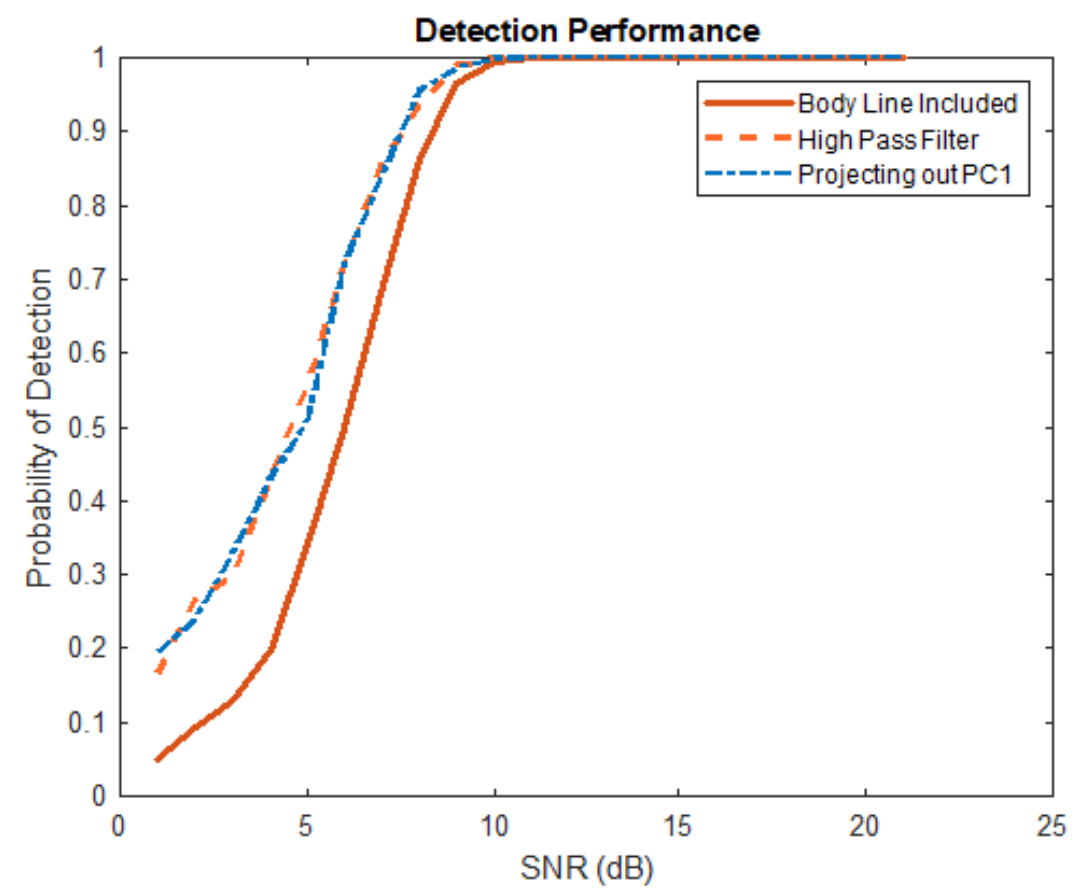

Figure 27: Performance of proposed rotary wing detector with and without removal of the main Doppler component.

detector are: 
1. Identify range bins where there may be a drone using a CFAR (Constant False Alarm Rate) detector.

2. Shift the main body Doppler line to zero Doppler.

3. Apply a high-pass filter (e.g., Butterworth filter order 6) to a segment of $N$ data points in slow time to remove the main Doppler component.

4. Generate a $L \times L$ covariance matrix by first building trajectory matrix $Y$ as shown in Equation 55 and then applying $\frac{1}{(N-L+1)} Y Y^{H}$.

5. Find the eigenvalues of the covariance matrix and use the MDL criterion to estimate the number of harmonic components

6. If this number is more than 0, then HERM lines are present and it is a rotary drone.

The performance of this MDL-based method of detecting micro-Doppler was tested using real data obtained using the Xethru X4 radar and the micro-helicopter drone. An experiment was conducted by illuminating with a Xethru radar a fixed microhelicopter drone at a range of $1 \mathrm{~m}$ for two scenarios, viz., with and without its propeller spinning. The data corresponding to a stationary drone without its propellers spinning is used to represent false alarm where the target has similar RCS but does not exhibit micro-Doppler. Figure 28 shows the STFT spectrogram for each scenario. The proposed MDL-based detector should detect multiple harmonic components within the time period of $0.5 \mathrm{~s}$ and $7 \mathrm{~s}$. The size of the data segment $N$, and the size of the covariance matrix $L$ are two parameters that impact the detection performance. Figure 29 shows the estimated number of harmonic components as the data segment size was varied while $L$ was fixed at 30 .

The best performance was obtained using a data segment size of $N=128$ with a probability of detection of 0.85 and a probability of false alarm of 0.06 . For larger $L$, the assumption of stationarity is no longer valid and this introduces many false 

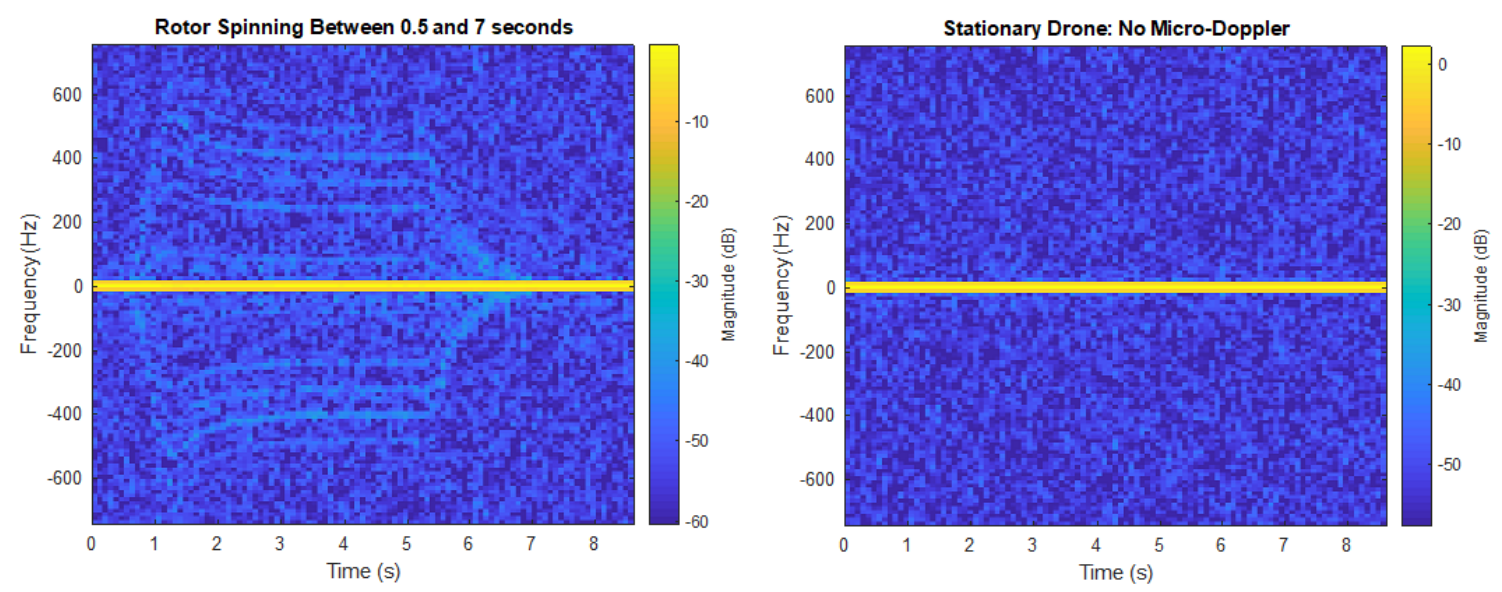

Figure 28: STFT Spectrogram. Left: HERM line spectrogram of the micro-helicopter drone with its rotor spinning, starting from $0.5 \mathrm{~s}$ and stopping at $7 \mathrm{~s}$. Right: Stationary micro-helicopter drone with its rotors at rest.

alarms. As shown in Figure 28, the propeller rotation rate of the micro-helicopter drone is not constant.

Table 10: Detection Performance for MDL for different segment lengths.

\begin{tabular}{|l|l|l|l|}
\hline & $N=64$ & $N=128$ & $N=256$ \\
\hline$P_{d} / P_{f a}$ & $0.4 / 0.16$ & $0.85 / 0.06$ & $0.97 / 0.33$ \\
\hline
\end{tabular}

The window size $L$ (so covariance matrix is of size $L \times L$ ) determines the minimum resolvable spacing between frequency components. Figure 30 shows the MDL estimate with varying $L$ and keeping the data segment length $N$ is fixed at 128 samples.

As $L$ increases, the minimum resolvable frequency spacing between harmonic components decreases. Using $L=20$ underestimated the number of harmonic components and using $L=40$ overestimated the number of harmonic components. Note that $N$ is fixed here (due to stationarity constraint in practice), so that when $L$ is larger, then there are fewer samples to estimate the covariance matrix. The best performance was 


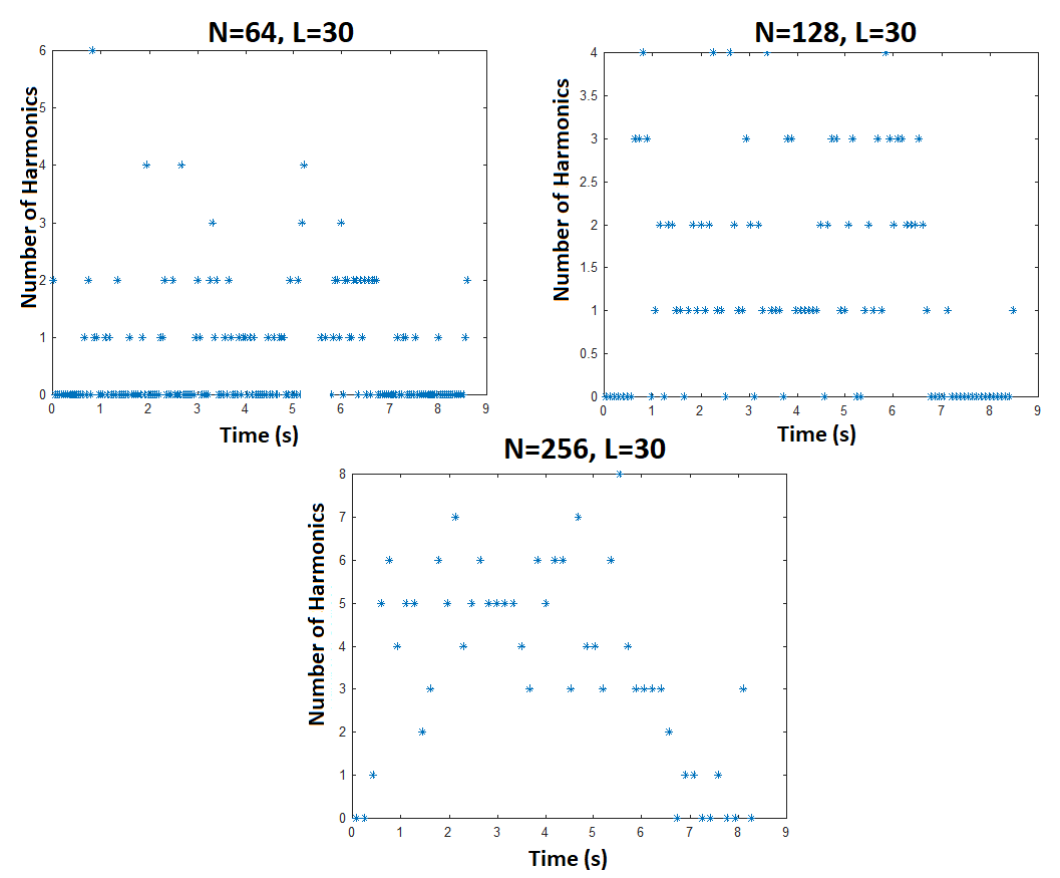

Figure 29: Estimated number of harmonic components using MDL detector with varying window size and a $30 \times 30$ covariance matrix.

obtained using a $L=30$ and $N=128$ resulting in a $P_{d}$ of 0.85 and a $P_{f a}$ of 0.06 .

Table 11: Detection Performance using MDL for different covariance matrix size $L$.

\begin{tabular}{|l|l|l|l|}
\hline & $L=20$ & $L=30$ & $L=40$ \\
\hline$P_{d} / P_{f a}$ & $0.4 / 0$ & $0.85 / 0.06$ & $1 / 1$ \\
\hline
\end{tabular}

The performance of this detection method was compared to a STFT power spectrogrambased method. Ideally, the power of the strongest HERM line component in the spectrogram should exceed the threshold corresponding to false alarm from a drone with non-rotating blades. Both MDL-based method and spectrogram-based method used non-overlapping data window size of $N=128$ samples. Figure 31 shows a comparison of the power distributions of the strongest HERM line component with 

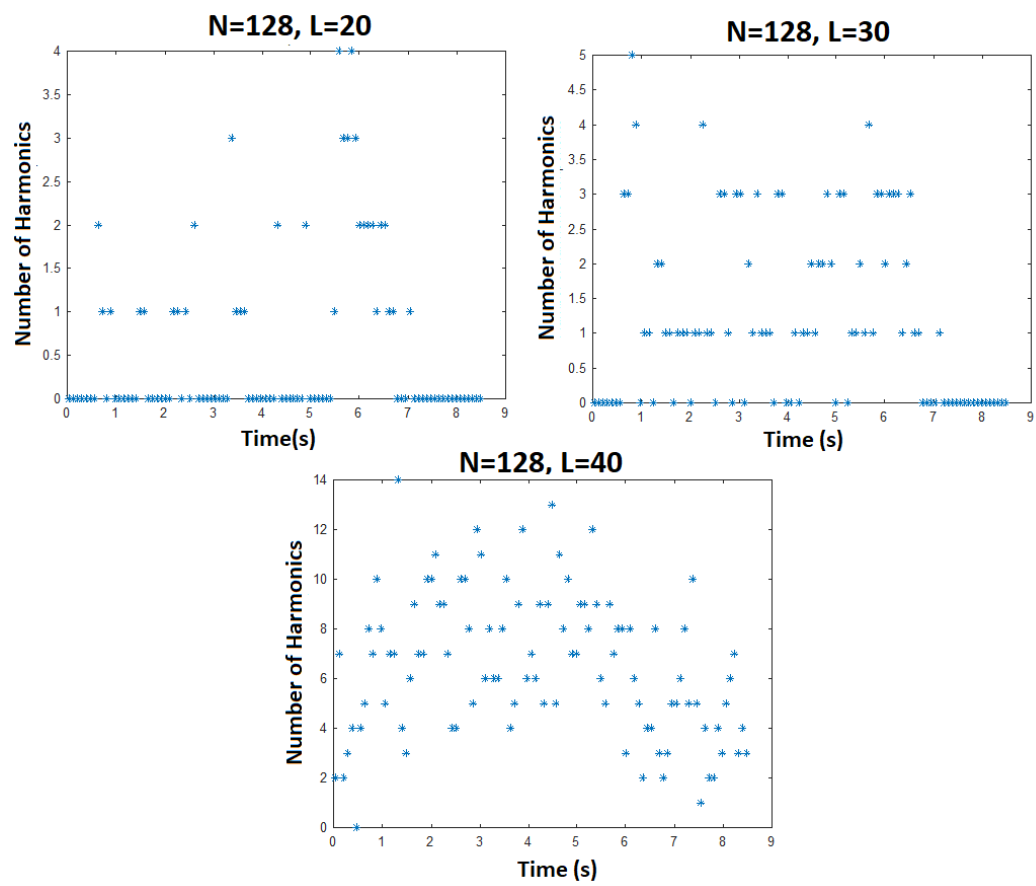

Figure 30: Estimated number of harmonic components using MDL with varying the size of the covariance matrix.

data corresponding to drone with non-rotating blades during the time period of 0.5 and 7 seconds. According to the ROC (Receiver Operating Characteristic) curve, the probability of detection was about 0.85 at a probability of false alarm 0.2 which is worse than the MDL-based using $L=30$ and $N=128\left(P_{d}=0.85, P_{f a}=0.06\right)$. The histogram distribution for the MDL based detector is shown in Figure 32. Both histograms in Figure 31 and Figure 32 are normalized to show the PDF (probability density function). There is much better separability using the MDL-based detection method. 

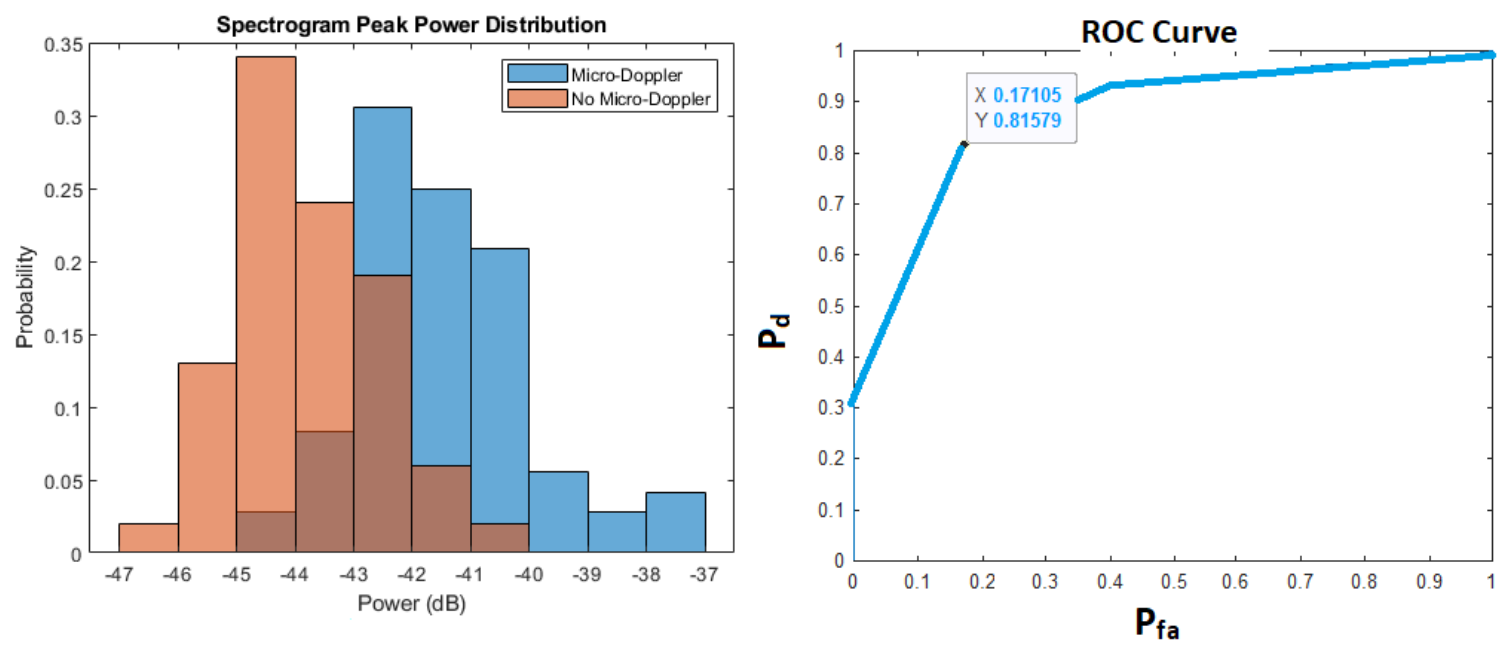

Figure 31: Spectrogram-based detection method. Left: histogram power distribution and Right: ROC curve.

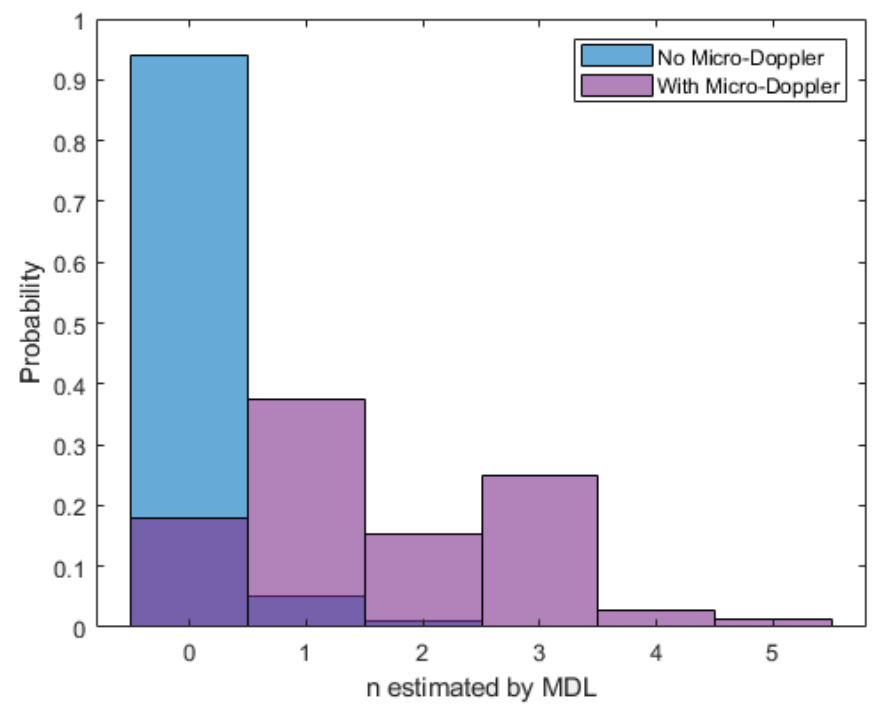

Figure 32: Histogram distribution for MDL-based detector using $\mathrm{M}=30$ and $\mathrm{L}=128$. 
To further illustrate the detection potential of the MDL-based method, Figure 33 shows the full 2-D radar matrix of the detections obtained using smallest-of cell averaging (SOCA) CFAR detector and the detections obtained using the proposed method for the above experiment. A SOCA CFAR detector was used since the target was close to the receiver. For the CFAR detector, 5 training cells were used with 3 guard cells and a $P_{f a}$ of 0.07 . There are many false alarms with just using CFAR to detect the drone. The proposed MDL-based detection method was then applied on all the range bins detected by CFAR to look for the presence of micro-Doppler. The proposed method was able to correctly identify the drone at range bins 9 to 11 with a $P_{d}$ of 0.85 and a $P_{f a}$ of 0.06 . This false alarm rate remained constant for all the range bins.

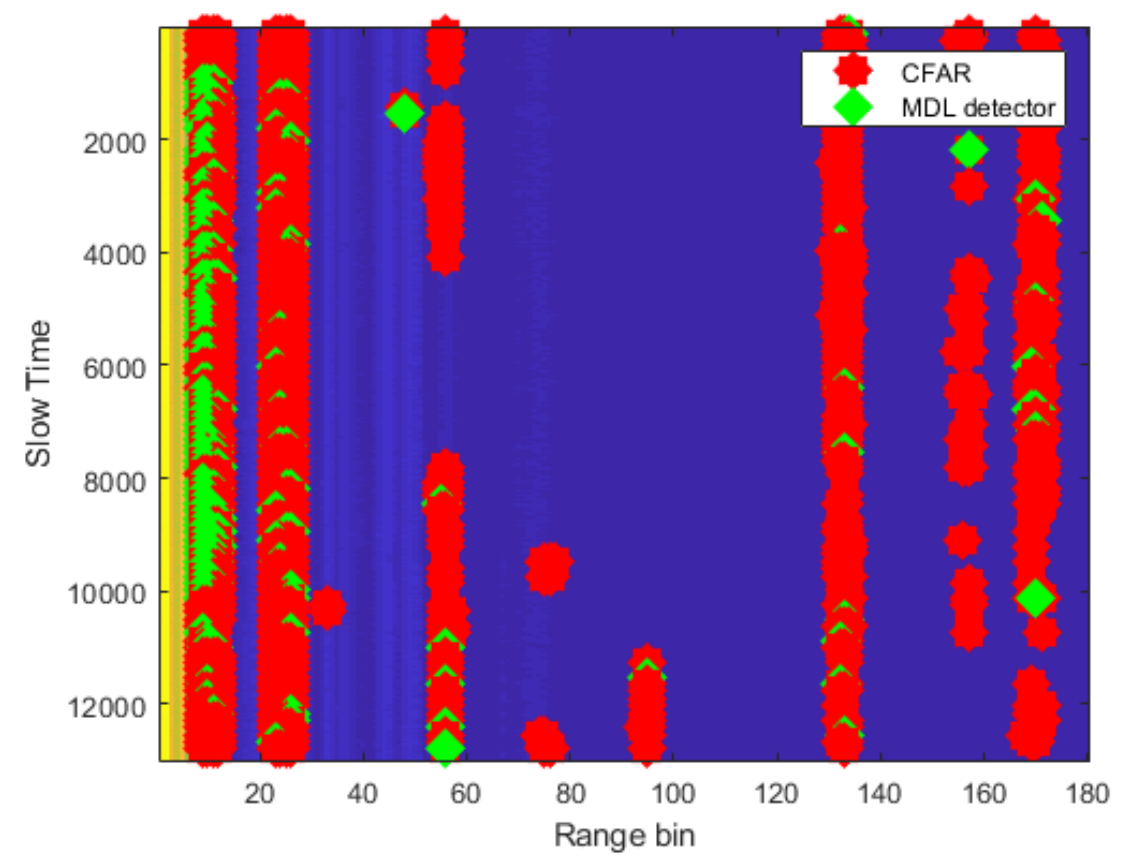

Figure 33: 2-D radar matrix showing the CFAR detections and the detections using the MDL detector. 


\subsection{Computational Complexity}

In this section, the complexity of the covariance matrix-based methods are examined. All of these methods require the computation of a covariance matrix and eigen decomposition which has a complexity of $\mathrm{O}\left(L^{2}(N-L+1)+L^{3}\right)$ where $N$ is the data segment size and L is the size of the covariance matrix. It's important to follow Equation 54 when determining the size of the covariance matrix. Generally, a higher PRF means that a larger covariance matrix is required to obtain the same frequency resolution. In comparison, the complexity of a simple spectrogram peak detection method requires computing a $N$ point FFT which only has a complexity of $\mathrm{O}(N \log (N))$.

\subsection{Conclusion}

In this chapter, covariance matrix-based methods for estimating the number of HERM lines were explored. A parametric method of estimating the number of HERM line components based on the eigendecomposition of the covariance matrix was proposed. It was found that popular techniques, like MDL and AIC, underestimated the number of HERM line components at low SNR since the HERM line components are much weaker than the main Doppler shift. A rotary drone detection method, by first filtering out the main Doppler component (or projecting it out) and then applying MDL, was proposed. If the MDL estimate is greater than 0 then the proposed method declares that the target is present since it implies HERM lines are present.

Moreover, the novel technique by Radoi [41] had the interesting characteristic of overestimating the number of the harmonic components at low SNR. Following the detection process, the Radoi method was used to estimate the number of harmonic components. This could potentially be used as an alternate method to spectrogrambased peak detection methods for estimating the total number of HERM lines. The 
Radoi method does not require any prior knowledge about the signal and is shown to provide estimates with lower variance than Fourier-based peak detection methods. 


\section{Chapter 5}

\section{Estimating HERM line Fundamental Frequency}

Besides the number of HERM lines, important information can be derived from the frequencies of the harmonic components in the HERM line signature. For a single rotor drone, an important feature is the HERM line separation frequency or the fundamental frequency. The fundamental frequency is an estimate of the microDoppler periodicity and can be used to judge how fast the drone is moving.

In this chapter, two methods are proposed to estimate the fundamental frequencies in the HERM line spectrum. The performance of these method are compared to other Fourier-based methods of estimating the propeller rotation rate, namely the cepstrum and the cadence-velocity diagram. In the end, drone classification using 2 features-the number of HERM lines and the fundamental frequency is shown using a Naive Bayes classifier. 


\subsection{Existing Methods}

\subsubsection{Cepstrum}

The cepstrum is a method that has been proposed to estimate the micro-Doppler periodicity when the PRF of the radar is not sufficiently high [10]. The cepstrum was first introduced by Bogart et al. in 1963 [42] and its use has been popularized in speech processing and seismology. The power cepstrum is defined as the inverse Fourier transform of the log of the power spectrum:

$$
C(q f t)=\left|\mathcal{F}^{-1} \log \left(|\mathcal{F}(s(t))|^{2}\right)\right|^{2} .
$$

Taking the log operation on the squared magnitude of the Fourier transform compresses the amplitudes of the harmonic peaks and the resulting spectrum can be treated as an amplitude modulated periodic waveform. Taking the inverse Fourier transform will result in a peak at the period of this waveform (quefrency). This is called quefrency since it is a measure of frequency but in the time domain. The fundamental frequency can be derived by $1 / q f t$.

Noll developed the concept of a short-time cepstrogram by applying the short-time Fourier transform and then computing the cepstrum for each segment [23].

$$
C(m, k)=\left|I F F T^{-1} \log \left(|S T F T(s[n])(m, k)|^{2}\right)\right|^{2}
$$

where $m$ is the position of the window and $k$ is the frequency bin of interest. The quefrency with the highest peak in the cepstrogram was used to estimate the rotor periodicity. When the cepstrogram gets corrupted by noise, the highest peak in the cepstrogram may not always represent the rotor periodicity. This is shown in Figure 34. Figure 34 shows an example of a cepstrogram and a cepstrum for a sum of 5 sinusoids with fundamental frequency $80 \mathrm{~Hz}$ corrupted with zero mean white Gaussian noise with $7 \mathrm{~dB}$ variance. The peak at $0.012 \mathrm{~s}$ corresponds to a frequency of $83 \mathrm{~Hz}$ which is consistent with the true fundamental frequency. However, that the 
highest peak in the cepstrum is actually at a quefrency of $0.037 \mathrm{~s}$ which corresponds to a frequency of $27 \mathrm{~Hz}$. This is too low to be a rotation rate of a spinning propeller and can be rejected. However, this demonstrates how noise can corrupt the cepstrum and affect the fundamental frequency estimate. Therefore resorting to picking frequency of the maximum from the cepstrogram may not be the best way of estimating the periodicity of the rotor.
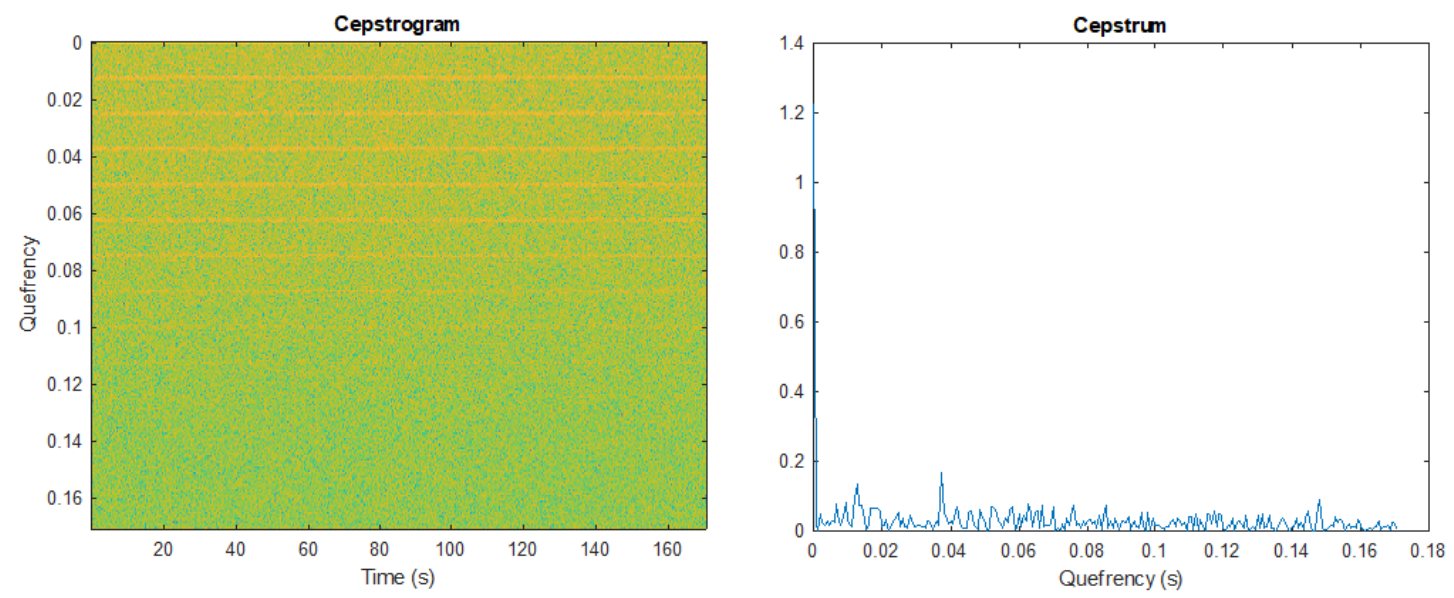

Figure 34: Cepstrogram and cepstrum.

\subsubsection{Cadence-Velocity Diagram}

The cadence velocity diagram (CVD) is another method of studying the periodicity in the STFT micro-Doppler spectrogram by taking the Fourier transform along each Doppler frequency bin in time. The resulting cadence-velocity diagram contains periodic vertical lines and is a function of cadence where the sample rate is $\frac{f_{s}}{\text { (window length) } \times(\text { overlap) }}$. Cadence is a measure of how often a pattern repeats and is in units of Hz. Cadence-velocity spectrum (CVS) is obtained by averaging over the Doppler frequency dimension of the cadence-velocity diagram at each cadence [22]. Figure 35 shows an example of the cadence velocity diagram and the cadence velocity spectrum of a single rotor drone captured using the TI radar. 

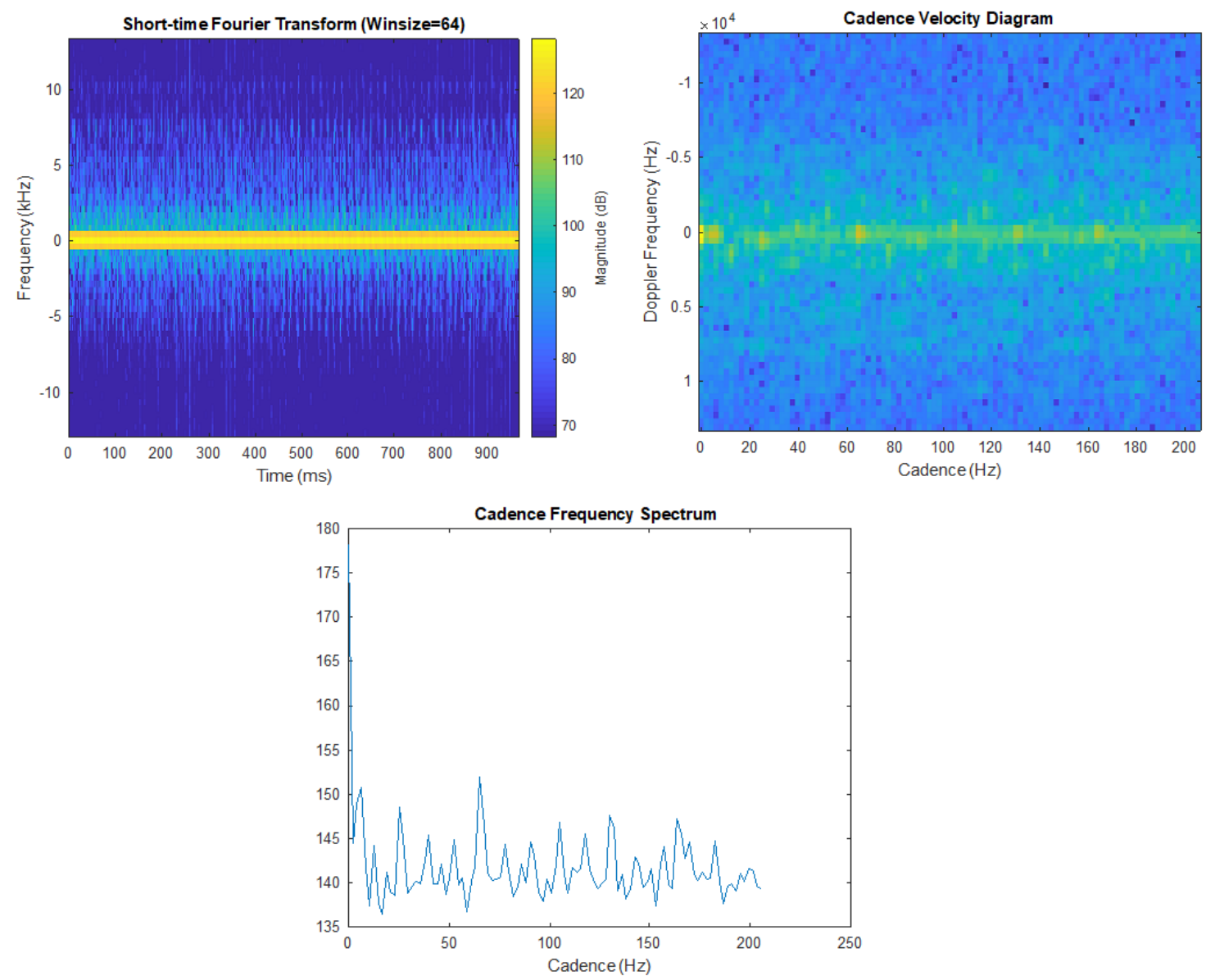

Figure 35: Cadence-velocity diagram and cadence-velocity spectrum of micro-Doppler captured by the TI radar.

Molchanov [43] developed an iterative cadence-velocity diagram-based method of estimating the micro-Doppler periodicity by varying the STFT window size that was used to generate the CVS. If the processed signal has period $T$ then the spectrogram will also have period $T$. Choosing the window size of the STFT to match the micro-Doppler period will optimize the CVS estimate of the period and prevent overestimation. Molchanov used an initial STFT window size of $L=2$ to generate the CVS and varied $L$ by a power of 2 . All the possible cadence velocity spectrums such that the window length $L$ is less than the data segment length $I$ is generated. The 
cadence velocity spectrum which had the highest peak is used for the final fundamental frequency estimate. The cadence corresponding to the highest peak is the fundamental frequency estimate.

The steps of Molchanov's method are listed below.

1. Generate the STFT power spectrogram of the micro-Doppler signal using window size of $L$.

2. Apply a Fourier transform across the time dimension of the spectrogram to generate the CVD. Then average over the Doppler frequency dimension of the CVD at each cadence to generate the CVS for the window size $L$.

3. Check if the next window size $L=2^{1+\log _{2} L}$ is less than the data segment length I. If so, repeat steps 1 and 2 to generate the CVS for the new window size. Keep a collection of all the possible CVS.

4. Once all the possible CVS are computed, pick the CVS with the highest peak. This corresponds to the most optimal window size. The fundamental frequency estimate is just the frequency of the highest peak in the chosen CVS.

Molchanov demonstrated that this method was able to accurately estimate the propeller rotation rate of a micro-helicopter drone using a high PRF radar of $22 \mathrm{kHz}$ [43]. However, this technique may not perform well for a low PRF radar where clear short-windowed STFT spectrograms cannot be realized. 


\subsection{Proposed Methods}

\subsubsection{Log Harmonic Summation}

The log harmonic summation algorithm is a method borrowed from speech processing. The log harmonic summation algorithm uses a template matching technique to sum up harmonics in the log frequency domain [44]. In the log frequency domain, the harmonics are always at a fixed log spacing away from the fundamental frequency regardless of the frequency. As shown in Figure 36, the first harmonic is $\log (2)$ from the fundamental, the second harmonic is $\log (3)$ from the fundamental and so on.

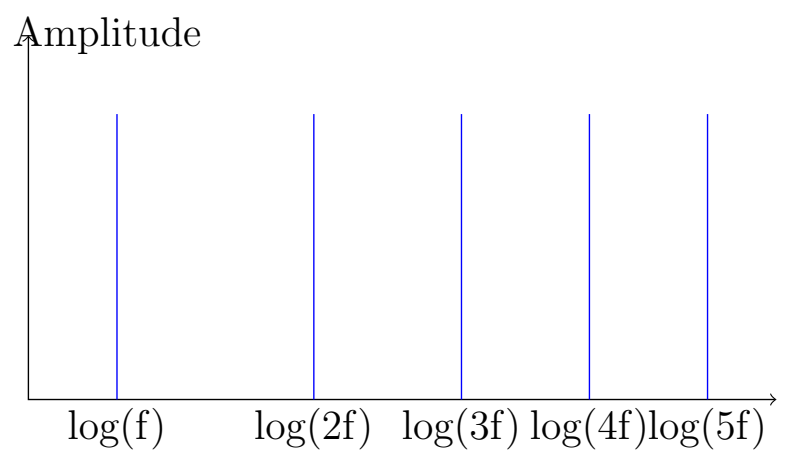

Figure 36: Pattern of harmonics in log frequency scale.

A moving summation of four point fixed log space window is used to add the amplitudes of four harmonics of every frequency in the HERM line spectrum. The fundamental frequency estimate is then based on the frequency which has the strongest harmonics.

Figure 37 shows the steps of the log harmonic summation algorithm. First, a STFT 
of sufficient window size is applied to obtain a HERM line signature. Next, the discrete FFT bins are interpolated to constant-spaced log frequency grid before crosscorrelating with the filter in Figure 36. In order to obtain constant spaced log fre-

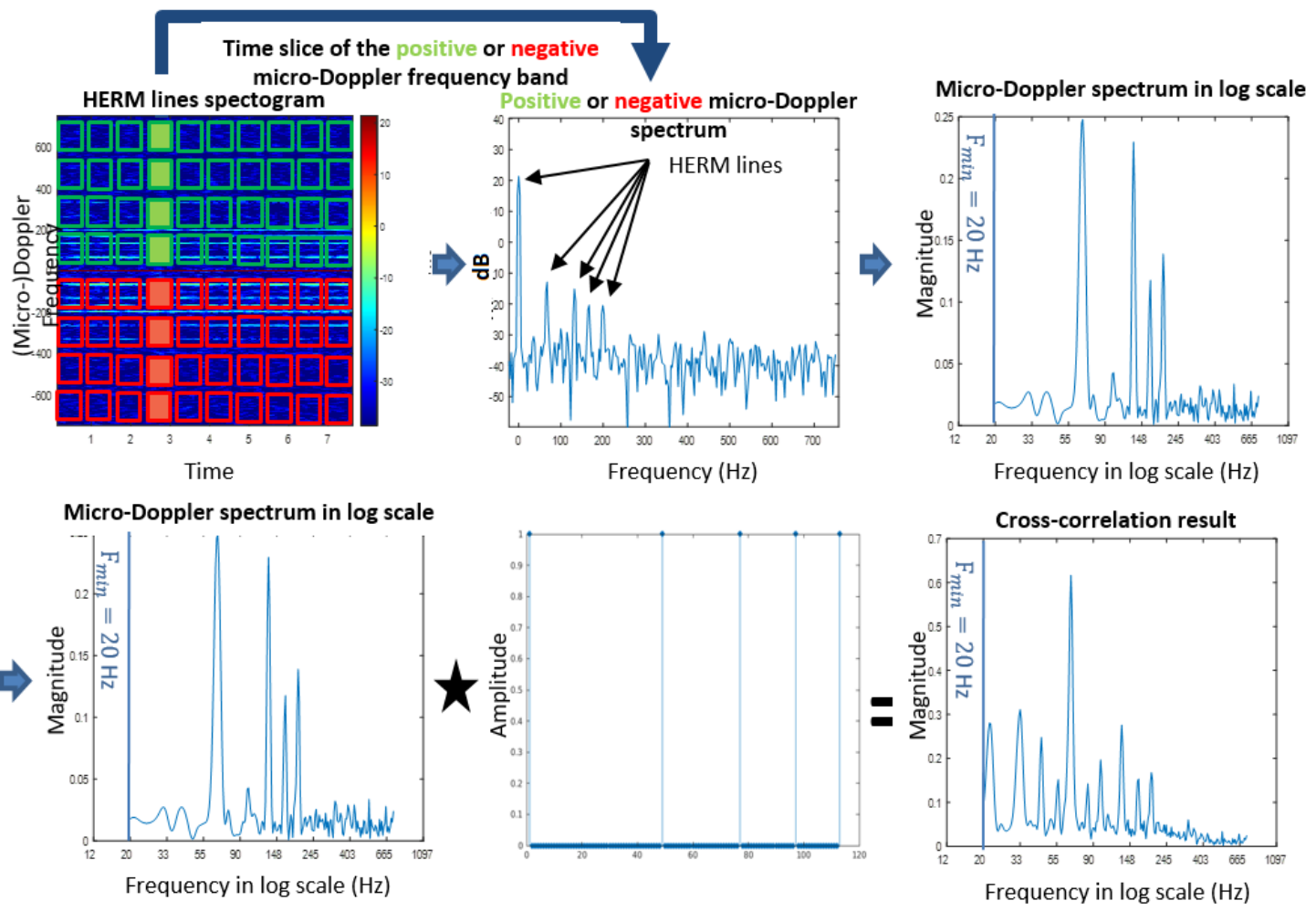

Figure 37: Processing steps of the log harmonic summation algorithm.

quency bins, the frequency bins must be exponentially spaced with the $k$ - th frequency bin being

$$
f_{k}=\left(2^{1 / b}\right)^{k} f_{\min }
$$

where $f_{\min }$ is the minimum frequency and $b$ is the number of frequency bins per octave [45]. The minimum frequency is chosen to be $20 \mathrm{~Hz}$ to ignore micro-Doppler artifacts from the main Doppler component.

The pseudocode for generating this new frequency grid is shown in Algorithm 1 where $f_{\min }$ is the minimum frequency, $f_{\max }$ is the maximum frequency and $B P O$ is 
the number of bins per octave.

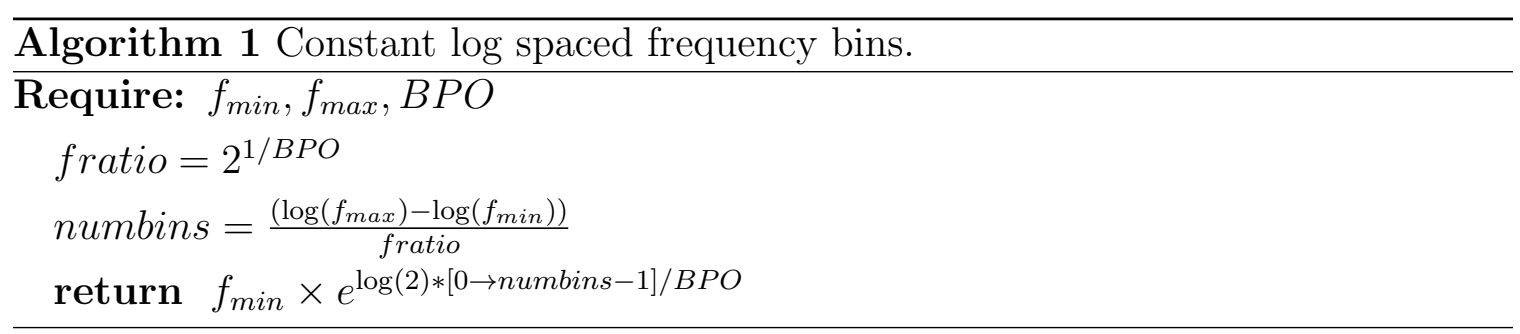

The interpolation of each STFT frame to this new logarithmic grid was done using a cubic spline. The result is then cross-correlated with the delta function filter with spacing shown in Figure 36. The number of zeroes between the delta functions is found by dividing the log spacing between the harmonics by the spacing of the log spaced grid. For example, the number of zeroes between the first and second harmonic is found by:

$$
n=\operatorname{round}\left(\frac{\log (3)-\log (2)}{\log \left(2^{1 / B P O}\right)}\right)
$$

where $B P O$ is the bins per octave.

The frequency with the highest peak in the cross-correlation result is the estimated fundamental frequency. The pseudocode for the log harmonic summation algorithm is shown in Algorithm 2 where

$$
\begin{aligned}
\text { data } & =\text { radar } \mathrm{I} / \mathrm{Q} \text { data in a specific range bin } \\
B P O & =\text { Bins per Octave } \\
F m i n & =\text { Minimum fundamental frequency } \\
P R F & =\text { Sample rate of the signal } \\
\text { numharmonic } & =\text { Number of harmonics to be summed up }
\end{aligned}
$$

For the Xethru $\mathrm{X} 4$ radar, $P R F$ is $1.5 \mathrm{kHz}$ and a window of 512 samples $(0.33 \mathrm{~s})$ is used for each fundamental frequency estimate. 48 bins per octave is used so that there are 222 frequency bins between 30 and $750 \mathrm{~Hz}$. 


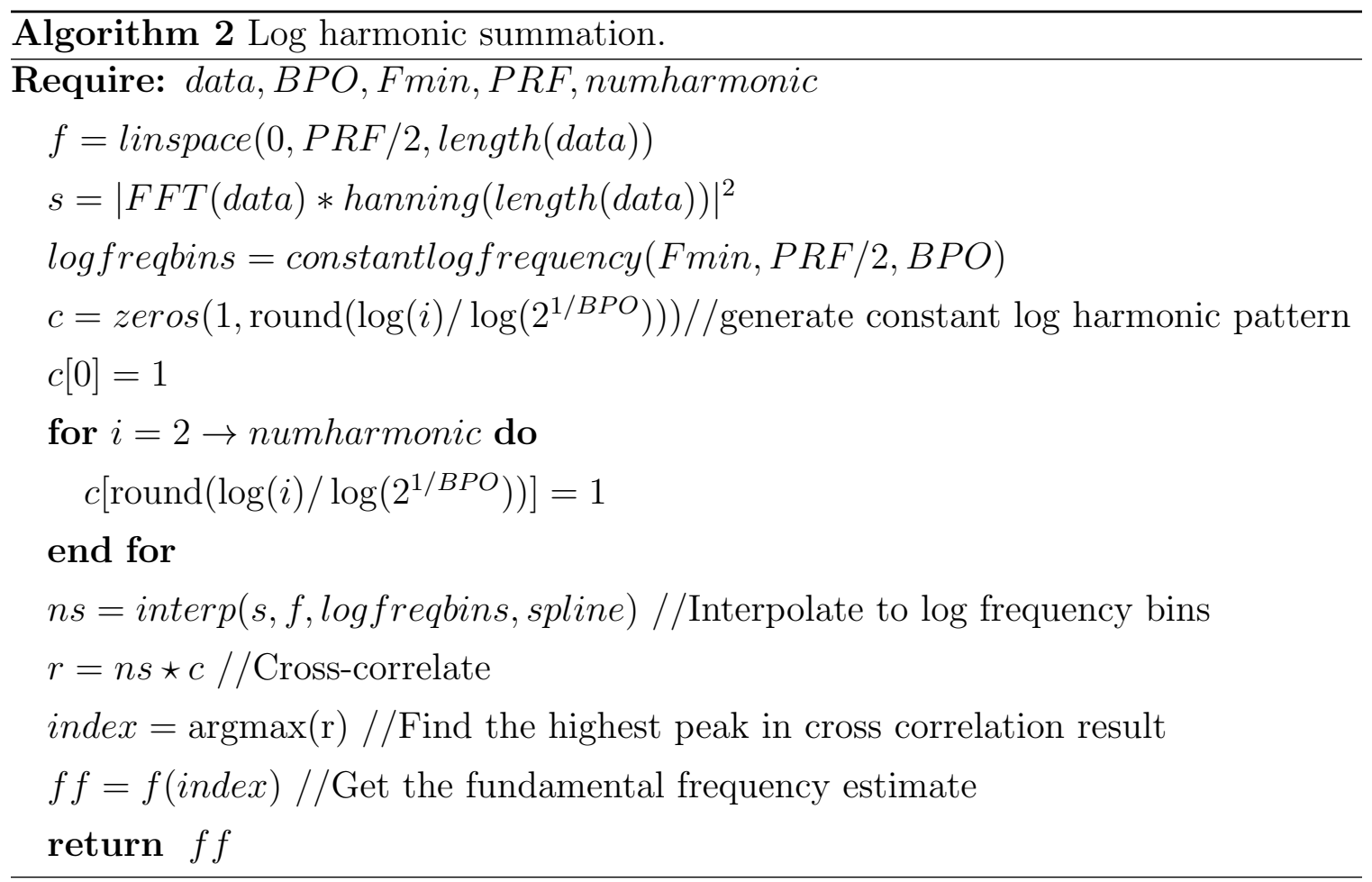

\subsubsection{Fundamental Frequency Estimation using MUSIC}

MUSIC is a superresolution method of estimating the frequencies of a sum of sinusoids based on the eigenvectors of the covariance matrix. MUSIC algorithm become a popular algorithm due its applications in array processing.

Recall that for a sum of $M$ sinusoids in Gaussian noise, the eigenvalues of the sample covariance matrix will contain $M$ values corresponding to the signal space with the rest corresponding to the noise subspace. Since the noise and signal subspaces are orthogonal to each other, then the following relationship is true.

$$
\mathbf{a}^{\mathrm{H}} \mathbf{v}=\mathbf{0}
$$

where $\mathbf{v}$ is a matrix of eigenvectors corresponding to the noise subspace and $\mathbf{a}$ is a steering vector of the signal frequency search space. In the case of estimating the frequencies of the HERM line components, the frequency search space is just all the 
frequencies across the Doppler bandwidth.

MUSIC uses the reciprocal of the squared norm to generate a pseudospectrum of the frequencies in the signal.

$$
P_{M U S I C}(f)=\frac{1}{a^{H} v_{i} v_{i}^{H} a}=\frac{1}{\sum_{i=p+1}^{L}\left|a^{H} v_{i}\right|^{2}}
$$

The frequencies where the squared norm is approximately zero will result in a large peak. The frequencies of the $M$ highest peaks in the pseudospectrum are chosen to represent the frequencies of the $M$ harmonic components in the signal. The number of harmonic components can be estimated using the techniques presented in the previous section. MUSIC is known to provide better frequency estimates than picking peaks in the Fourier transform when the number of harmonic components are known. Kim et al [46] showed that using the short-time MUSIC spectrogram was able to produce clearer drone micro-Doppler signatures than using the STFT or the Wigner-Ville distribution. In this work, root MUSIC was used rather than picking peaks in the MUSIC pseudospectrum to estimate the frequencies of the HERM line components. Root MUSIC uses the roots of the polynomial generated by the squared norm expression in the denominator of Equation 65 to estimate the frequencies of the harmonic components in the signal as shown below:

$$
J(z)=a^{H} v_{i} v_{i}^{H} a=0 .
$$

The $M$ roots closest to the unit circle are chosen as the harmonic components. The frequencies are then estimated using the phase of the complex roots. Root MUSIC is known to perform better than MUSIC for a uniform linear array or a one dimensional grid of frequencies which is the case of the HERM line spectrum [47].

Fundamental frequency estimation using MUSIC is done by first generating a list of fundamental frequency estimate candidates $f_{0} . f_{0}$ is made up of the differences between the ordered frequencies found by MUSIC and the minimum frequency. Next, 
these frequencies are sorted and the fundamental frequency candidates which are a multiple of another are then removed. The remaining fundamental frequency candidates are then evaluated based on how well they fit the vector of frequencies found by root MUSIC. The criteria used is:

$$
C(k)=\sum_{i=1}^{M}\left|\frac{F_{i}}{f_{k}}-\operatorname{round}\left(\frac{F_{i}}{f_{k}}\right)\right|
$$

where $\left\{F_{i}\right\}_{i=1 . . M}$ is the vector of frequencies found by root MUSIC and $f_{k}$ is the fundamental frequency candidate in the set of possible $f_{0}$. The fundamental frequency candidate which minimizes this criteria is selected to be the fundamental frequency.

$$
f_{s}=f_{\min _{k}(C)}
$$

The flowchart of steps is shown in Figure 38.

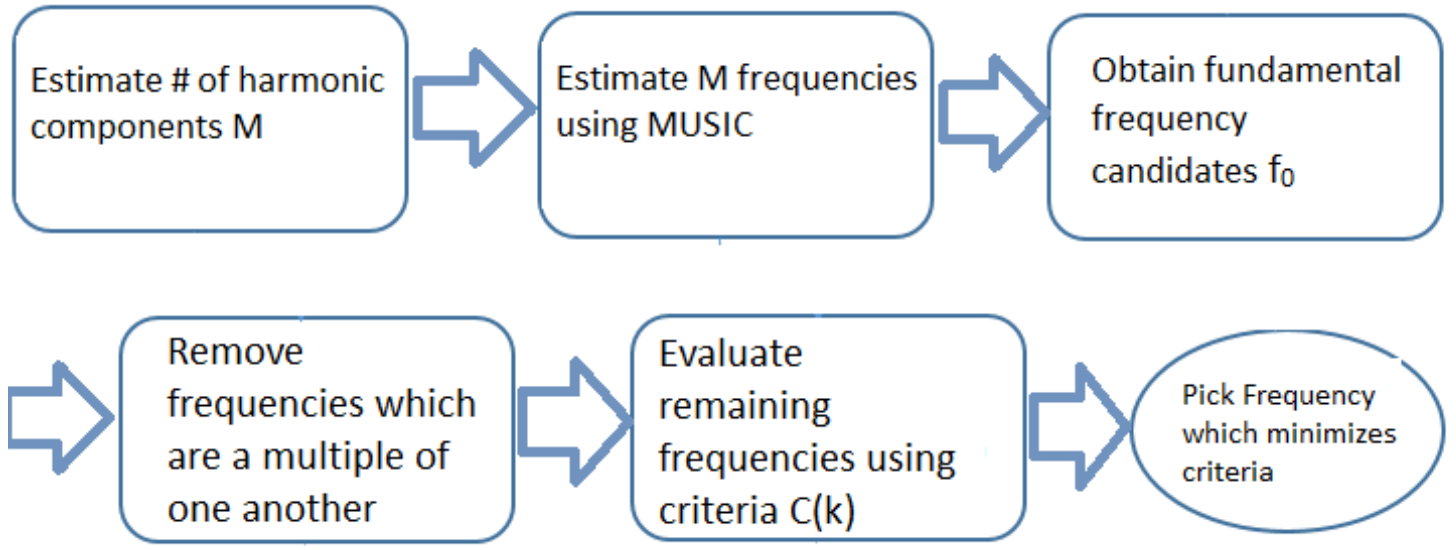

Figure 38: Steps for fundamental frequency estimation using MUSIC. 


\subsection{Performance Evaluation with Time Series Micro- Doppler Model}

In this section, the performance of the proposed fundamental frequency estimation techniques are compared to the popular existing methods in Section 5.2 using simulated micro-Doppler signal. The simple HERM line model devised in Chapter 3 is used to generate the simulated micro-Doppler signal for a single rotor drone with fundamental frequency of $80 \mathrm{~Hz}$ and five HERM lines. The parameters are shown in Table 12 and the spectrogram is shown in Figure 39.

Table 12: Single rotor HERM line model parameters.

\begin{tabular}{|l|l|}
\hline Parameter & Value \\
\hline$a_{0}$ & 1 \\
\hline$a_{n}$ & 0.1 \\
\hline$f_{c}$ & 80 \\
\hline$M$ & 5 \\
\hline $\mathrm{PRF}$ & $1.5 \mathrm{KHz}$ \\
\hline
\end{tabular}

Table 13: Effect of varying the number of harmonics summed up for the log harmonic summation method in the presence of $10 \mathrm{~dB}$ noise.

\begin{tabular}{|l|l|l|l|l|}
\hline M & 3 & 4 & 5 & 6 \\
\hline Accuracy & 0.92 & 0.99 & 1 & 0.98 \\
\hline
\end{tabular}

First, an experiment is conducted to study how varying the number of harmonic summed up affects the performance of the log harmonic summation method. Additive white Gaussian noise (AWGN) with a variance of $10 \mathrm{~dB}$ was added and 1000 fundamental frequency estimates were obtained using a non-overlapping window of 512 samples. Since $M=5$, the best performance should be obtained by summing up five harmonics. Table 13 shows the accuracy of the fundamental frequency estimate 


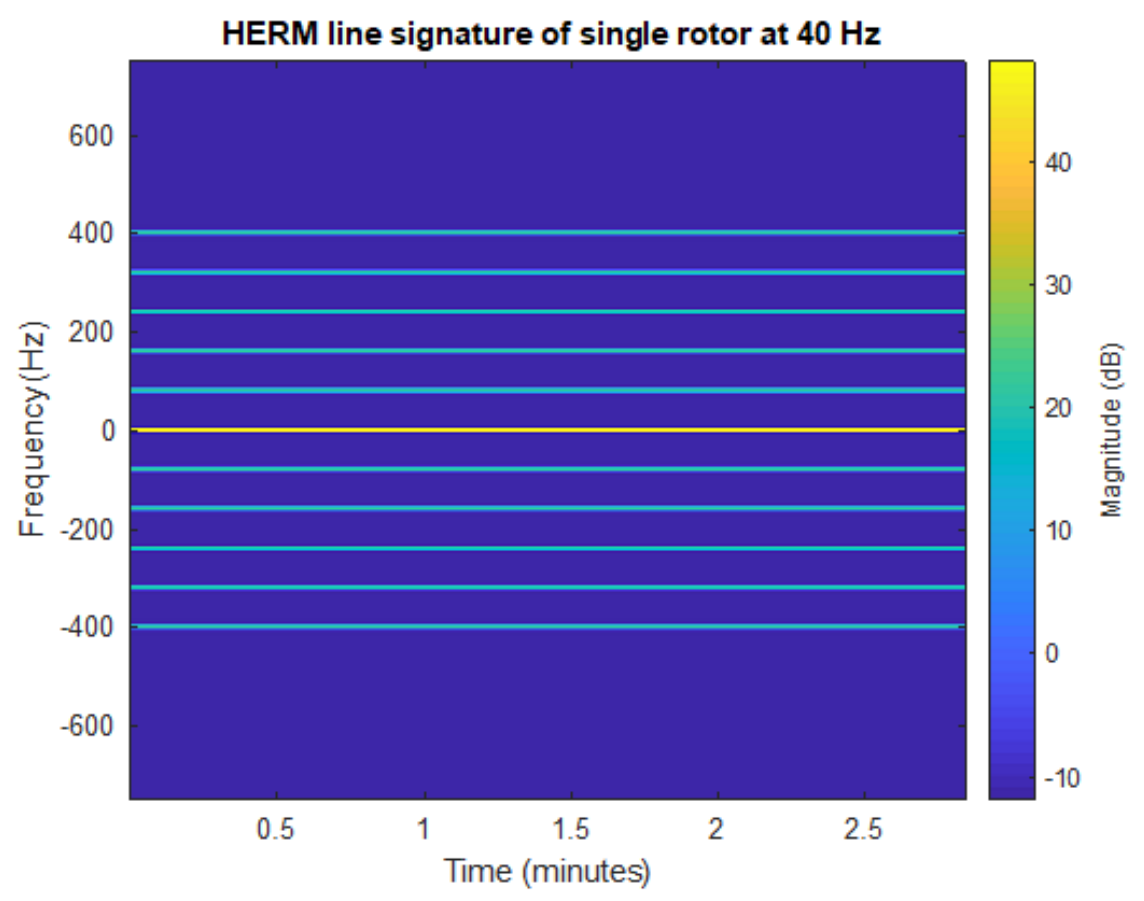

Figure 39: Simulated HERM line signatures.

for varying number of harmonics that were summed up. The best performance was achieved when the exact number of harmonic components was summed up. Summing up 1 less or 1 more harmonic did not significantly impact the performance.

For the rest of the results presented in this chapter, it is assumed that the number of harmonic components are known. The number of harmonic components can be estimated either using the parametric subspace dimension estimators or peak detection methods from the previous chapter.

A comparison of the four fundamental frequency estimation techniques in the presence of three different levels of AWGN noise $(10 \mathrm{~dB}, 7 \mathrm{~dB}$ and $5 \mathrm{~dB})$ is considered (three different SNR levels). Two metrics namely the MSE (Mean Squared Error) and the bias in the estimate are used to evaluate the techniques. The mathematical formula for the metrics are given below: 


$$
\begin{gathered}
\operatorname{MSE}(\hat{F})=E\left[(F-\hat{F})^{2}\right] \\
\operatorname{Bias}(\hat{F})=F-E(\hat{F})
\end{gathered}
$$

For each fundamental frequency estimation technique, the fundamental frequency estimates were limited from 30 to $300 \mathrm{~Hz}$. For all four estimation methods, a data segment of 512 samples $(0.33 \mathrm{~s})$ was used for the fundamental frequency estimate and 1000 iterations were conducted for each SNR level.

Table 14 shows the MSE and bias of all four methods at different SNR levels.

Table 14: Comparison of fundamental frequency estimate algorithms.

\begin{tabular}{|l|l|l|l|l|l|l|l|l|}
\hline \multirow{2}{*}{ SNR } & \multicolumn{2}{|l|}{$\begin{array}{l}\text { Log Harmonic } \\
\text { Summation }\end{array}$} & \multicolumn{2}{l|}{ Cepstrum } & \multicolumn{2}{l|}{$\begin{array}{l}\text { Cadence } \\
\text { Velocity }\end{array}$} & \multicolumn{2}{l|}{ MUSIC } \\
\cline { 2 - 10 } & MSE & Bias & MSE & Bias & MSE & Bias & MSE & Bias \\
\hline $5 \mathrm{~dB}$ & 436 & -1.57 & $5.3 \mathrm{e} 3$ & 35.76 & 625 & -13.14 & 582 & -1.51 \\
\hline $7 \mathrm{~dB}$ & 136 & -1.01 & $4.3 \mathrm{e} 3$ & 29.83 & 572 & -9.86 & 279 & -0.62 \\
\hline $10 \mathrm{~dB}$ & 7.36 & -0.18 & $3.21 \mathrm{e} 3$ & 22.5 & 341 & -2.16 & 31.3 & 0.11 \\
\hline
\end{tabular}

The log harmonic summation performed the best followed by MUSIC, cadence velocity and the cepstrum. Figure 40 shows the histogram distributions of fundamental frequency estimates at an SNR of $7 \mathrm{~dB}$. The cepstrum method is prone to large outliers which contributes to its large MSE. The cadence velocity method performed similarly to the cepstrum but did not have large outliers due to the STFT window length optimization method by Molchanov. However, underestimation is still an issue. 

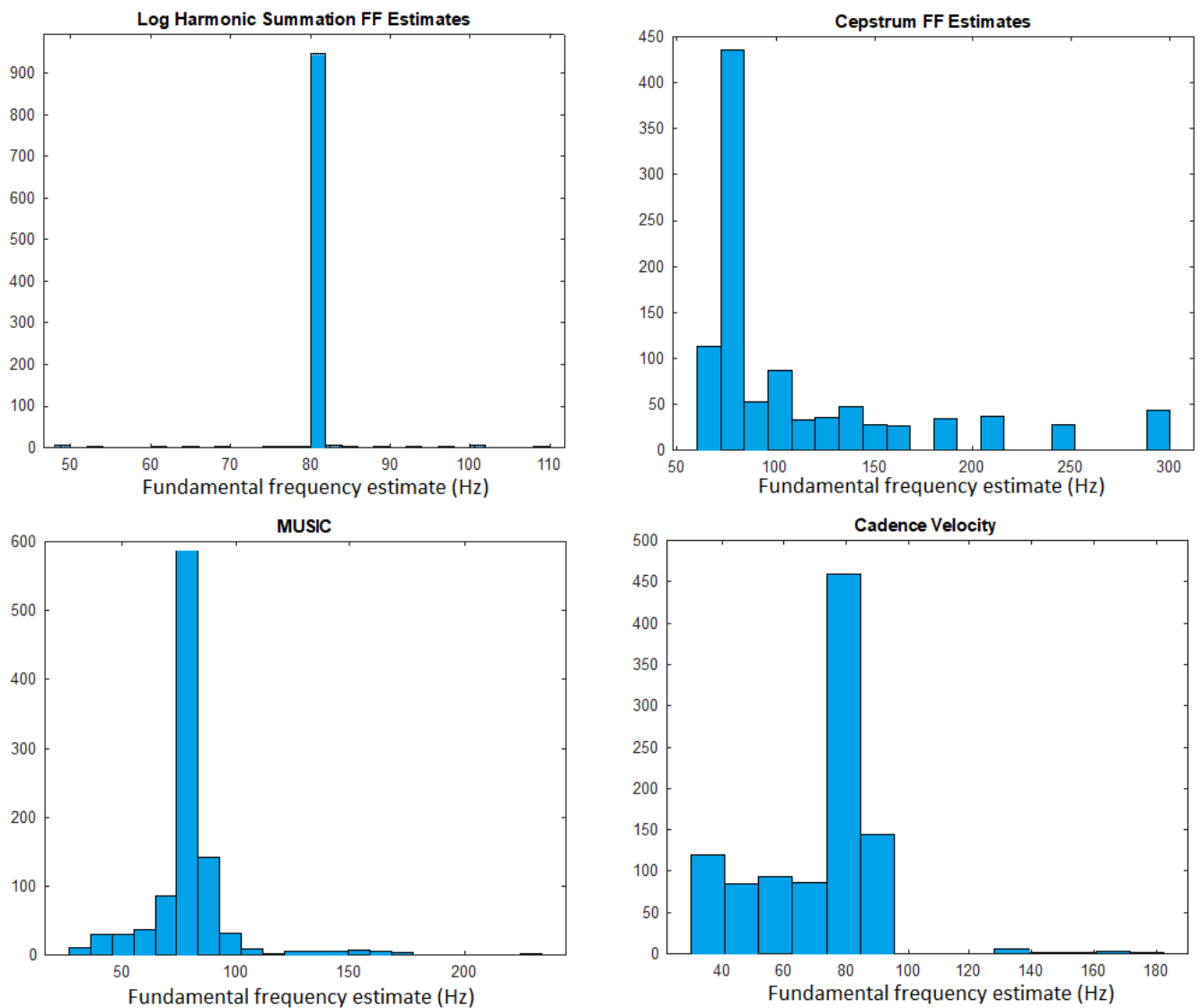

Figure 40: Histogram distribution of fundamental frequency estimation using the three methods on simulated data at an SNR of $7 \mathrm{~dB}$. 


\subsection{Performance Evaluation with Measured Micro- Doppler signal}

This section compares the performance of the fundamental frequency estimation techniques for real micro-Doppler captured from a single rotor micro-helicopter drone at $50 \mathrm{~cm}$ away from the Xethru X4 radar.

The HERM line signature in Figure 41 is almost similar to the one synthetically generated in Figure 39 but with some slight differences. Unlike the HERM lines in Figure 39, the HERM line components in Figure 41 do not have the same amplitude. The strongest HERM line component is not at the fundamental frequency so the selecting the frequency with the strongest peak will not suffice.

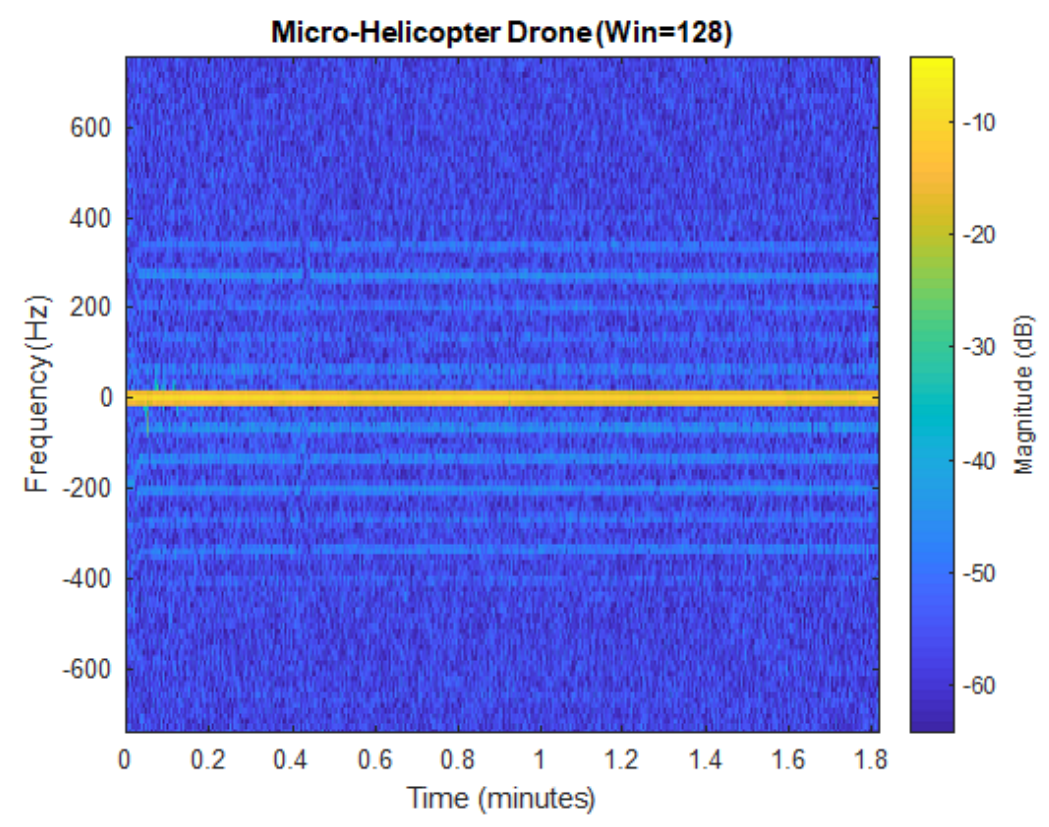

Figure 41: HERM line signature of single rotor micro-helicopter drone with rotor spinning at constant speed.

Figure 42 shows the histogram distributions of the estimated fundamental frequency 
using all four methods. In all methods, a non-overlapping data segment of length 512
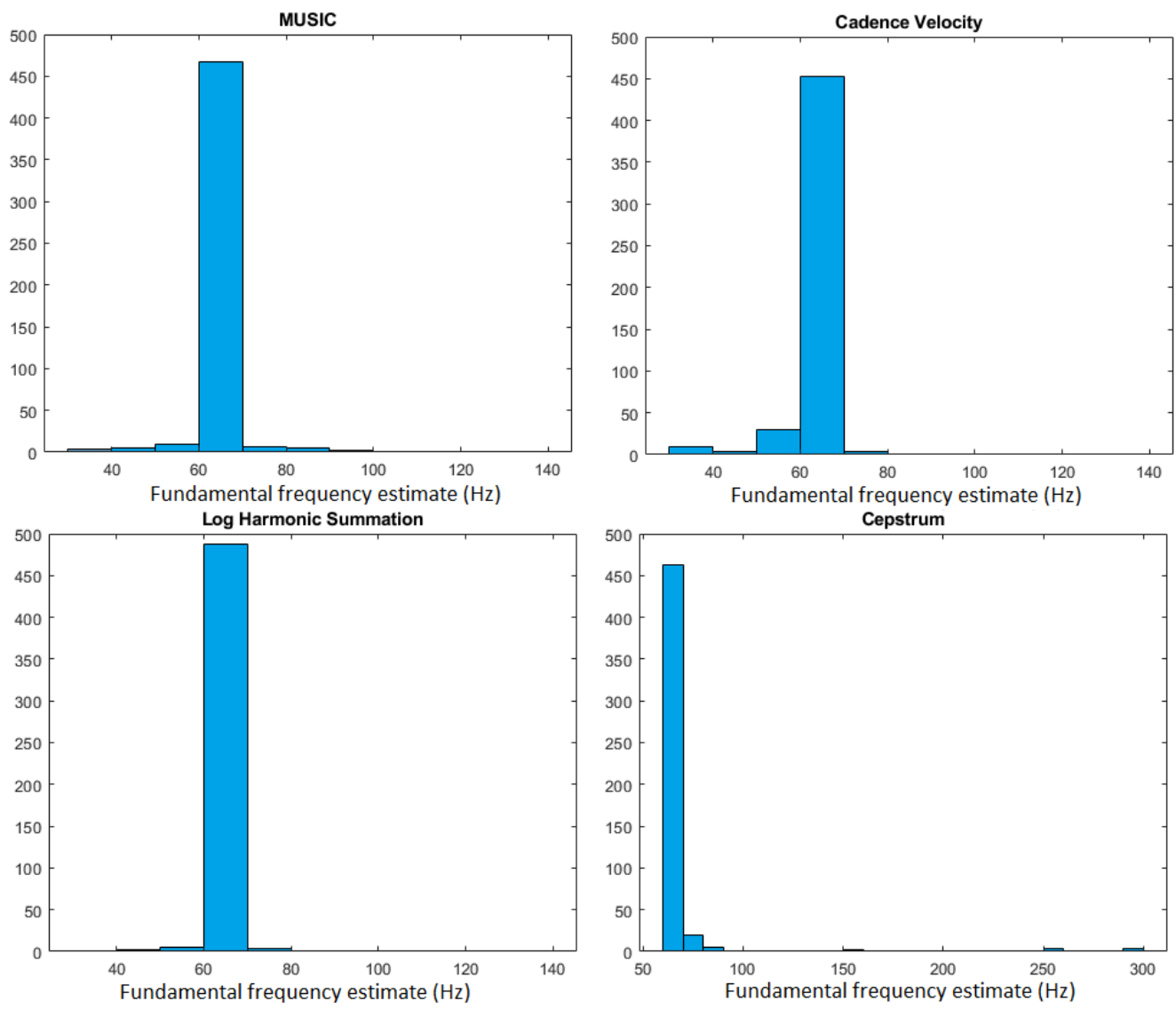

Figure 42: Histogram distribution for estimated fundamental frequency of single rotor micro-helicopter using non-overlapping window of 512 samples.

was used and 500 fundamental frequency estimates were made. The log harmonic summation method performs the best which matches the results in the previous section. The log harmonic summation had 475 estimates between the bins of $60-70 \mathrm{~Hz}$, while the MUSIC method had 467, the cepstrum had 458 and the cadence velocity method had 452 . 


\subsubsection{Short Observation Window}

A window of 512 samples $(0.33 \mathrm{~s})$ is used for the fundamental frequency estimate in the study conducted in the previous subsection. In this subsection, the effects of using a smaller window of 128 samples $(0.085 \mathrm{~s})$ is presented for all four algorithms. Figure 43 shows the histograms of the results. The MUSIC method actually performs better than the log harmonic summation with 408 estimates between 60 to $70 \mathrm{~Hz}$ while the log harmonic summation had 394 estimates between 60 to $70 \mathrm{~Hz}$. The MUSIC
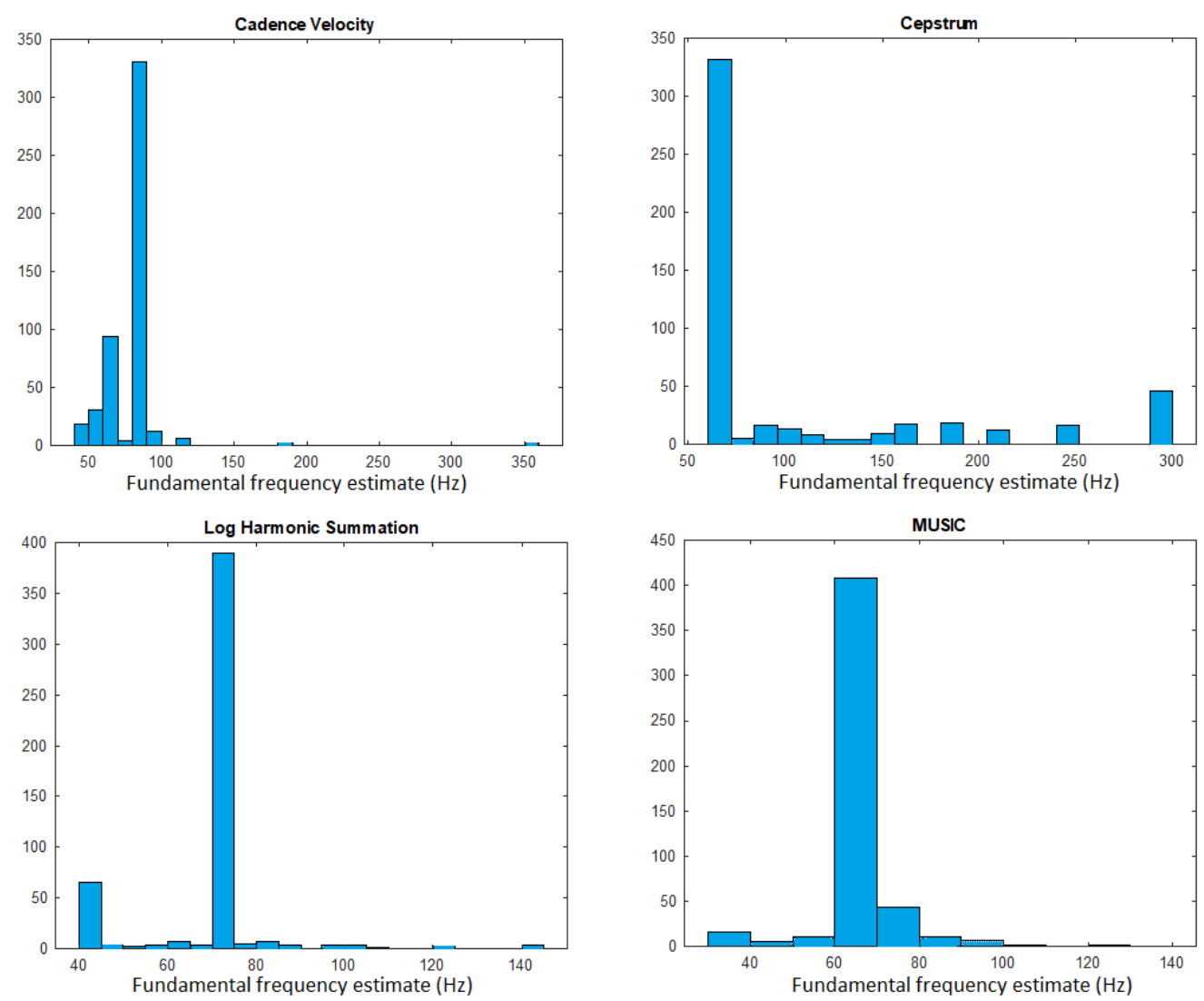

Figure 43: Fundamental frequency estimate using a window of 128 samples for single rotor micro-helicopter.

method also has the least variance as shown in the histograms. 
Table 15 shows a comparison of the true fundamental frequency estimate rate between 60-70 Hz as the observation window decreased from 512 samples (0.33 s) to 128 samples $(0.085 \mathrm{~s})$ for all 4 methods. The MUSIC method is most resilient to the short observation time.

Table 15: True fundamental frequency estimate rate.

\begin{tabular}{|l|l|l|}
\hline & 512 Samples $(0.33 \mathrm{~s})$ & 128 Samples $(0.085 \mathrm{~s})$ \\
\hline Log Harmonic Summation & 0.95 & 0.78 \\
\hline MUSIC & 0.934 & 0.82 \\
\hline Cepstrum & 0.91 & 0.66 \\
\hline Cadence Velocity & 0.9 & 0.66 \\
\hline
\end{tabular}

The main advantage of using MUSIC is for its superresolution capability meaning that it can resolve closely spaced frequencies without using a large window like Fourier techniques. This has applications for multi-rotor drones since they have very small frequency differences between their HERM lines due to their slightly different rotor speeds. An example is shown in Figure 44 for the flying DJI Spark quadcopter at 4 $m$ using the Ancortek radar. A feature that can be used to distinguish a multi-rotor drone is the minimum HERM line separation frequency.

As shown in Figure 45, using a FFT of 128 samples $(0.085 \mathrm{~s})$ is unable to resolve the closely spaced frequencies at around $400 \mathrm{~Hz}$. This is only resolvable using a longer FFT size of 256 samples $(0.17 \mathrm{~s})$. However, root music is able to estimate these closely spaced frequencies using a 40 by 40 covariance matrix generated using a smaller window of 128 samples $(0.085 \mathrm{~s})$ as shown by the red points. Training a classifier using MUSIC estimated frequencies may perform better than using Fourier generated HERM line spectrums for smaller window sizes. 


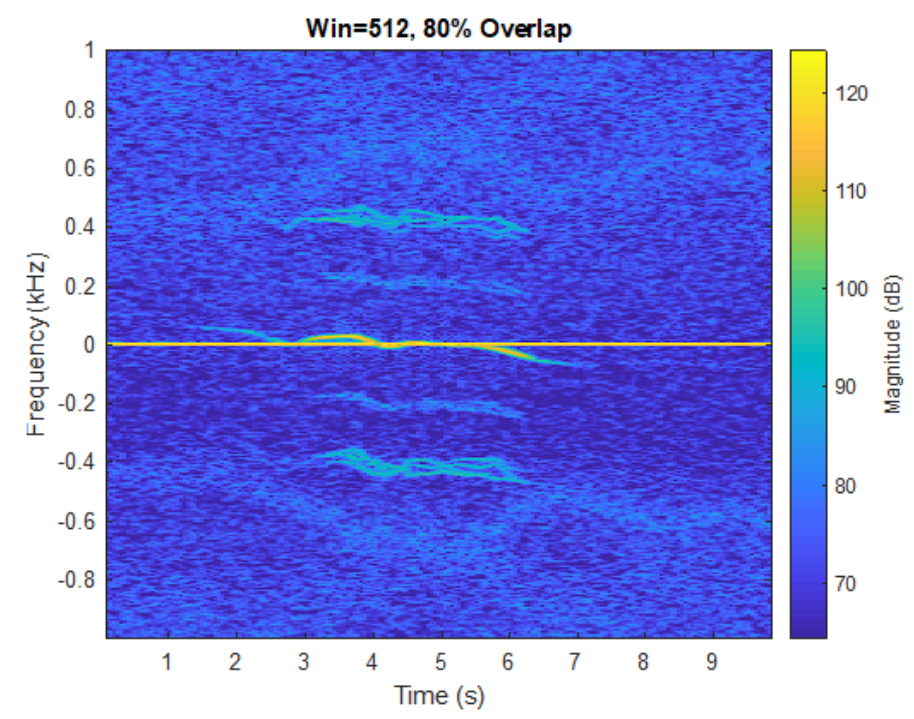

Figure 44: HERM line signature of flying DJI Spark quadcopter at $4 \mathrm{~m}$ generated using Hann window size of 512 samples with $80 \%$ overlap.
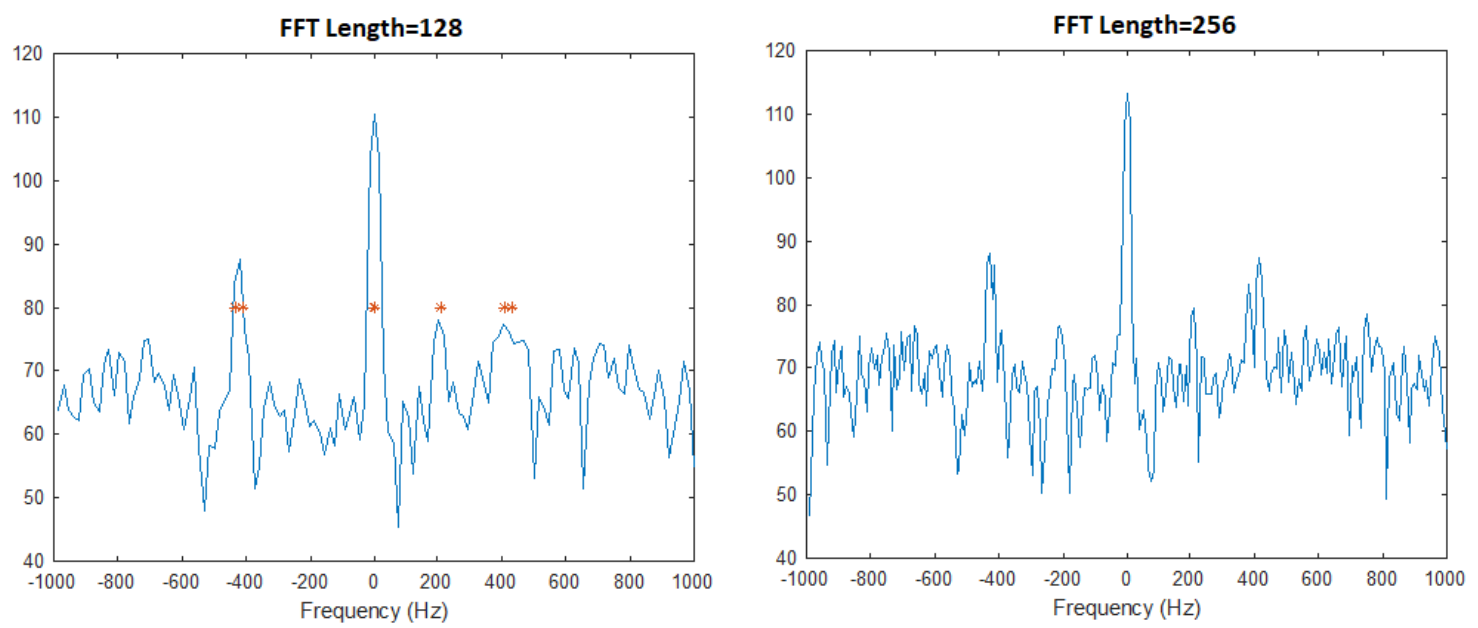

Figure 45: HERM line spectrum of flying DJI Spark quadcopter generated using a FFT of 128 samples (Left) and 256 samples (Right). The red points represent the frequencies estimated by root-MUSIC. 


\subsection{Drone Classification}

This section compares the performance of using the fundamental frequency against the number of HERM lines to classify three different classes of drones. The three different classes of the drones considered in this thesis are single rotor, multi-rotor and fixed wing drones. The number of HERM lines has the advantage of being invariant of the propeller rotation rate. According to the model by Martin and Mulgrew [16], the number of HERM lines is equal to the maximum micro-Doppler shift of the rotating propeller divided by the separation frequency,

$$
\begin{aligned}
N_{h} & =\frac{8 \pi l \Omega \cos (\phi)}{\lambda N \Omega} \\
& =\frac{8 \pi l \cos (\phi)}{\lambda N}
\end{aligned}
$$

where the HERM line separation frequency is $N \Omega$. Through simplification, the dependency on the propeller rotation rate $\Omega$ is removed. The number of HERM lines should stay constant unless there are additional propellers spinning at different rates such as for a multi-rotor drone $(C>1)$. Using the number of HERM lines to classify drones should perform better than using the fundamental frequency.

Figure 46 shows the HERM line spectrograms for three classes of drones, single rotor (Single), multi-rotor (Multi) and fixed wing (Fixed). All 3 datasets were from the Xethru X4 radar and all 3 drones were fixed on a stand at distances $<2 \mathrm{~m}$ away from the radar. The multi-rotor drone had its rotors spinning at varying speeds to emulate a maneuvering scenario while the other 2 drones had their rotors spinning at constant speed. The fixed wing drone had the fastest propeller rotation rate so it had the least amount of HERM lines and the largest fundamental frequency. The single rotor drone had the most HERM lines and the least fundamental frequency.

Two features were extracted, the number of HERM lines and the fundamental frequency using a non-overlapping window size of 128 samples $(0.085 \mathrm{~s})$. The number of HERM lines was estimated using the Radoi method and the fundamental frequency 


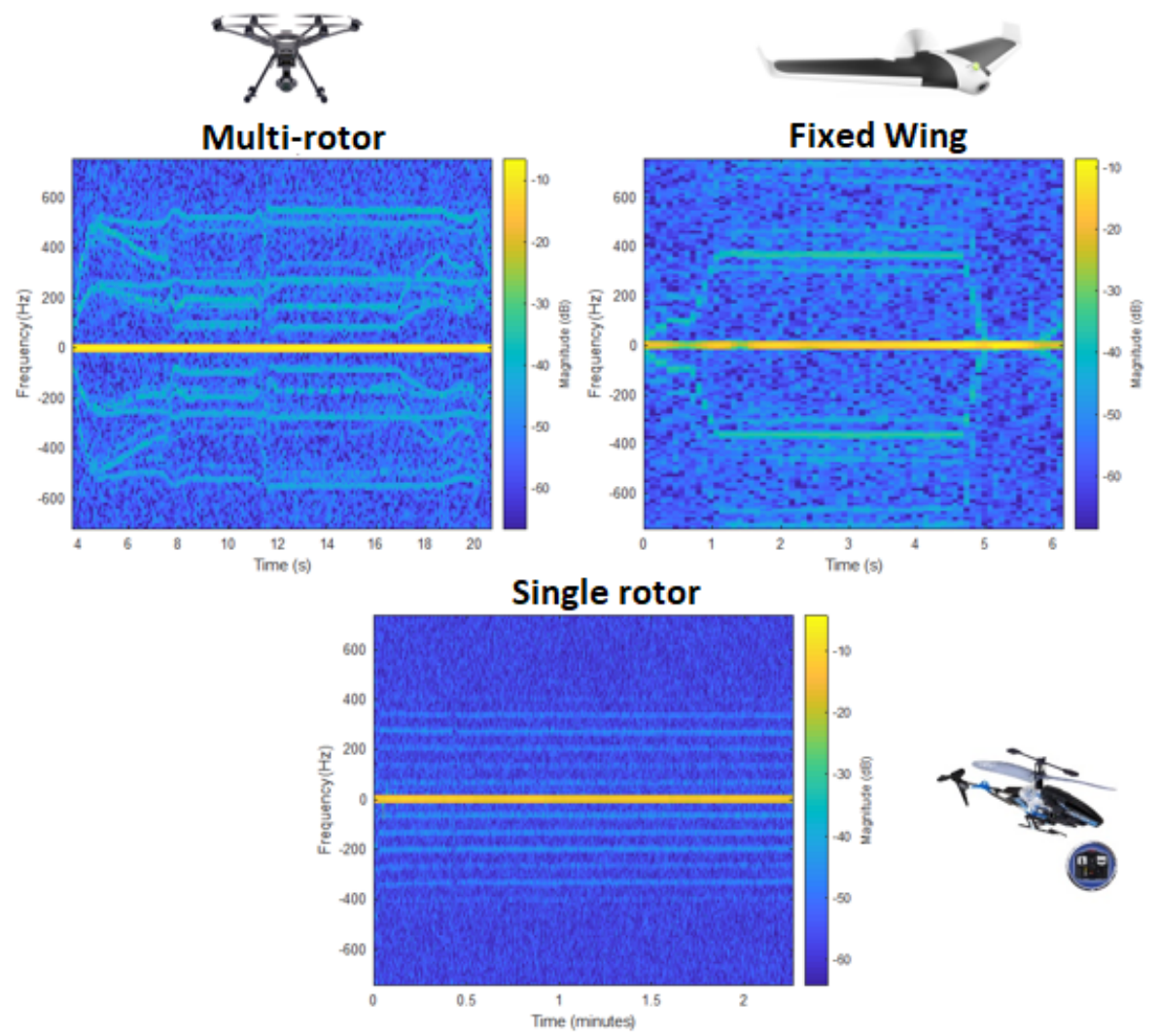

Figure 46: HERM line spectrogram comparison of three types of drones.

was estimated using the log harmonic summation. 125 data points were obtained for each drone. Figure 47 shows the histogram distribution of the two features. There is better separability between the three classes of drones using the number of HERM lines than the fundamental frequency.

Classification is done using a Naive Bayes classifier. Naive Bayes is a parametric technique-based classifier which assumes that all features are independent and uses the maximum posterior probability to make the classification decision.

$$
p(c \mid x)=\frac{p(c) p(x \mid c)}{p(x)}
$$

where $p(c)$ is the prior and $p(x \mid c)$ is the conditional probability of feature set $x$ in 

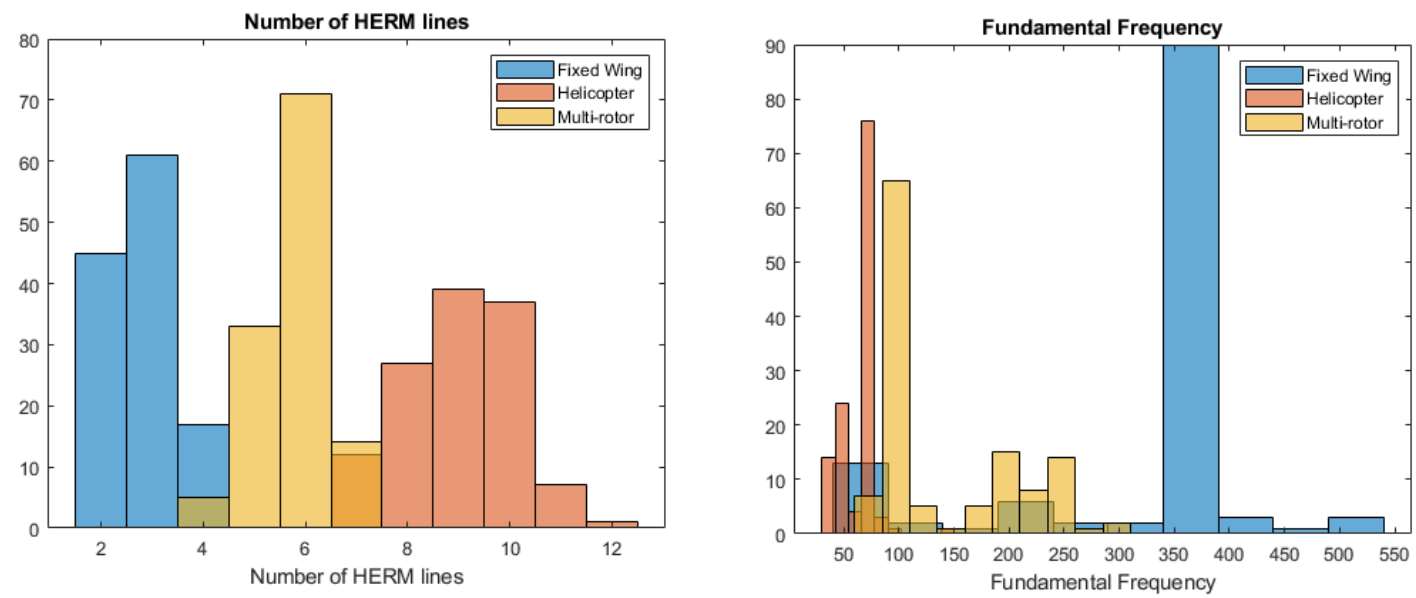

Figure 47: Histogram distribution of the number of HERM lines and the fundamental frequency for the three types of drones.

relation to the classes $c$. The features are assumed to fit to a Gaussian distribution.

The data is first partitioned into a training and validation set. $70 \%$ of the data is used for training and $30 \%$ is used for validation. Stratified sampling is used to ensure the number of samples per class is the same. Stratified sampling is just randomly sampling from each class so that the amount of samples per class is proportional to the original dataset. The validation data is then fed into the trained classifier to assess the performance. The process of randomly sampling data into training and validation dataset is repeated 100 times to evaluate the performance of the classifier. Table 16 shows the confusion matrix of using the number of HERM lines as a feature. In all the confusion matrices shown in this thesis, rows indicate predicted classes.

Using this feature, the probability of correctly distinguishing a fixed wing drone is $100 \%$, the single-rotor at $90.4 \%$ and the multi-rotor at $96.5 \%$. The worst performance is distinguishing a multi-rotor drone from a single-rotor drone at $90.4 \%$. This was because the additional propellers of the multi-rotor drone added extra harmonic components. 
Table 16: Confusion matrix for Naive Bayes classifier using number of harmonic components as a feature.

\begin{tabular}{|l|l|l|l|}
\hline Class & Single & Multi & Fixed \\
\hline Single & 90.4 & 9.6 & 0 \\
\hline Multi & 0 & 96.5 & 3.5 \\
\hline Fixed & 0 & 0 & 100 \\
\hline
\end{tabular}

On the other hand, using the fundamental frequency is able to distinguish singlerotor drones very well at $98 \%$ but did not perform well for multi-rotor and fixed wing drone as shown in Table 17. This is because the multi-rotor and fixed wing drone had varying rotor speeds. The fundamental frequency does not generalize well for varying rotor speeds. This demonstrates the superiority of using the number of HERM lines for classification of drones under maneuvering conditions.

Table 17: Confusion matrix for Naive Bayes classifier using fundamental frequency as a feature.

\begin{tabular}{|l|l|l|l|}
\hline Class & Single & Multi & Fixed \\
\hline Single & 98 & 2 & 0 \\
\hline Multi & 25 & 61 & 14 \\
\hline Fixed & 10 & 8 & 82 \\
\hline
\end{tabular}

When both features are used together, the probability of correctly distinguishing a single rotor drone increases to $99 \%$ but the probability of correctly distinguishing a fixed wing drone decreases to $98 \%$. This is shown in Table 18. The fundamental frequency is a good supplementary feature for single rotor drones since they have many HERM lines making accurate estimation of the total number of HERM lines difficult. 
Table 18: Confusion matrix for Naive Bayes classifier using both features.

\begin{tabular}{|l|l|l|l|}
\hline Class & Single & Multi & Fixed \\
\hline Single & 99 & 1 & 0 \\
\hline Multi & 1 & 96.5 & 2.5 \\
\hline Fixed & 0 & 2 & 98 \\
\hline
\end{tabular}

\subsection{Conclusion}

In this chapter, two methods of estimating the fundamental frequency $f_{c}$ and the number of fundamental frequencies $C$ using the HERM line spectrum are proposed. The first method uses a template matching filter to sum up the amplitudes of the harmonically spaced spectral peaks in the HERM line spectrum. The fundamental frequency is then selected based on the frequency which has the strongest harmonics. The second method first estimates the number of harmonic components using methods described in the previous chapter and then applies MUSIC to estimate the frequencies of the HERM line components. The fundamental frequency estimate is then determined based on the limited set of frequencies. In addition, popular methods in the literature like the cepstrum and the cadence velocity diagram were reviewed.

A comparison of these fundamental frequency estimation techniques was done using both simulated and real drone micro-Doppler signatures. Two metrics were evaluated, the robustness under noise and performance for short observation time windows. Through Monte Carlo simulations, the log harmonic summation was shown to be more robust under noise than the other methods using a window of 512 samples ( 0.33 s). However, the MUSIC method of estimating the fundamental frequency performed better than the Fourier-based techniques for short duration of data $(0.085 \mathrm{~s}$ instead of $0.33 \mathrm{~s}$ ) due to its superresolution capability.

In addition, MUSIC is shown to be able to estimate closely spaced fundamental 
frequencies better than Fourier-based techniques for smaller window lenghts of data. This has applications for distinguishing multi-rotor drones since they have very small differences between the HERM line components.

Finally, the classification performance using the number of HERM lines and the fundamental frequency for drone classification was demonstrated using a Naive Bayes classifier. Three classes of drones are considered: single rotor, multi-rotor and fixed wing. Classification using the fundamental frequency is only effective for the hovering case while the number of HERM lines generalizes better for varying rotor speeds. The overall accuracy of using the number of HERM lines is $95 \%$ while the accuracy is only $80 \%$ using the fundamental frequency. 


\section{Chapter 6}

\section{Conclusion}

The detection and classification of drones is an important and growing field of research due to the proliferation of small commercial UAVs. Radar is an attractive option since it has longer range than other methods. However, conventional radar techniques are inadequate for detecting small UAVs due to their small RCS, low speed and low altitude flight. The use of micro-Doppler features may aid in the detection and classification of these small targets. In this thesis, the problem of drone detection and classification using micro-Doppler was studied using off-the-shelf software defined radars.

In this thesis, an emphasis was put on developing methods for low PRF radars for longer range micro-Doppler drone detection/classification. As such, micro-Doppler analysis needs to be done using a longer coherent processing window encompassing at least one rotor rotation rate. This results in the analysis of HERM line signatures which consist of harmonic sidebands obtained by taking a long windowed STFT.

Experiments were conducted to study HERM line signatures of different drones at different conditions and ranges. Based on these results, a simple mathematical model 
based on a sum of weak sinusoids was developed to represent the micro-Doppler signal obtained from single and multi-rotor drones. This model performs well when the drone is flying approximately line of sight with the radar.

Based on this model, three features are identified to characterize rotary drone microDoppler: the number of HERM lines, the fundamental frequencies and the number of fundamental frequencies. Each propeller on the drone will generate its own set of HERM lines with fundamental frequency related to the propeller rotation rate.

Feature extraction using subspace-based parametric spectral estimation techniques were explored. The current state of art in HERM line feature extraction depends on Fourier-based techniques which suffer from resolution issues for small window lengths of data. Both information theoretic and empirical methods of estimating the signal subspace were used to estimate the number of HERM lines. It was found that classic information theoretic techniques like MDL and AIC underestimate the number of weak HERM line components. The empirical method by Radoi performed better and rivals spectrogram peak detection methods of estimating the number of HERM lines. After estimating the number of harmonic components, MUSIC can be applied to estimate the frequencies of the HERM line components. Root MUSIC was shown to be able to estimate closely spaced frequencies better than Fourier-based techniques for short windows of data. This is important for micro-Doppler analysis of multirotor drones since they have very small frequency deviations between their HERM line components due to their slightly different rotor speeds.

Finally, the classification performance of using these features were explored using Naïve Bayes to classify three different classes of drones: single rotor, multi-rotor and fixed wing. Using the fundamental frequency classifies well for hovering drones while using the number of HERM lines is invariant to the rotor rotation rate.

The contributions of this thesis is the development of a simple model to define the micro-Doppler signal from a rotary drone with three variables: the number of 
HERM lines, the propeller rotation rate and the number of rotors. Fourier-based and parametric spectral estimation techniques were applied to simulated and real micro-Doppler signatures to extract these features. Parametric spectral estimation techniques were shown to perform better than Fourier-based techniques of feature extraction for shorter duration of data. This work may be useful for the development of micro-Doppler based drone detection/classification systems.

\subsection{Limitations and Future Work}

The overarching problem in the research of micro-Doppler-based drone classification is the lack of representative data. Most of the data collected in this thesis were of stationary drones in an indoor lab environment. This is not representative of a real flying scenario where there are wind effects and clutter from trees. Future work will consist of collecting more realistic data in various flying conditions to validate the performance of these micro-Doppler techniques. With the collection of more data, the performance of the drone detection/classification techniques could be validated more thoroughly. For the Naive Bayes classifier, only 125 samples of each class were used. This also prevented the use of deep learning techniques.

The TI AWR1642 radar has MIMO capabilities but MIMO was not used in the experiments. MIMO utilizes multiple receive and transmit antennas to obtain better spatial resolution. This could be useful for dealing with swarms of small drones as well as pinpointing their exact location. In this thesis, only one drone was considered at a time. Future work could consist of detecting and distinguishing multiple drones using MIMO capabilities.

In this thesis, only micro-Doppler features were used to detect/classify drones. However, the use of micro-Doppler features may be limited in range by SNR. Data fusion with other radar track features such as flight altitude, Doppler may provide better 
results.

Range experiments were conducted to estimate the minimum SNR at which microDoppler techniques can be applied. However, since RCS measurements of the drone were not conducted, the validity of the results are not confirmed. Future work could consist of conducting RCS measurements in an anechoic chamber in order to provide more accurate results.

Birds may be wrongly considered as drones when they are far away. Drone detection and classification should be considered with birds as a possible class. Future work should be dedicated to modelling and classification of flying birds in conjunction with different types of drones.

In this thesis, only the STFT was considered for studying micro-Doppler signatures. The STFT utilizes a fixed window siz. Therefore, there is a trade-off between time and frequency resolution. Optimizing the window size is important for generating shortwindowed micro-Doppler spectrograms to observe the blade flashes clearly. Adaptive window sizes are also important for ensuring stationarity to optimize the parametric spectral estimation techniques. Future work will focus on applying adaptive signal processing techniques to optimize the window size. For instance, techniques of generating micro-Doppler signatures using the wavelet transform may be considered.

One of the major issues in the research of micro-Doppler-based techniques is that almost all the techniques in the literature have been done using offline processing. In this thesis, parametric spectral estimation techniques were shown to perform better than Fourier-based techniques for smaller observation windows. Smaller observation windows may potentially reduce the processing time and aid in the development of a real time radar drone ATR system. Future work may focus on integrating these techniques into a real radar system as well as taking into consideration of link budget concerns like the ADC quantization noise. 


\section{Bibliography}

[1] CBC news. (2016). "Fighter jets scrambled after drone spotted near ottawa airport," [Online]. Available: https : //www. cbc.ca/news/canada/ottawa/ drone-ottawa-airport-cf18s-fighters-scrambled-1.3621633.

[2] Transport Canada. (2020). "Flying your drone safely and legally," [Online]. Available: https ://tc . canada.ca/en/aviation/drone-safety/flyingyour-drone-safely-legally.

[3] J. Gundlach, Designing unmanned aircraft systems: A comprehensive guide. American Institute of Aeronautics and Astronautics (AIAA), 2014.

[4] Transport Canada. (2021). "Find your category of drone operation," [Online]. Available: https://tc.canada.ca/en/aviation/drone-safety/find-yourcategory-drone-operation.

[5] S. Rahman and D. A. Robertson, "In-flight RCS measurements of drones and birds at K-band and W-band," IET Radar, Sonar and Navigation, vol. 13, no. 2, pp. 300-309, Feb. 2019.

[6] F. Hoffmann, M. Ritchie, F. Fioranelli, A. Charlish, and H. Griffiths, "MicroDoppler based detection and tracking of UAVs with multistatic radar," in 2016 IEEE Radar Conference (RadarConf), 2016, pp. 1-6.

[7] B. Taha and A. Shoufan, "Machine learning-based drone detection and classification: State-of-the-art in research," IET Radar, Sonar and Navigation, vol. 12, pp. 138669-138682, Jun. 2018. 
[8] B. K. Kim, H. Kang, and S. Park, "Drone classification using convolutional neural networks with merged Doppler images," IEEE Geoscience and Remote Sensing Letters, vol. 14, no. 1, pp. 38-42, 2017. DOI: 10.1109/LGRS . 2016. 2624820 .

[9] S. Björklund and N. Wadströmer, "Target detection and classification of small drones by deep learning on radar micro-Doppler," in 2019 International Radar Conference (RADAR), 2019, pp. 1-6. DOI: 10.1109/RADAR41533. 2019.171294.

[10] R. I. A. Harmanny, J. J. M. de Wit, and G. P. Cabic, "Radar micro-Doppler feature extraction using the spectrogram and the cepstrogram," in Proceedings of 11th European Radar Conference, vol. 11, Oct. 2004, pp. 165-168.

[11] S. Rahman and D. A. Robertson, "Millimeter-wave micro-Doppler measurements of small UAVs," in Proc. SPIE 10188, Radar Sensor Technology XXI, 101880T, Anaheim, California, May 2017. DOI: 10.1117/12.2261942.

[12] R. M. O'Donnell. (2002). "The radar equation," [Online]. Available: https : / / www . ll . mit . edu / sites / default / files / outreach / doc / 2018-07 / lecture $\ \% 202$.pdf.

[13] G. M. Brooker, "Understanding millimetre wave FMCW radar," in International Conference on Sensing Technology, 2005.

[14] M. Jankiraman, FMCW radar design. Artech House, 2018.

[15] V. C. Chen, The micro-Doppler effect in radar. Artech House, 2011.

[16] J. Martin and B. Mulgrew, "Analysis of the theoretical radar return signal from aircraft propeller blades," in IEEE Radar Conference, Sep. 1990, pp. 569-572.

[17] J. Markow and A. Balleri, "Examination of drone micro-Doppler and JEM/HERM signatures," in 2020 IEEE Radar Conference (RadarConf20), 2020, pp. 1-6. DOI: 10.1109/RadarConf2043947.2020.9266342.

[18] Y. Cai, "Radar micro-Doppler patterns for drone characterization," M.S. thesis, Dept. Elec. Eng., Delft University of Technology, Netherlands, 2019. 
[19] P. Molchanov, "Radar target classification by micro-Doppler contributions," Ph.D. dissertation, Dept. Sig. Proc., Tampere University of Technology, Finland, 2014.

[20] P. Zhang, L. Yang, G. Chen, and G. Li, "Classification of drones based on micro-Doppler signatures with dual-band radar sensors," in 2017 Progress in Electromagnetics Research Symposium - Fall (PIERS - FALL), 2017, pp. 638643. DOI: 10.1109/PIERS-FALL. 2017.8293214.

[21] A. K. Seifert, L. Schäfer, M. G. Amin, and A. M. Zoubir, "Subspace classification of human gait using radar micro-Doppler signatures," in 2018 26th European Signal Processing Conference (EUSIPCO), 2018, pp. 311-315. DOI: 10.23919/EUSIPCO.2018.8553592.

[22] W. Zhang and G. Li, "Detection of multiple micro-drones via cadence velocity diagram analysis," Electronic Letters, vol. 54, no. 7, pp. 441-443, 2018.

[23] M. Noll, "Cepstrum pitch determination," The Journal of the Acoustical Society of America, vol. 41, no. 2, pp. 293-309, 1967.

[24] L. Fuhrmann, O. Biallawons, J. Klare, R. Panhuber, R. Klenke, and J. Ender, "Micro-Doppler analysis and classification of UAVs at Ka band," in 2017 18th International Radar Symposium (IRS), 2017, pp. 1-9.

[25] J. S. Patel, C. Al-Ameri, F. Fioranelli, and D. Anderson, "Multi-time frequency analysis and classification of a micro-drone carrying payloads using multistatic radar," The Journal of Engineering, vol. 2019, no. 20, pp. 7047-7051, 2019. DOI: 10.1049/joe.2019.0551.

[26] F. Fioranelli, M. Ritchie, H. Griffiths, and H. Borrion, "Classification of loaded/ unloaded micro-drones using multistatic radar," Electronic Letters, vol. 51, no. 22, pp. 1813-1815, Oct. 2015.

[27] Y. Cai, O. Krasnov, and A. Yarovoy, "Radar recognition of multi-propeller drones using micro-Doppler linear spectra," in Proc. 16th European Radar Conference, Paris, France, Oct. 2019, pp. 185-188. 
[28] J. Gérard, J. Tomasik, C. Morisseau, A. Rimmel, and G. Vieillard, "MicroDoppler signal representation for drone classification by deep learning," in 2020 28th European Signal Processing Conference (EUSIPCO), 2021, pp. 1561-1565. DOI: 10.23919/Eusipco47968.2020.9287525.

[29] A. Brewster and A. Balleri, "Extraction and analysis of micro-Doppler signatures by the empirical mode decomposition," in 2015 IEEE Radar Conference (RadarCon), 2015, pp. 947-951.

[30] B. Oh, X. Guo, F. Wan, K. Toh, and Z. Lin, "Micro-Doppler mini-uav classification using empirical-mode decomposition features," IEEE Geoscience and Remote Sensing Letters, vol. 15, no. 2, pp. 227-231, 2018.

[31] M. Jahangir, B. I. Ahmad, and C. J. Baker, "Robust drone classification using two-stage decision trees and results from SESAR SAFIR trials," in 2020 IEEE International Radar Conference (RADAR), 2020, pp. 636-641.

[32] J. S. Patel, F. Fioranelli, and D. Anderson, "Review of radar classification and RCS characterisation techniques for small UAVs or drones," IET Radar, Sonar and Navigation, vol. 12, no. 9, pp. 911-919, Jun. 2018.

[33] J. Martin and B. Mulgrew, "Analysis of the effects of blade pitch on the radar return signal from rotating aircraft blades," in 92 International Conference on Radar, 1992, pp. 446-449.

[34] J. J. M. de Wit, R. I. A. Harmanny, and P. Molchanov, "Radar micro-Doppler feature extraction using the singular value decomposition," in 2014 International Radar Conference, 2014, pp. 1-6.

[35] M. Wax and T. Kailath, "Detection of signals by information theoretic criteria," IEEE Transactions on Acoustics, Speech, and Signal Processing, vol. 33, no. 2, pp. 387-392, 1985. DOI: 10.1109/TASSP. 1985.1164557.

[36] H. Akaike, "A new look at the statistical model identification," IEEE Transactions on Automatic Control, vol. 19, no. 6, pp. 716-723, 1974. DOI: 10.1109/ TAC.1974.1100705. 
[37] J. Rissanen, "Modeling by shortest data description," Automatica, vol. 14, no. 5, 465-471, Sep. 1978.

[38] G. Schwarz, "Estimating the dimension of a model," The Annals of Statistics, vol. 6, no. 2, pp. 461-464, 1978.

[39] C. Bennett, M. Jahangir, F. Fioranelli, B. Ahmad, and J. L. Kernec, "Use of symmetrical peak extraction in drone micro-Doppler classification for staring radar," in 2020 IEEE Radar Conference (RadarConf20), 2020, pp. 1-6.

[40] C. Clemente and J. J. Soraghan, "Application of the singular spectrum analysis for extraction of micro-Doppler signature of helicopters," in IET International Conference on Radar Systems (Radar 2012), 2012, pp. 1-5.

[41] Q. André and E. Radoi, "A new method for estimating the number of harmonic components in noise with application in high resolution radar," EURASIP Journal on Advances in Signal Processing, vol. 2004, pp. 1-12, Jul. 2004. DOI: 10. 1155/S1110865704401097.

[42] A. Oppenheim and R. Schafer, "From frequency to quefrency: A history of the cepstrum," IEEE Signal Processing Magazine, vol. 21, pp. 95 -106, Oct. 2004. DOI: $10.1109 /$ MSP . 2004.1328092.

[43] P. Molchanov, J. Astola, K. Egiazarian, and A. Totsky, "On micro-Doppler period estimation," in Proceedings - 19th International Conference on Control Systems and Computer Science, CSCS 2013, May 2013, pp. 325-330, ISBN: 9781-4673-6140-8. DOI: 10.1109/CSCS.2013.80.

[44] D. J. Hermes, "Measurement of pitch by subharmonic summation," Journal of the Acoustical Society of America, vol. 83, pp. 257-264, 1988.

[45] J. C. Brown, "Calculation of a constant Q spectral transform," The Journal of the Acoustical Society of America, no. 89, pp. 425-434, 1991.

[46] Y. Kim, M. Nazaroff, and D. Oh, "Extraction of micro-Doppler characteristics of drones using high-resolution time-frequency transforms," Microwave and Optical Technology Letters, vol. 60, no. 12, pp. 2949-2954, 2018. DOI: https:// doi .org/10.1002/mop. 31408. eprint: https ://onlinelibrary . wiley .com/ 
doi/pdf/10.1002/mop.31408. [Online]. Available: https://onlinelibrary . wiley.com/doi/abs/10.1002/mop. 31408 .

[47] B. D. Rao and K. V. S. Hari, "Performance analysis of root-music," IEEE Transactions on Acoustics, Speech, and Signal Processing, vol. 37, no. 12, pp. 19391949, 1989. DOI: $10.1109 / 29.45540$.

[48] C. Lovescu and S. Rao. (2017). "The fundamentals of millimeter wave sensors," [Online]. Available: http://www.ti.com/lit/wp/spyy005/spyy005.pdf.

[49] J. McCorkle. (2002). "Why such uproar over ultrawideband?" [Online]. Available: https://www. eetimes.com/document.asp?doc_id=1277563.

[50] Novelda AS, Xethru $x 4$ radar user guide, Novelda, 2018.

[51]—, X4m300 datasheet, Novelda, 2018.

[52] V. Dham. (2017). "Programming chirp parameters in TI radar devices," [Online]. Available: http://www.ti.com/lit/an/swra553/swra553.pdf.

[53] Ancortek Inc. (2019). "Who we are," [Online]. Available: https://ancortek. com/our-company.

[54] D. De Quevedo, F. I. Urzaiz, J. G. Menoyo, and A. A. López, "Drone detection and RCS measurements with ubiquitous radar," in 2018 International Conference on Radar (RADAR), 2018, pp. 1-6. DOI: 10.1109/RADAR.2018.8557320. 


\section{Appendix A}

\section{FMCW radar theory}

\section{A.1 FMCW Radar}

Unlike traditional pulsed radar that sends out pulses and listens to reflections, a CW (Continuous Wave) radar sends out a continuous sinusoidal stream of radio energy. The detection of moving objects can then be done using the Doppler shift between the transmitted and received signal Since the radar is constantly transmitting, it utilizes less transmit power than pulsed radars to achieve the same detection range. This is important for drone detection as more transmit power is required to detect these small targets at long ranges. However, pure CW radars cannot measure range since there is no way of timestamping the received signal.

In order to measure range, the $\mathrm{CW}$ signal needs to be modulated. A FMCW radar uses a linearly frequency modulated signal called a chirp. A chirp is a periodic sawtooth waveform which increases in frequency as time increases as shown in Figure 48. 

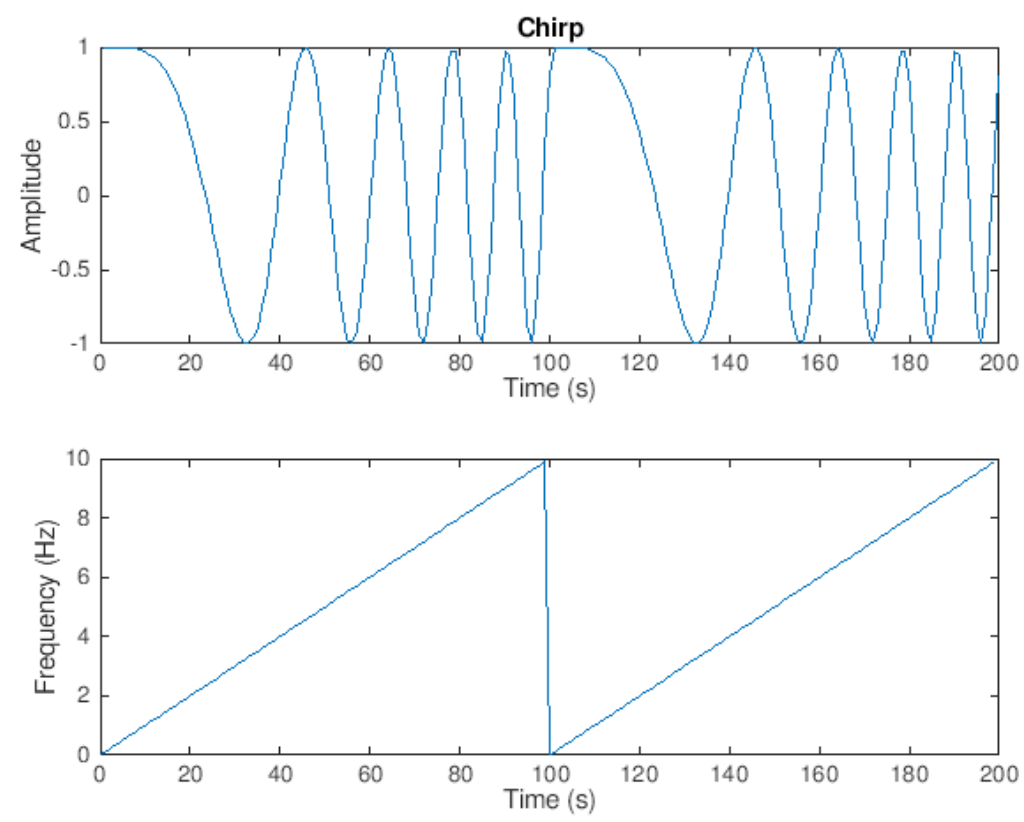

Figure 48: Chirp waveform in time and frequency domain

FMCW is able to measure distance and speed by comparing the frequency and phase difference between the transmitted and reflected chirp. This is done by mixing the two chirps to form a beat frequency. Figure 49 shows a block diagram of the FMCW radar. The chirp is generated by the synth and sent to the transmitter. The received signal and the transmitted signal is mixed together to form the IF (instantaneous frequency) signal.

The mixing of the transmitted and received signals can be proven mathematically as shown below. The equation for a linear chirp is:

$$
w_{t x}(t)=A \cos \left(\omega t+\frac{2 \pi K t^{2}}{2}\right)
$$

where $\mathrm{K}$ is the slope of the chirp waveform given by

$$
K=\frac{B}{T}
$$

where $\mathrm{B}$ is the bandwidth and $\mathrm{T}$ is the period of the chirp. 


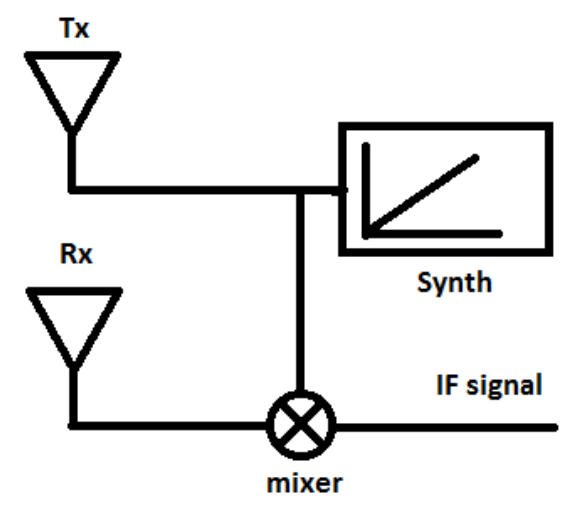

Figure 49: Block diagram of FMCW radar [48]

The time delay between the received and transmitted chirp is

$$
\tau=\frac{2 d}{c}
$$

where $\mathrm{d}$ is the one-way distance to the target and $\mathrm{c}$ is the speed of light. Thus the received signal will be shifted from the transmitted signal by $\tau$.

$$
w_{r x}(t)=A \cos \left(\omega(t-\tau)+\frac{2 \pi K}{2}(t-\tau)^{2}\right)
$$

Multiplying Equation 4 and 3 results in

$$
\begin{aligned}
I(t) & =w_{r x} w_{t x} \\
& =\frac{A^{2}}{2}\left[\operatorname { c o s } \left(\omega(t-\tau)+\frac{2 \pi K}{2}(t-\tau)^{2}-\left(\omega t+\frac{2 \pi K t^{2}}{2}\right)+\cos (\omega(t-\tau)\right.\right. \\
& \left.\left.+\frac{2 \pi K}{2}(t-\tau)^{2}+\left(\omega t+\frac{2 \pi K t^{2}}{2}\right)\right)\right] \\
& =\frac{A^{2}}{2}\left[\cos \left(\omega \tau+2 \pi K t \tau-\frac{2 \pi K \tau^{2}}{2}\right)+\cos \left(2 \omega t-\omega \tau+2 \pi\left(K t^{2}-K t \tau+\frac{K \tau^{2}}{2}\right)\right)\right]
\end{aligned}
$$

The first term represents the beat signal and the second represents a harmonic at double the frequency that is filtered out with a low pass filter. Thus the filtered IF signal is:

$$
I(t)=\frac{A^{2}}{2} \cos \left(\omega \tau+2 \pi K t \tau-\frac{2 \pi K \tau^{2}}{2}\right)
$$


The instantaneous beat frequency can be found by differentiating the beat signal [13].

$$
f_{b}=\frac{1}{2 \pi} \frac{d}{d t}\left[\omega \tau+2 \pi K t \tau-\pi K \tau^{2}\right]=K \tau
$$

Therefore the beat frequency is proportional to the time delay between the received and transmitted chirp in Equation 74. The range can be extracted using

$$
d=\frac{c f_{b}}{2 K}
$$

Figure 50 shows a visual representation of the transmitted, reflected and the resulting beat frequency. The instantaneous beat frequency is found by subtracting the transmitted and reflected signals or by using Equation 6. The Doppler shift is shown in the received chirp as a frequency shift from the carrier frequency.

The beat frequency could also be found by taking the FFT of the IF signal. This would resolve in multiple beat frequency peaks that correspond to different objects at different distances. According to Fourier transform theory, the width of the frequency lobes are inversely proportional to the length of the observational window[48]. Therefore, two IF signal tones could be distinguished if the difference between them was more than $\frac{1}{T_{c}}[48]$. Using Equation 7 , the range resolution is denoted by

$$
\begin{aligned}
\Delta d & =\frac{c f_{b}}{2 K} \\
& =\frac{c}{2 K T_{c}} \\
& =\frac{c}{2 B}
\end{aligned}
$$

Thus, the range resolution is dependent on the chirp bandwidth.

In the case where an object is moving, the time difference between the received and 

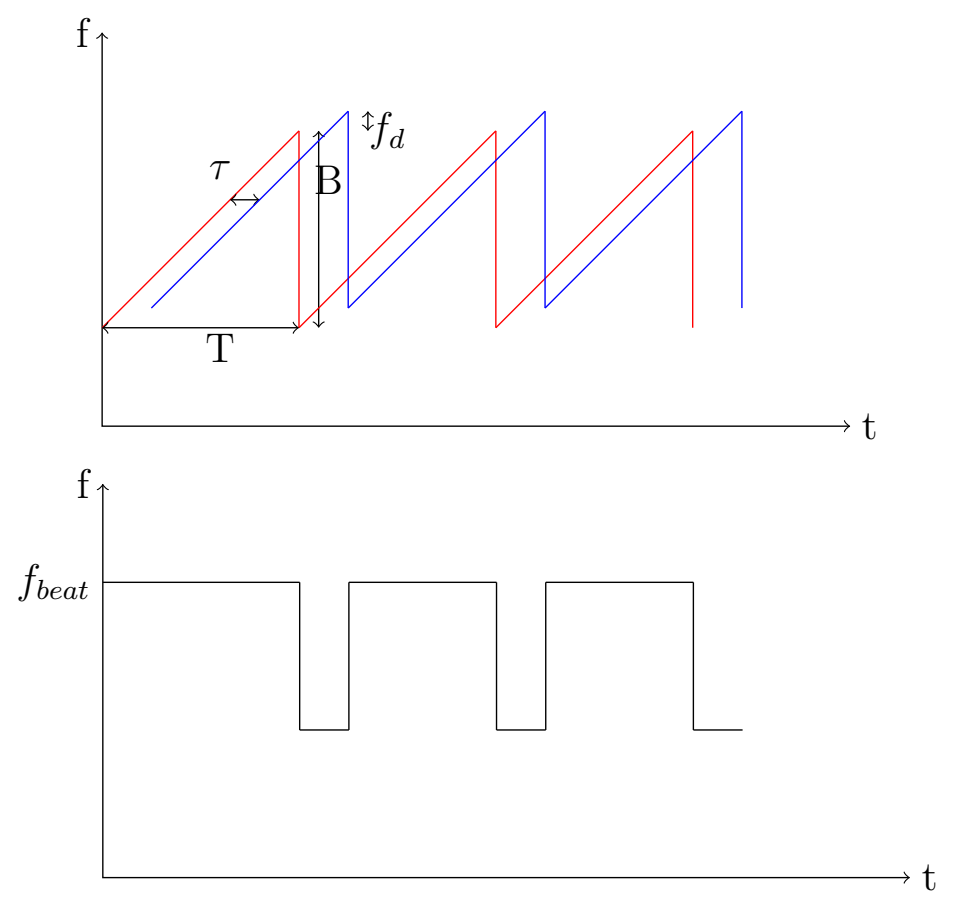

Figure 50: (Top) The red chirp represents the transmitted wave while the blue chirp represents the received wave. The mixed signal is shown below showing the beat frequency. The doppler shift denoted as $f_{d}$

transmitted chirp varies with time. Thus Equation 3 becomes:

$$
\begin{aligned}
\tau & =\frac{2(d+v t)}{c} \\
& =\tau_{0}+\frac{2 v t}{c}
\end{aligned}
$$

Substituting this into Equation 6 results in:

$$
\begin{aligned}
I(t) & =\frac{A^{2}}{2}\left[\cos \left(\omega\left(\tau_{0}+\frac{2 v t}{c}\right)+2 \pi K t\left(\tau_{0}+\frac{2 v t}{c}\right)-\frac{2 \pi K\left(\tau_{0}+\frac{2 v t}{c}\right)^{2}}{2}\right)\right. \\
& =\frac{A^{2}}{2}\left[\operatorname { c o s } \left(2 \pi f_{c}\left(\tau_{0}+\frac{2 v t}{c}\right)+2 \pi K t\left(\tau_{0}+\frac{2 v t}{c}\right)-\pi K\left(\tau_{0}^{2}+\frac{4 v t T_{0}}{c}+\left(\frac{2 v t T_{0}}{c}\right)^{2}\right)\right.\right.
\end{aligned}
$$


Since $\mathrm{t}$ and $T_{0}$ are really short durations, the terms where they are divided by the speed of light can be neglected. Thus Equation 11 is simplified as:

$$
I(t)=\frac{A^{2}}{2}\left[\cos \left(2 \pi f_{c}\left(\tau_{0}+2 \pi K t \tau_{0}\right)+\frac{4 \pi f_{c} v t}{c}\right)\right)
$$

Velocity can be determined using the phase difference between chirps. The phase difference term in Equation 12 is denoted by

$$
\phi=\frac{4 \pi v t}{\lambda}
$$

and the velocity is derived as:

$$
v=\frac{\lambda \phi}{4 \pi t}
$$

Looking at Equation 14, velocity will only be unambiguous if the phase is $<\pi$. Thus the maximum resolvable velocity is:

$$
V_{\max }=\frac{\lambda}{4 T_{c}}
$$

Velocity can also be found by using the 2D Fourier transform method where a second FFT is performed on the chirps for each range bin over time. This would resolve in separate velocity peaks for each moving object in the range bin. Thus velocity resolution would increase with more chirps. The equation for velocity resolution is:

$$
\Delta v=\frac{\lambda}{2 N T_{c}}
$$

where $\mathrm{N}$ is the number of chirps in a frame. The TI FMCW radar sends out a pulse of chirps in a frame. Figure 51 shows the process to generate range-Doppler plot by the 2D FFT method [48].

The maximum detectable range for a FMCW radar is limited by the maximum beat frequency. Recall for a FMCW radar the range is determined by:

$$
d=\frac{c f_{b}}{2 K}
$$



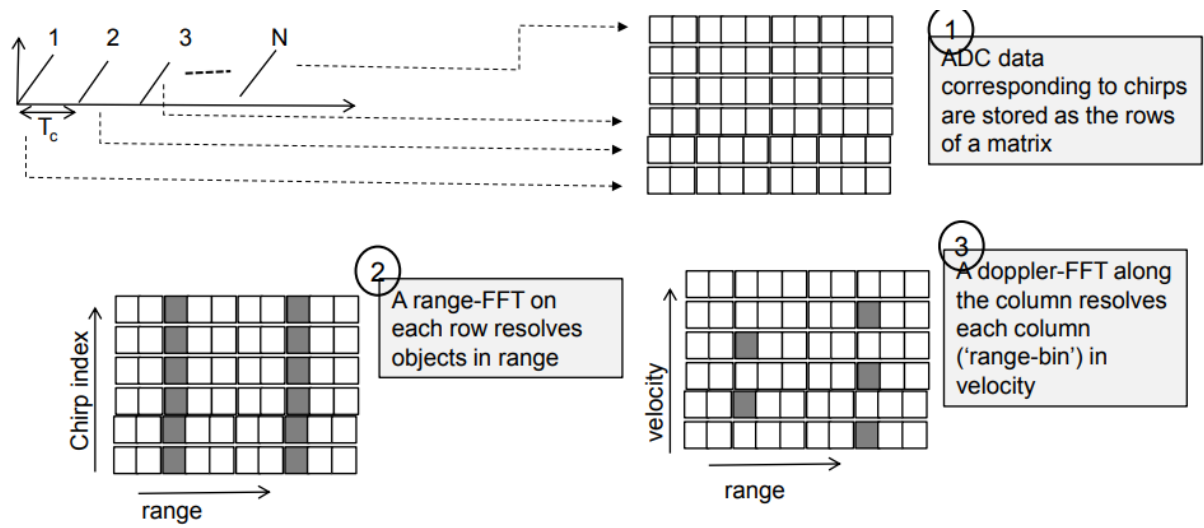

Figure 51: 2D FFT processing of FMCW radar [48]

Since the received chirp gets digitized by a low pass filter and an ADC, the maximum beat frequency is ultimately limited by the ADC sampling rate. The maximum beat frequency must satisfy the Nyquist theorem as shown in the Equation below.

$$
f_{\text {bmax }}=\frac{\text { ADCsamplerate }}{2}
$$

This is also known as the IF bandwidth. Typically, in FMCW radar design, the IF bandwidth is fixed since it depends on the ADC sampling rate and the chirp bandwidth is varied to change the maximum range. This will also coarsen the range resolution. 


\section{Appendix B}

\section{Radars used in this thesis}

\section{B.1 Xethru X4 UWB radar}

The Novelda X4 radar is an ultra-wideband (UWB) pulsed radar operating at 7.29 $\mathrm{GHz}$ designed for occupancy sensing and respiration monitoring purposes.

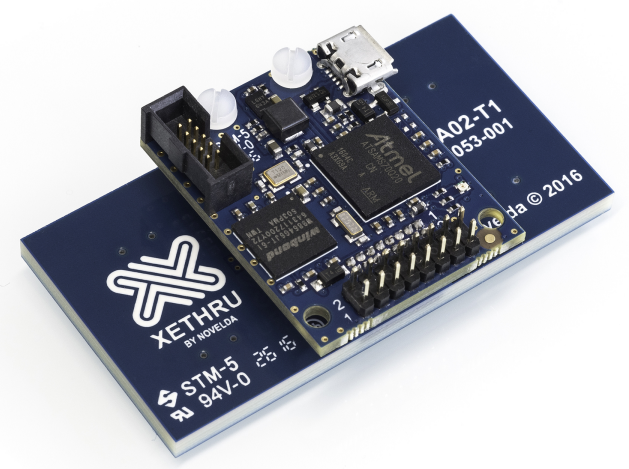

Figure 52: Xethru X4 Radar. 
UWB technology is characterized by its low power and large bandwidth for short range purposes [49]. Its low power allows it to occupy large bandwidth without interfering with other users. In order to have a large bandwidth, the pulse duration is very short, as a consequence the range resolution is very fine $(5.25 \mathrm{~cm})$. Although the PRF of this radar is very high ( $15.1875 \mathrm{MHz})$, the actual sample rate in slow time is actually very low due to the way a radar frame is generated. Because the UWB radar pulse has such a wide bandwidth, conventional match filtering techniques cannot be used since the reflected pulse will be distorted after reflecting off the environment. Instead, a radar frame is generated by coherently integrating multiple reflected pulses. Assuming that the target does not move within the coherent integration time, the resulting radar frame will contain a strong peak at the target range bin. Figure 53 shows an example of a radar frame and the downsampled radar frame by a factor of 8 [50]. The frame generation method currently used in the Novelda X4 UWB radar is
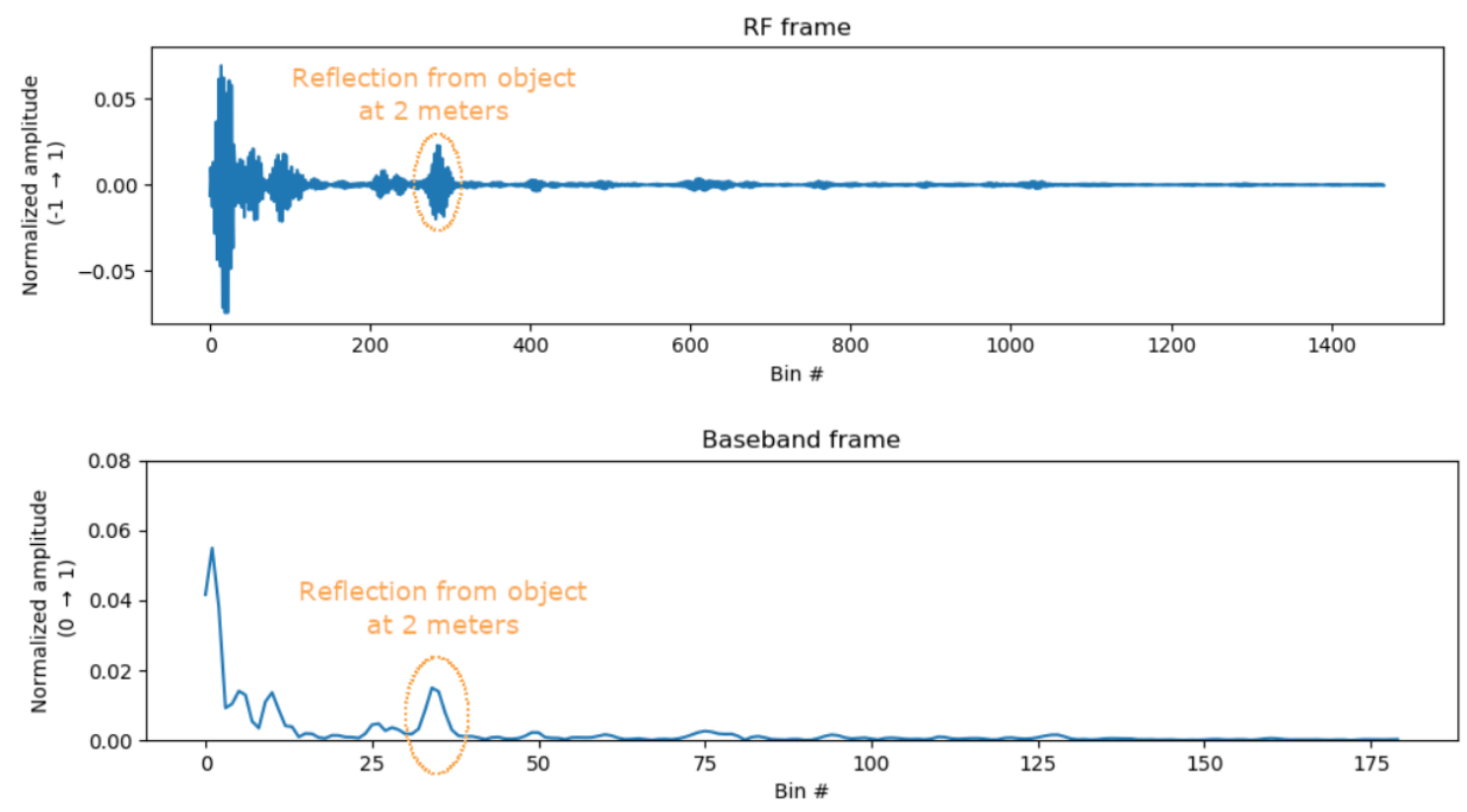

Figure 53: Radar frame and the downsampled baseband frame [50].

an effective method for occupancy sensing purposes since humans do not move very 
much. The default frames per second is only $17 \mathrm{f} / \mathrm{s}$ [51]. However, for drone microDoppler analysis, this is not sufficient since the drones can move very quickly and also have high propeller rotation speeds. The maximum frames per second is only $1.5 \mathrm{KHz}$ with this radar and this frame rate corresponds to a maximum unambiguous velocity of $15.32 \mathrm{~m} / \mathrm{s}$. Thus, this radar could only be used for HERM line analysis.

The output data of this radar is in the form of a matrix where each column represents a range bin and each row represents the radar sample in slow time.

Table 19: Xethru X4 radar specifications.

\begin{tabular}{|l|l|}
\hline Radar Specifications & \\
\hline TX center frequency & $7.29 \mathrm{GHz}$ \\
\hline Rx gain & $14.1 \mathrm{~dB}$ \\
\hline Tx bandwidth & $1.4 \mathrm{GHz}$ \\
\hline Peak Transmitter Power & $4.1 \mathrm{dBm}$ \\
\hline Rx noise figure & $6.8 \mathrm{~dB}$ \\
\hline
\end{tabular}

\section{B.2 TI AWR1642 FMCW Radar}

Texas Instrument's TI AWR1642 is an automotive FMCW radar operating in the W-band of 76- $81 \mathrm{GHz}$ and has 2 transmitters and 4 receivers for MIMO purposes. It has more configuration options than the Xethru X4 radar with a maximum chirp slope of $100 \mathrm{MHz} / \mu \mathrm{s}$ and a maximum chirp bandwidth of $4 \mathrm{GHz}$. However, it has the limitation of only being able to collect $10 \mathrm{~s}$ of data at a time.

The TI radar transmits a group of chirps called a frame. Figure 54 illustrates the chirp-frame structure.

One frame contains many chirps followed by the inter-frame time [52]. The radar data 


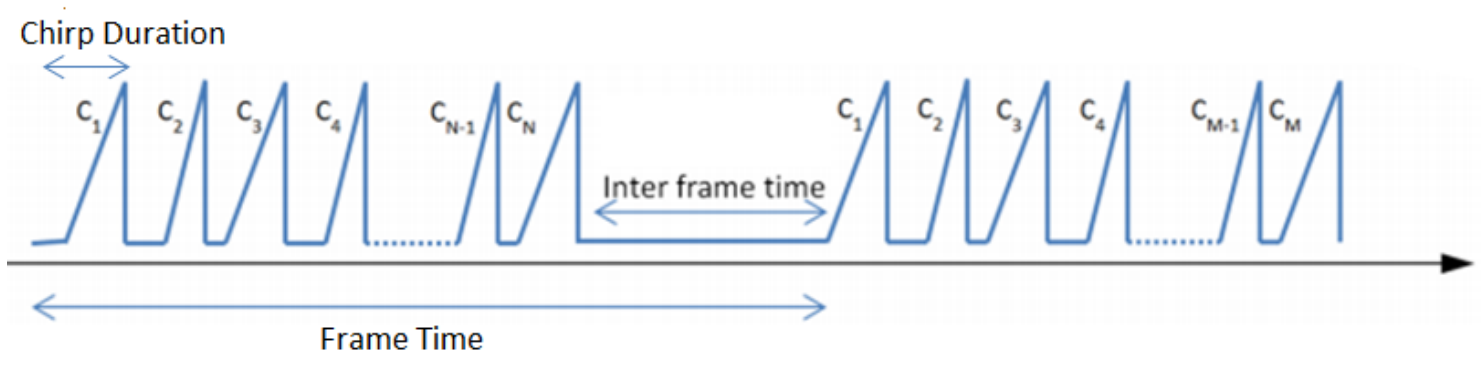

Figure 54: Chirp frame structure [52].

can then be processed on a frame-to-frame basis. The maximum number of chirps in a frame is 255. For the experiments conducted in this thesis, the inter-frame time was selected to be very short so that radar processing can be done continuously rather than on a frame-by-frame basis.

Since the Xethru X4 radar acts as a low PRF radar due to its low frame rate, the TI radar is configured to be a high PRF radar to study the blade flash phenomenon. The basic requirement of this high PRF radar is a maximum unambiguous velocity of $30 \mathrm{~m} / \mathrm{s}$. This should be enough to unambiguously sample the Doppler shift induced from the propellers of drones at idle conditions. At $77 \mathrm{GHz}$, a velocity of $30 \mathrm{~m} / \mathrm{s}$ corresponds to a Doppler shift of $15.4 \mathrm{KHz}$. This means the PRF of the radar would need to be $30.8 \mathrm{KHz}$ to satisfy the Nyquist criteria. The duration of each chirp would need to be $1 / 30.8 \mathrm{kHz}(33 \mu \mathrm{s})$. The chirp bandwidth is set to $1 \mathrm{GHz}$ so that the range resolution is $15 \mathrm{~cm}$. Under such a condition, the chirp slope is $30 \mathrm{MHz} / \mu$ s using the following equation:

$$
S=\frac{B}{T_{c}}
$$

The ADC sampling rate is $10 \mathrm{MHz}$. This results in a maximum beat frequency of $5 \mathrm{MHz}$. This means the maximum unambiguous range of this radar using this configuration is $24.75 \mathrm{~m}$ and is obtained using the following formula:

$$
R=\frac{f_{b} T_{c} c}{2 B}
$$

In order to increase this maximum range, either the chirp duration, $T_{c}$, could be 
increased or the bandwidth, $B$, could be reduced. The former would reduce the range resolution while the latter would reduce the maximum unambiguous Doppler. This demonstrates the trade-off between PRF and range.

To summarize, the chirp parameters for the TI radar are as follows.

Table 20: TI AWR1642 radar chirp parameters.

\begin{tabular}{|l|l|}
\hline Chirp slope & $30 \mathrm{MHz} / \mathrm{us}$ \\
\hline Chirp Duration & $1 \mathrm{GHz}$ \\
\hline ADC sampling rate & $10 \mathrm{MHz}$ \\
\hline Samples per chirp & 256 \\
\hline Chirp Duration & $33 \mathrm{us}$ \\
\hline Chirps per Frame & 255 \\
\hline Frame Time & $10 \mathrm{~ms}$ \\
\hline
\end{tabular}

Radar chirp configuration is done within the mmWavestudio GUI. Figure 55 shows the GUI with all the parameters that could be modified.

The radar setup consisted of 2 components, the AWR1642 radar board and the DCA1000 data capture board. They are connected together using a ribbon cable. The DCA1000 streams the radar data collected through Ethernet onto the computer. Figure 56 shows the full radar setup requiring 2 USB cables and 2 power supplies.

The recorded data is stored in a binary file and processed using a Matlab script to extract IQ data. Figure 57 shows the data matrix in Matlab. Each row corresponds to data collected from each receiver channel. MIMO is not used so the radar backscatter from all 4 receivers were summed together.

FMCW radar processing is done with a 2-D FFT. First, the data matrix is reshaped into a 256 by $n$ matrix where 256 is the number of samples per chirp (number of range bins) and $n$ is the number of chirps in slow time. A 256 sample range FFT is then computed across the samples in each chirp. A second Doppler FFT is then 


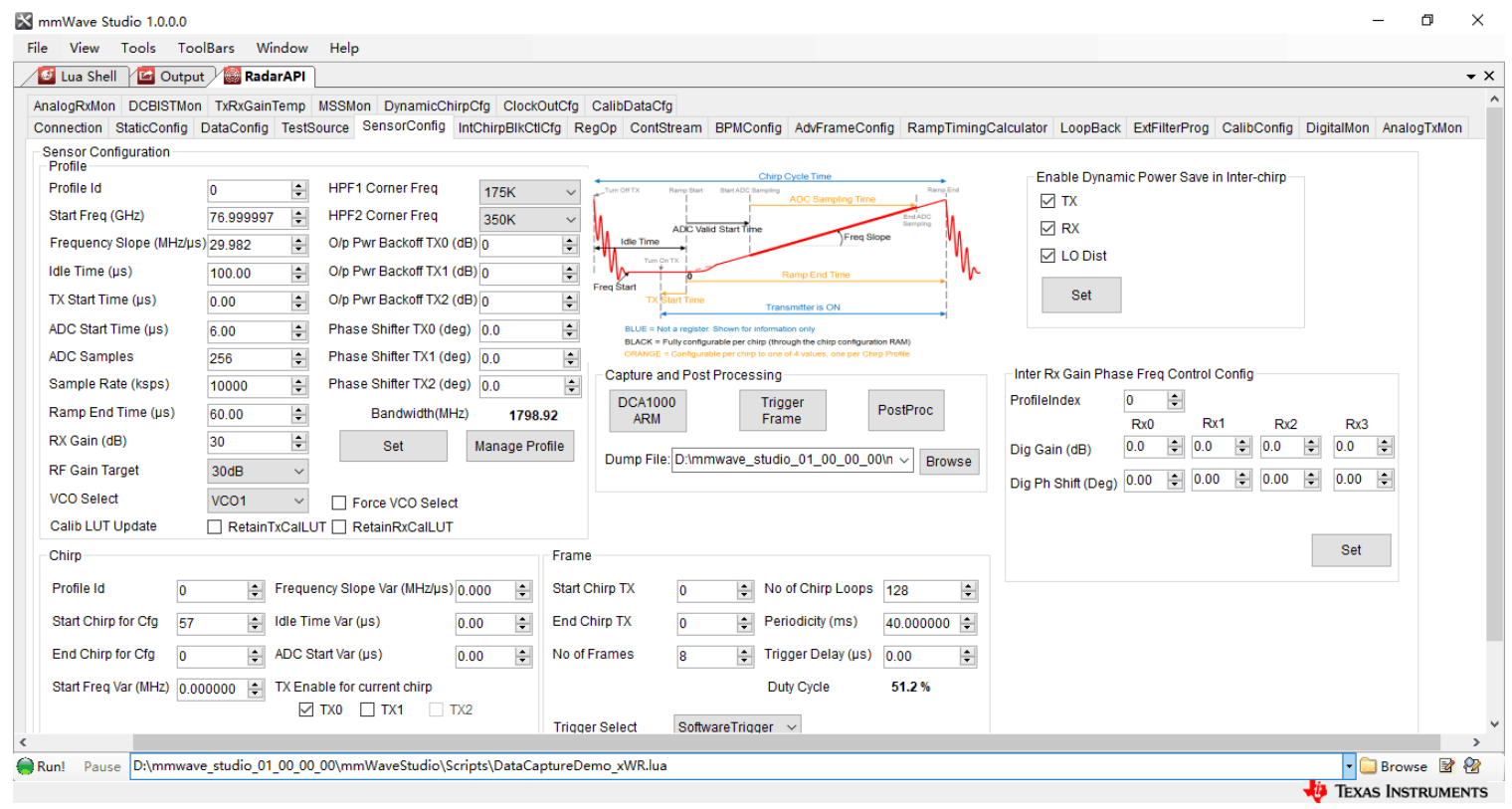

Figure 55: Sensor config in mmwave studio showing all the parameters that can be modified.

applied across the $n$ chirps to produce a range-Doppler spectrogram. Figure 58 shows an example of a range FFT and a range Doppler response of a drone fixed on a table with its rotors spinning. The main Doppler component is at DC since the drone was not moving and the sidebands represent the micro-Doppler shift from the spinning propellers. 


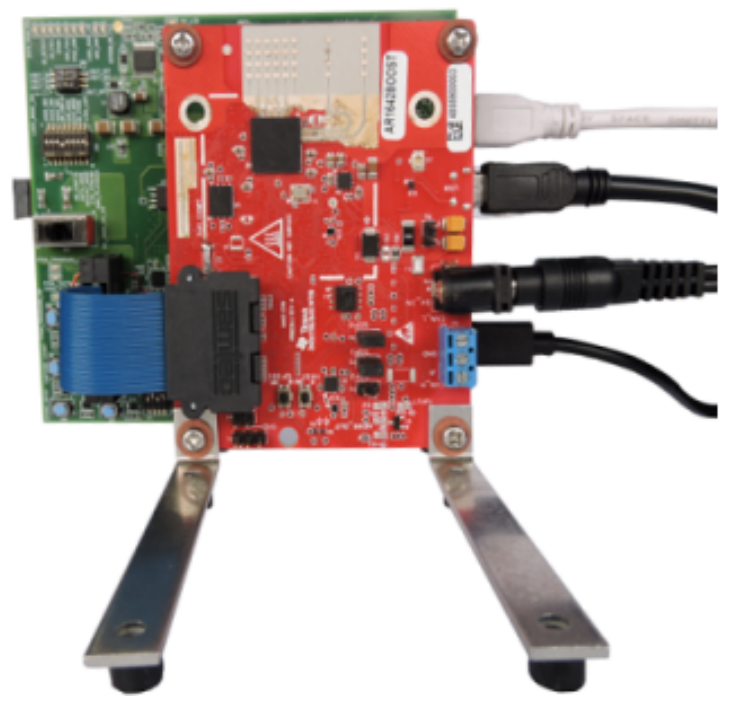

Figure 56: 2-piece radar setup. The data capture board is connected to the AWR1642 radar board using a ribbon cable.

\begin{tabular}{|c|c|c|c|c|c|c|c|c|c|c|c|c|c|c|c|c|}
\hline \multicolumn{17}{|c|}{ adcData $x$} \\
\hline \multicolumn{17}{|c|}{$\boxplus 4 \times 3276800$ complex double } \\
\hline & 1 & 2 & 3 & 4 & 5 & 6 & 7 & 8 & 9 & 10 & 11 & 12 & 13 & 14 & 15 & 1 \\
\hline 1 & $-32.0000+\ldots$ & $-47.0000-1 \ldots$ & $-43.0000-9 . .$. & $-9.0000 e+0 \ldots$ & . $4.9000 e+01 \ldots$ & $9.9000 \mathrm{e}+01 \ldots$ & . $1.7500 e+02 \ldots$ & $.2 .4700 e+02 \ldots$ & $2.5100 e+02 \ldots$ & $1.9700 e+02 \ldots$ & $.1 .1600 \mathrm{e}+02 \ldots$ & $7.5000 \mathrm{e}+01 \ldots$ & $1.0000 e+00 \ldots$ & $-4.2000 \mathrm{e}+0 \ldots$ & $-72.0000+\ldots$ & $-94.00 \wedge$ \\
\hline 2 & $-98.0000+\ldots$ & $-1.1400 \mathrm{e}+0 . .$. & $-96.0000-7 \ldots$ & $-1.5000 \mathrm{e}+0 \ldots$ & . $12.0000-97 \ldots$ & $57.0000-89 . .$. & . $1.3700 e+02 \ldots$ & $.1 .6200 e+02 \ldots$ & $1.3200 e+02 \ldots$ & . $5.4000 e+01 \ldots$ & ... $-1.4000 \mathrm{e}+0 \ldots$ & $-8.7000 e+0 \ldots$ & $-1.5500 e+0 \ldots$ & $-2.0700 \mathrm{e}+0 \ldots$ & $-2.5300 \mathrm{e}+0 .$. & -2.650 \\
\hline 3 & $15.0000-14 \ldots$ & $-32.0000-5 \ldots$ & $-9.0000 \mathrm{e}+0 . .$. & $4.4000 \mathrm{e}+01 \ldots$ & . $8.6000 e+01 \ldots$ & $1.3600 \mathrm{e}+02 \ldots$ & . $2.1900 e+02 \ldots$ & . $2.6100 e+02 \ldots$ & $2.4300 e+02 \ldots$ & $2.2000 e+02 \ldots$ & $. .2 .0700 \mathrm{e}+02 \ldots$ & $1.7300 \mathrm{e}+02 \ldots$ & $1.1100 e+02 \ldots$ & . $7.2000 \mathrm{e}+01 \ldots$ & $-1.0000+7 \ldots$ & -38.001 \\
\hline 4 & $-38.0000+\ldots$ & $-82.0000-3 . .$. & $-4.9000 \mathrm{e}+0 . \ldots$ & $2.9000 \mathrm{e}+01 \ldots$ & . 7.0000e+01 ... & $1.5700 \mathrm{e}+02 \ldots$ & $2.0500 e+02 \ldots$ & $.2 .0300 e+02 \ldots$ & $1.9700 e+02 \ldots$ & $.1 .7100 \mathrm{e}+02 \ldots$ & .. $1.1900 e+02 \ldots$ & $1.4000 \mathrm{e}+01 \ldots$ & $-5.2000 \mathrm{e}+0 \ldots$ & $-1.3700 \mathrm{e}+0 \ldots$ & $-2.0000 e+0 \ldots$ & -1.880 \\
\hline 5 & & & & & & & & & & & & & & & & \\
\hline 6 & & & & & & & & & & & & & & & & \\
\hline
\end{tabular}

Figure 57: I/Q radar data for 4 receivers. 

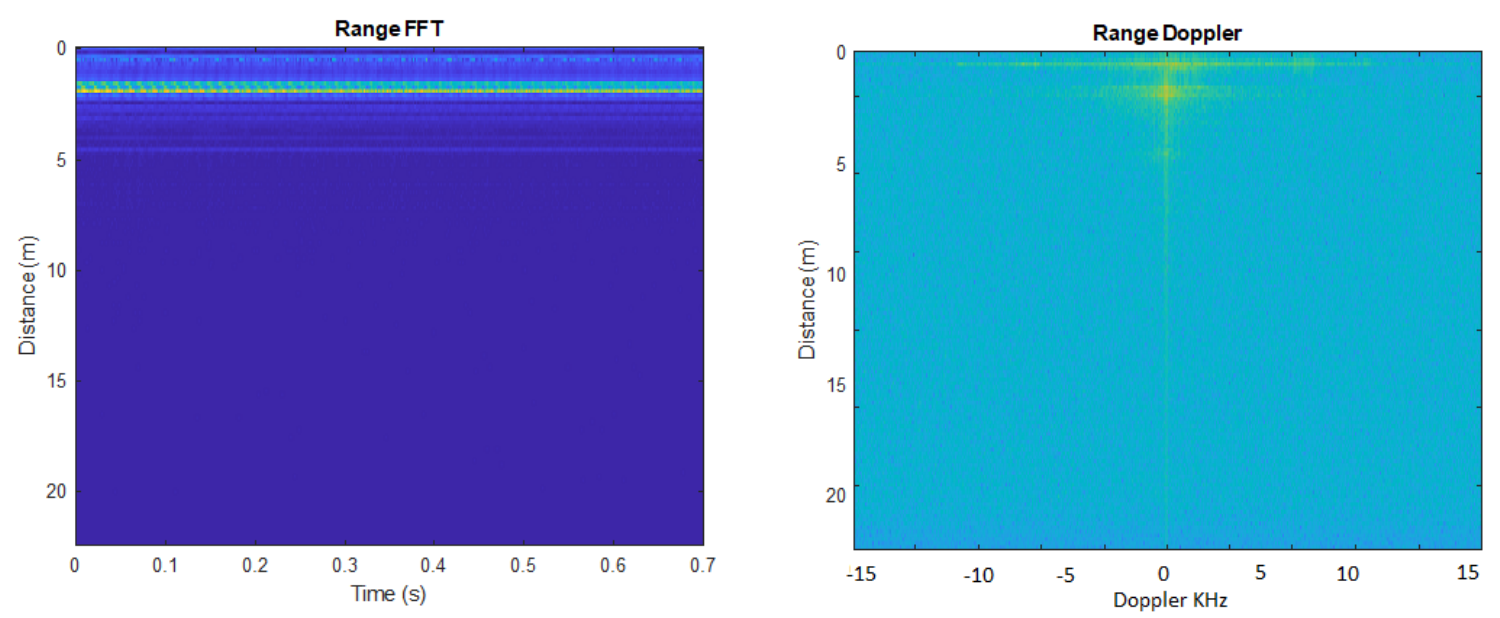

Figure 58: Range FFT spectrogram and range Doppler spectrogram.

Table 21: TI AWR1642 radar specifications.

\begin{tabular}{|l|l|}
\hline Radar Specifications & \\
\hline TX center frequency & $77-81 \mathrm{GHz}$ \\
\hline Tx gain & $10 \mathrm{~dB}$ \\
\hline Rx gain & $30 \mathrm{~dB}$ \\
\hline Max Chirp bandwidth & $4 \mathrm{GHz}$ \\
\hline Peak Transmitter Power & $12.5 \mathrm{dBm}$ \\
\hline Rx noise figure & $15 \mathrm{~dB}$ \\
\hline Max Chirp Slope & $100 \mathrm{MHz} / \mathrm{us}$ \\
\hline Max Beat Frequency & $10 \mathrm{MHz}$ \\
\hline Max range resolution & $3.75 \mathrm{~cm}$ \\
\hline
\end{tabular}




\section{B.3 Ancortek Radars}

Ancortek Inc. was a company founded by Dr. Victor C. Chen who is known for pioneering research into the micro-Doppler effect in radar [53]. They have developed many low powered software defined radars operating at S-, C-, X- and K-band. In this thesis, two of these radars were used operating in the $\mathrm{C}$ - and $\mathrm{X}$ - band. The 9.8

\begin{tabular}{|c|c|c|c|c|c|c|c|c|c|}
\hline Specifications & Min. & Typ. & Max. & Units & Specifications & Min. & Typ. & Max. & Units \\
\hline No. of $T x /$ No. of $R x$ & \multicolumn{4}{|c|}{ Single-channel Tx/Dual-channel $R x$} & No. of $T x /$ No. of $R x$ & \multicolumn{4}{|c|}{ Single-channel $\mathrm{T} x /$ Dual-channel $R x$} \\
\hline Waveforms & \multicolumn{4}{|c|}{ FMCW Sawtooth/FSK/CW } & Waveforms & \multicolumn{4}{|c|}{ FMCW Sawtooth/FSK/CW } \\
\hline Typical Frequency Limits & 9.6 & & 10 & $\mathrm{GHz}$ & Typical Frequency Limits & 5.6 & & 6.0 & $\mathrm{GHz}$ \\
\hline Typical Bandwidth & 0 & & 400 & $\mathrm{MHz}$ & Typical Bandwidth & 0 & & 400 & $\mathrm{MHz}$ \\
\hline Expandable Frequency Limits & 9 & & 10 & $\mathrm{GHz}$ & Expandable Frequency Limits & 5.2 & & 6.0 & $\mathrm{GHz}$ \\
\hline Expandable Bandwidth & 0 & & 1 & $\mathrm{GHz}$ & Expandable Bandwidth & 0 & & 800 & $\mathrm{MHz}$ \\
\hline FMCW Sweep Time & \multicolumn{4}{|c|}{$0.125 / 0.25 / 0.5 / 1 / 2 / 4 / 8(\mathrm{~ms})$} & FMCW Sweep Time & \multicolumn{4}{|c|}{$0.125 / 0.25 / 0.5 / 1 / 2 / 4 / 8(\mathrm{~ms})$} \\
\hline Number of Samples/Sweep & \multicolumn{4}{|c|}{$8 / 16 / 32 / 64 / 128 / 256 / 512 / 1024 / 2048 / 4096$} & Number of Samples/Sweep & \multicolumn{4}{|c|}{$8 / 16 / 32 / 64 / 128 / 256 / 512 / 1024 / 2048 / 4096$} \\
\hline Tuning Voltage & 0 & & 5 & $\mathrm{v}$ & Tuning Voltage & 0 & & 5 & $\mathrm{v}$ \\
\hline Tuning Sensitivity @RF Port & & 0.4 & & $\mathrm{GHz} / \mathrm{N}$ & Tuning Sensitivity @RF Port & & 0.13 & & $\mathrm{GHz} / \mathrm{N}$ \\
\hline Transmit Power & 17 & 19 & 21 & $\mathrm{dBm}$ & Transmit Power & 20 & 21 & 22 & $\mathrm{dBm}$ \\
\hline SSB Phase Noise @1MHz offset & & -109 & & $\mathrm{dBC} / \mathrm{Hz}_{2}$ & SSB Phase Noise @1MHz offset & & -137 & & $\mathrm{dBc} / \mathrm{H}_{2}$ \\
\hline Noise Figure & & 1.8 & & $d B$ & Noise Figure & & 1.8 & & $d B$ \\
\hline Maximum input power & & 22 & & $\mathrm{dBm}$ & Maximum input power & & 10 & & $d B m$ \\
\hline Supply voltage & 4.75 & 5 & 5.25 & $\mathrm{v}$ & Supply voltage & 4.75 & 5 & 5.25 & v \\
\hline Supply current & 1980 & 2030 & & $\mathrm{~mA}$ & Supply current & 1040 & 1070 & & $\mathrm{~mA}$ \\
\hline Operating temperature & -40 & & 85 & $c^{0}$ & Operating temperature & -40 & & 85 & $c^{0}$ \\
\hline Dimensions & \multicolumn{3}{|c|}{$L=138 \mathrm{~W}=103 \quad \mathrm{H}=30$} & $\mathrm{~mm}$ & Dimensions & \multicolumn{3}{|c|}{$L=138 \mathrm{~W}=103 \mathrm{H}=30$} & $\mathrm{~mm}$ \\
\hline
\end{tabular}

Figure 59: Left: Ancortek SDR980AD2 X-Band radar specifications. Right: Ancortek SDR580 AD2 C-Band radar specifications.

GHz radar is stated to be able to detect a target of RCS $1 \mathrm{sm}$ up to $80 \mathrm{~m}$ at a SNR of $13 \mathrm{~dB}$ while the $5.8 \mathrm{GHz}$ can detect a target of RCS $1 \mathrm{sm}$ up to $120 \mathrm{~m}$ at a SNR of 13 dB. These radars were used in the FMCW mode. Unlike the TI AWR1642 radar, the chirps are not transmitted in the chirp frame structure and they do not have MIMO capability. Figure 60 shows the experimental setup of the two radar systems. Both radars were running side by side and at the same time. 


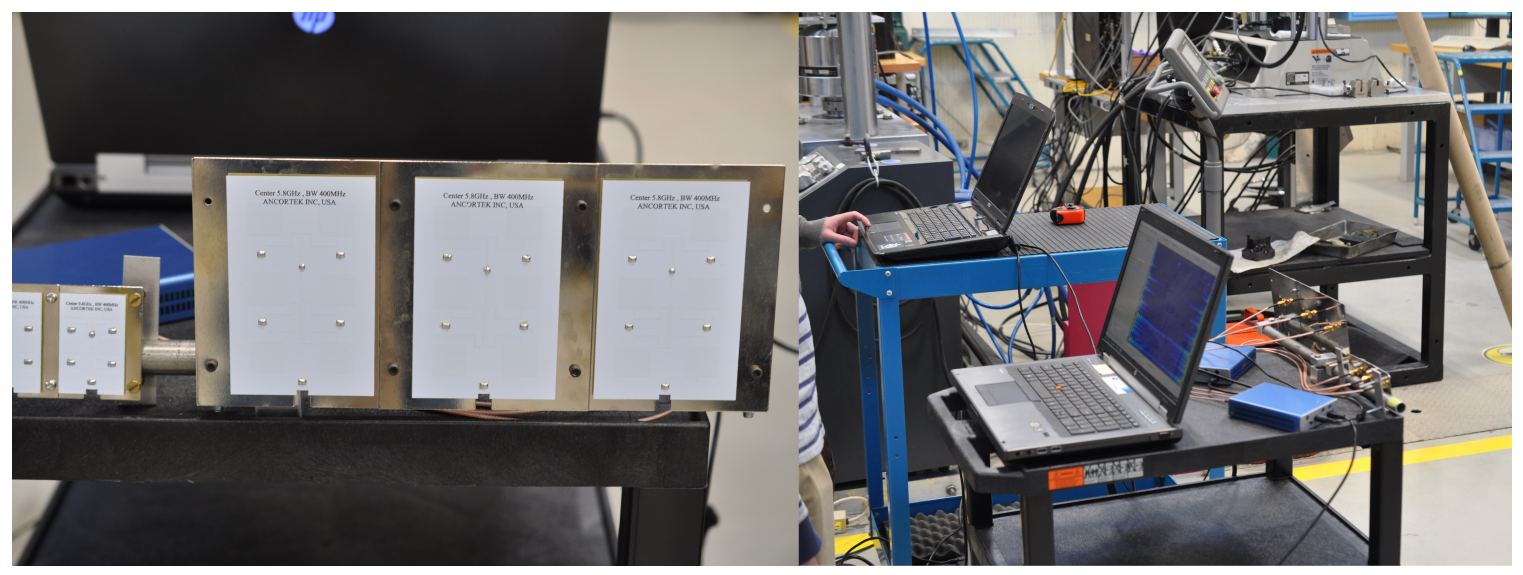

Figure 60: Radar setup for the Ancortek Radars. Both radars were operating at the same time. 


\section{Appendix C}

\section{Radar Performance Evaluation}

This section provides an estimate of the minimum radar input SNR required for the HERM line signal processing outlined in this thesis. It is known that micro-Doppler based techniques are only viable for short ranges due to the small rotors but this has never been quantified in terms of the radar specifications.

This minimum SNR for micro-Doppler analysis was estimated using experiments conducted with two Ancortek radars, the SDR-KIT 980 AD2 and the SDR-KIT 580 AD2. The two radars have similar specs except for the different carrier frequency and transmit power. This allows for a comparison of how different frequency bands affect micro-Doppler acquisition. The specifications of these 2 radars are shown in Table 22 .

Range experiments were conducted by fixing the DJI Spark at varying distances up to $10 \mathrm{~m}$ in line of sight with the radar. Both radars were operating at the same time. The maximum range at which HERM lines were visible was at $10 \mathrm{~m}$ for the 5.8 $\mathrm{GHz}$ radar and $6 \mathrm{~m}$ for the $9.8 \mathrm{GHz}$ radar. This is expected since the $5.8 \mathrm{GHz}$ radar has more transmit power and longer wavelength. The STFT spectrograms are shown 
Table 22: Ancortek radar specifications.

\begin{tabular}{|l|l|l|}
\cline { 2 - 3 } \multicolumn{1}{c|}{} & SDR-KIT 580 & SDR-KIT 980 \\
\hline Pt & $0.1 \mathrm{~W}$ & $0.08 \mathrm{~W}$ \\
\hline PRF & $2 \mathrm{kHz}$ & $2 \mathrm{kHz}$ \\
\hline Fc & $5.8 \mathrm{GHz}$ & $9.8 \mathrm{GHz}$ \\
\hline Gain & $14 \mathrm{~dB}$ & $14 \mathrm{~dB}$ \\
\hline Chirp Bandwidth & $400 \mathrm{MHz}$ & $400 \mathrm{MHz}$ \\
\hline Noise Figure & $3.4 \mathrm{~dB}$ & $3.4 \mathrm{~dB}$ \\
\hline IF Bandwidth & $4 \mathrm{MHz}$ & $4 \mathrm{MHz}$ \\
\hline
\end{tabular}

below in 61 for the two ranges generated using a window size of 256 .
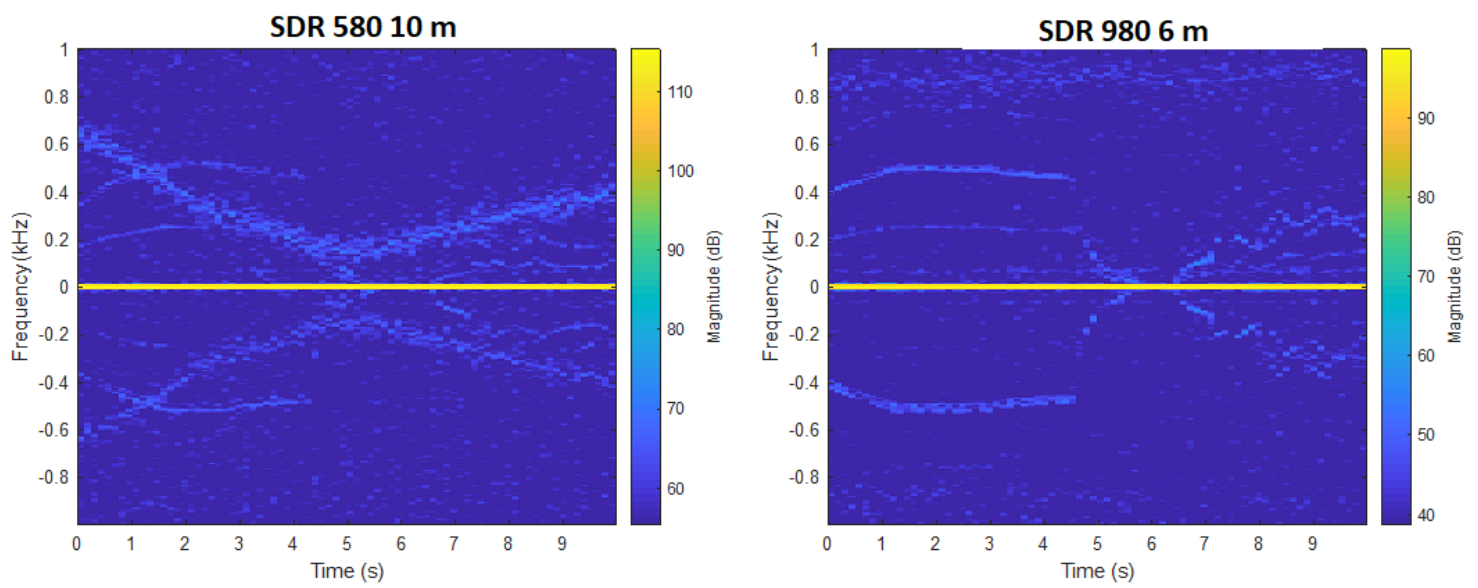

Figure 61: HERM line spectrograms for DJI Spark.

At this range, the HERM lines start to fade leaving only the HERM line components corresponding to the maximum micro-Doppler shift (blade tip). This is a factor to consider when developing long range STFT based drone classification techniques.

Given these ranges and the specifications of the radars, the radar range equation was used to estimate the input SNR. The RCS of the DJI Spark is approximated to be $0.001 \mathrm{~m}^{2}$ since it is smaller than a DJI Phantom which is known to have an RCS of 
$0.01 m^{2}[54]$. The calculated input SNR is tabulated in the table below.

Table 23: Input SNR Calculation.

\begin{tabular}{|l|l|l|}
\hline & $5.8 \mathrm{GHz}$ & $9.8 \mathrm{GHz}$ \\
\hline $\mathrm{RCS}=0.001 m^{2}$ & $23.56 \mathrm{~dB}$ & $27.03 \mathrm{~dB}$ \\
\hline
\end{tabular}

The input SNR is $23.56 \mathrm{~dB}$ for the $5.8 \mathrm{GHz}$ radar $27.03 \mathrm{~dB}$ for the $9.8 \mathrm{GHz}$. This makes sense since the SNR of the HERM lines for the $9.8 \mathrm{GHz}$ radar is slightly higher in Figure 61. When a minimum input SNR of $23.56 \mathrm{~dB}$ is used for the $9.8 \mathrm{GHz}$ radar, the maximum range is calculated to be $7.3 \mathrm{~m}$. Figure 62 shows the HERM line signature of the DJI Spark at $8 \mathrm{~m}$ captured using the $9.8 \mathrm{GHz}$ radar. This signature is much weaker so an estimated maximum range of $7.3 \mathrm{~m}$ makes sense. Thus, a

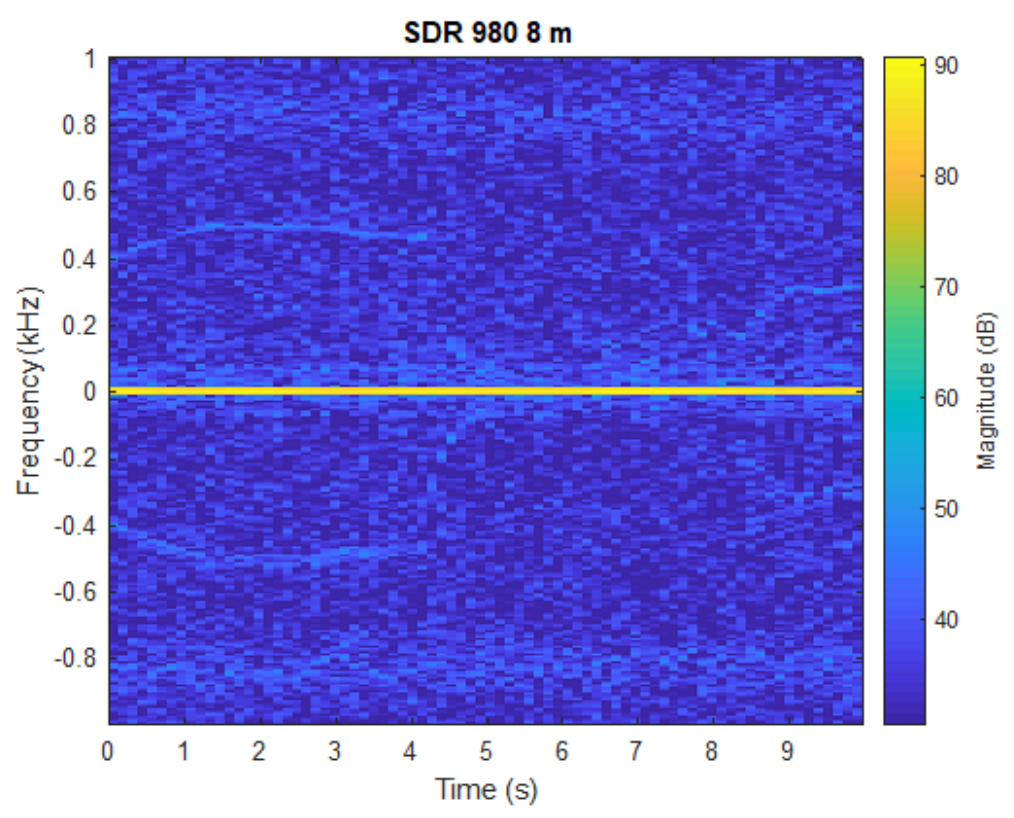

Figure 62: HERM line spectrogram for DJI Spark at $8 \mathrm{~m}$ captured using the $9.8 \mathrm{GHz}$ radar.

minimum SNR of $23.56 \mathrm{~dB}$ works well at estimating the maximum range for microDoppler analysis using both C- and X-band radar. This can be used to estimate the maximum range for micro-Doppler analysis for other drones such as the DJI Phantom 
$\left(R C S=0.01 \mathrm{~m}^{2}\right)$ and for other $\mathrm{C}$ and $\mathrm{X}$-band radar systems. For reference, detecting a Swerling I target with a $P_{d}$ of 0.93 and a $P_{f a}$ of $10^{-6}$ is about $23 \mathrm{~dB}$. Multi-rotor drones can be represented as Swerling I targets since they are slow moving and each spinning rotor counts as an independent scatterer [54]. 